\title{
Role of transthoracic impedance on the success of electrical synchronized cardioversion
}

\author{
By \\ Varsha Chaugai, B-Tech
}

A thesis submitted to the Faculty of Graduate Studies and Research in partial fulfillment of the requirements for the degree of
Masters of Applied Science
in Biomedical Engineering

Ottawa-Carleton Institute for Biomedical Engineering

Department of Systems and Computer Engineering

Carleton University

Ottawa, Ontario, Canada

August 2012

Copyright (c) Varsha Chaugai, 2012 
Library and Archives

Canada

Published Heritage

Branch

395 Wellington Street

Ottawa ON K1A ON4

Canada
Bibliothèque et

Archives Canada

Direction du

Patrimoine de l'édition

395 , rue Wellington

Ottawa ON K1A ON4

Canada
Your file Votre référence

ISBN: 978-0-494-93485-2

Our file Notre référence

ISBN: 978-0-494-93485-2
NOTICE:

The author has granted a nonexclusive license allowing Library and Archives Canada to reproduce, publish, archive, preserve, conserve, communicate to the public by telecommunication or on the Internet, loan, distrbute and sell theses worldwide, for commercial or noncommercial purposes, in microform, paper, electronic and/or any other formats.

The author retains copyright ownership and moral rights in this thesis. Neither the thesis nor substantial extracts from it may be printed or otherwise reproduced without the author's permission.
AVIS:

L'auteur a accordé une licence non exclusive permettant à la Bibliothèque et Archives Canada de reproduire, publier, archiver, sauvegarder, conserver, transmettre au public par télécommunication ou par l'Internet, prêter, distribuer et vendre des thèses partout dans le monde, à des fins commerciales ou autres, sur support microforme, papier, électronique et/ou autres formats.

L'auteur conserve la propriété du droit d'auteur et des droits moraux qui protege cette thèse. $\mathrm{Ni}$ la thèse ni des extraits substantiels de celle-ci ne doivent être imprimés ou autrement reproduits sans son autorisation.
In compliance with the Canadian Privacy Act some supporting forms may have been removed from this thesis.

While these forms may be included in the document page count, their removal does not represent any loss of content from the thesis.
Conformément à la loi canadienne sur la protection de la vie privée, quelques formulaires secondaires ont été enlevés de cette thèse.

Bien que ces formulaires aient inclus dans la pagination, il n'y aura aucun contenu manquant. 


\section{Abstract}

The thesis examines the effect of transthoracic impedance (TTI) on the success rate of synchronized cardioversion, using retrospective analysis on clinical data and numerical model. Synchronized electrical cardioversion is a treatment that applies an electrical pulse to the cardiac tissue to restore normal sinus rhythm in patients presenting atrial fibrillation (AF), atrial flutter (AFL) and ventricular tachycardia (VT). The success of this treatment is largely dependent on the current density at the heart, which is inversely related to $\pi I$. Hence, during cardioversion it is desirable to have lower TTI and deliver an "optimal" current based on the patient's impedance; that is, the minimum current density that provides a successful cardioversion. Relative to a monophasic electric pulse, the "impedance compensating" biphasic pulse used in the defibrillation technology today is understood to compensate for variation in impedance. While the biphasic technology efficiently compensates for the variation in impedance, the extent of its influence on positive outcomes for patients exhibiting high TTI is still unclear.

This thesis examines the effect of TTI on the success of cardioversion in two parts. The first part involves retrospective analysis on the clinical data obtained from University of Ottawa Heart Institute. The second part develops a 3-D model of the human thorax using Finite Element Method (FEM) to simulate the current density distribution for subjects with high, average and low TTI. This work also investigates the effect of pad placement (e.g., anterior-lateral and anterior-posterior positions) that can better inform clinicians when developing cardioversion procedures.

Results from retrospective study and the FEM validate our current understanding on the influence of TTI on the success rate. It was also found that one of the ways to improve the efficacy of cardioversion would be to defibrillate the patients with pads on the anterior-posterior position. 


\section{Acknowledgements}

I am deeply indebted to my supervisors Dr. Andy Adler, Dr. Adrian D.C. Chan, and Mr. Timothy Zakutney for providing me with an opportunity to pursue my Master's degree at Carleton University. This thesis would not have been possible without their help, advice, and encouragement in all the time of research for and writing of this thesis.

My sincere thanks go to Dr. Mouhannad Sadek and Dr. David Birnie for giving their valuable inputs on the clinical defibrillation data. I would like to thank Mark Cleland, Joshua Henne and all my colleagues at University of Ottawa Heart Institute and Carleton University for helping me progress through my masters. I am truly grateful to Dave Dawson and Raza Bhatia for their motivating words and proof reading my work.

I am eternally obliged to my friends- Anne Claude Schellenberg, Duncan Keith, Robert Pushman, Gurwinder Khabra, Glaiza Ponce, Andy Huang and Graham Fraser. Their friendship, patience, humor, and motivation have made my two years stay in Ottawa one of the best experiences I have had.

Above all, I would like to thank my parents, brother and my entire family in Nepal for their love and unequivocal support, for which my mere expression of thanks does not suffice.

I am grateful to the university and department of Systems and Computer Engineering, MITACS, and Carleton Innovative Fund for providing me with financial support. 
To my family,

for being a great source of motivation and inspiration. 


\section{Table of Contents}

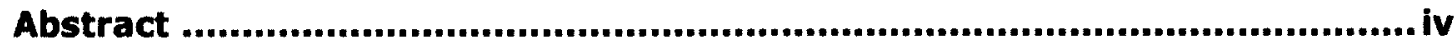

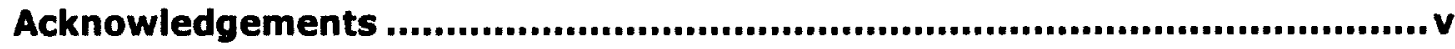

Table of Contents ............................................................................... vii

List of Tables ..................................................................................... ix

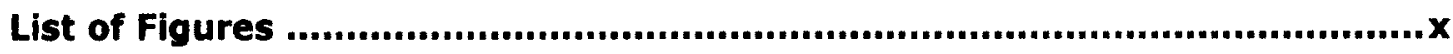

List of Symbols ................................................................................... xii

List of Abbreviations .............................................................................. xiii

1 Introduction ..................................................................................... 14

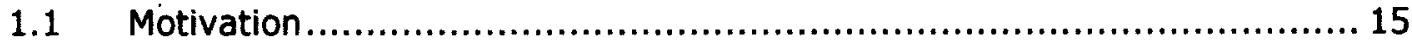

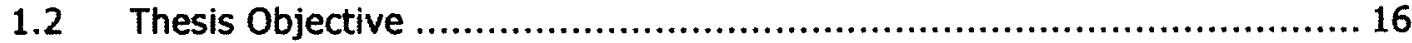

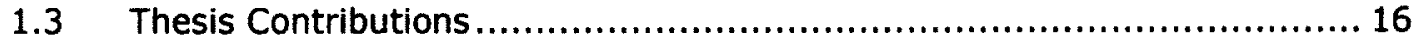

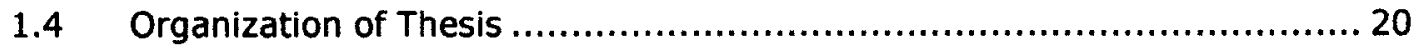

2 Literature Review ............................................................................. 21

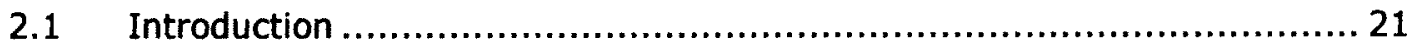

2.2 Conduction System of the heart ................................................ 21

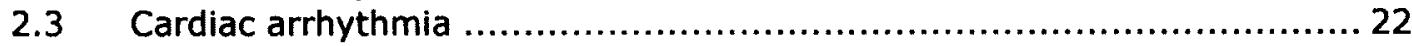

2.3.1 Types of Cardiac Arrhythmia ........................................... 22

2.3.2 Physiology of Cardiac Arrhythmia .......................................... 23

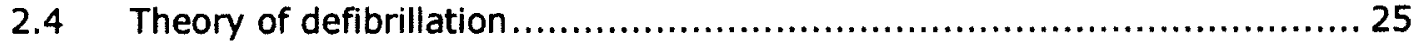

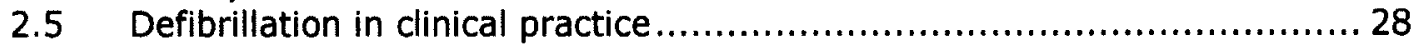

2.6 Defibrillation technology ........................................................ 28

2.6.1 Defibrillator waveforms ................................................. 29

2.7 Effect of transthoracic impedance ................................................ 33

2.7.1 Impedance compensation ............................................... 37

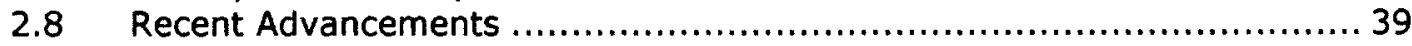

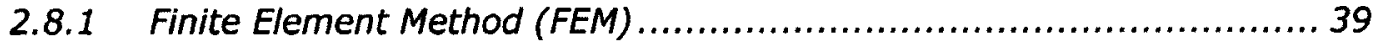

2.8.2 Triphasic waveforms................................................... 40

2.8.3 Alternating Current $(A C)$ defibrillation ................................. 40

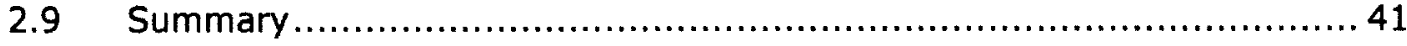

3 Experimental Methods ................................................................ 43

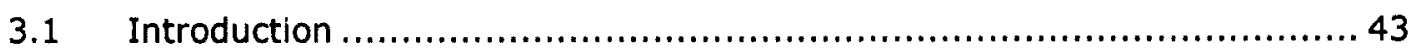

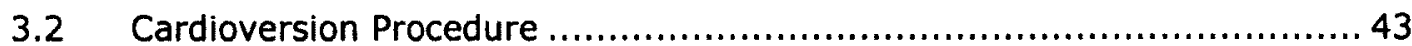

3.3 Data Acquisition.................................................................. 44

3.4 Data Processing .................................................................. 45

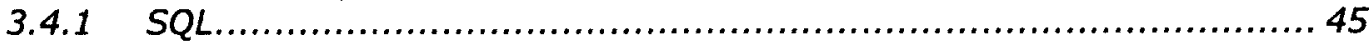

3.4.2 ECG Analysis ............................................................. 45

3.5 Measurement of voltage and waveform .................................... 46

3.6 Curve Fitting ...................................................................... 50

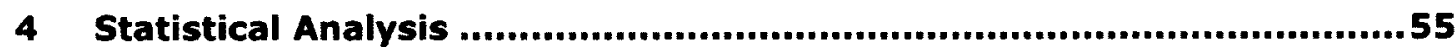

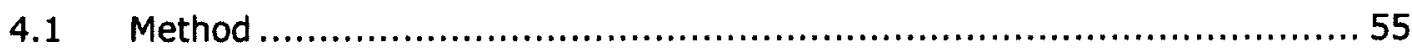

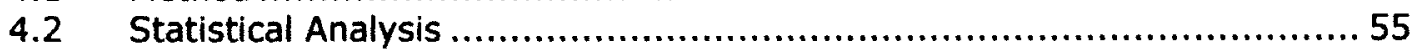

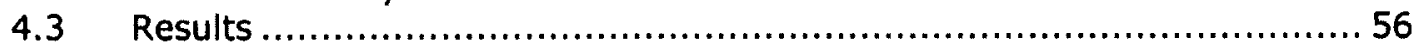

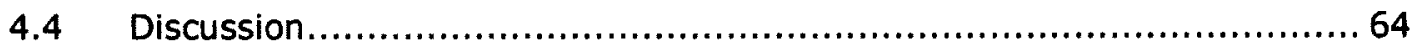




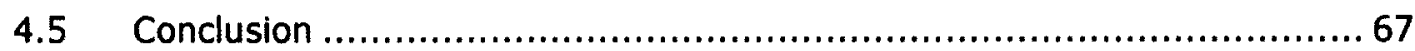

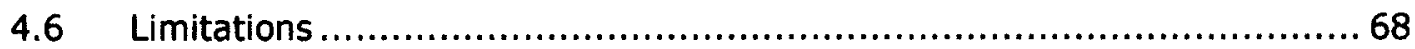

5 Finite Element Method ..........................................................................70

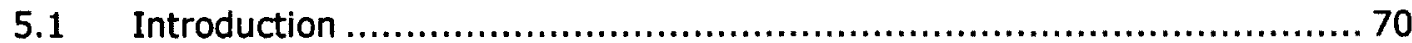

5.2 Problem Formulation ............................................................. 71

5.3 Computational Modeling using FEM ............................................ 73

$5.4 \quad$ Numerical Methods................................................................ 75

5.5 Imposing Boundary Condition .................................................... 77

5.6 Derivation of Linear Interpolation function ' $\phi$ '................................. 78

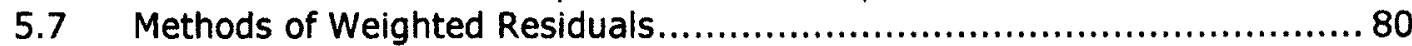

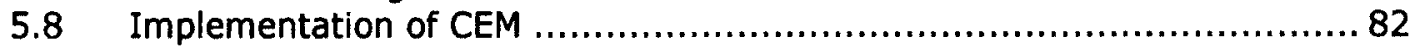

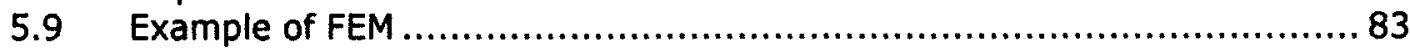

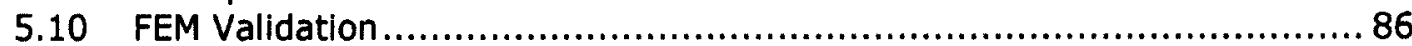

6 Finite Element Method Results ..............................................................88

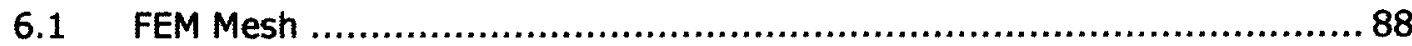

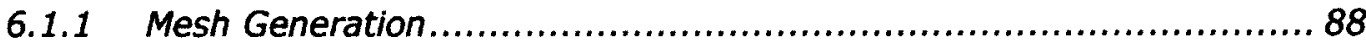

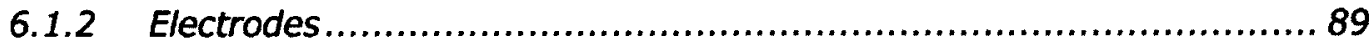

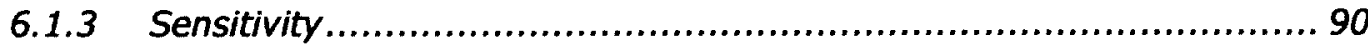

6.2 Computation of Defibrillation Parameters ....................................... 91

6.3 Effect of different patient size and impedance on the current distribution in

the heart ........................................................................................ 94

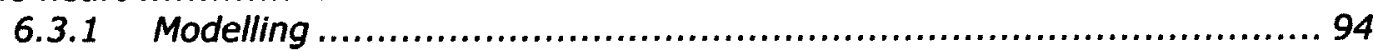

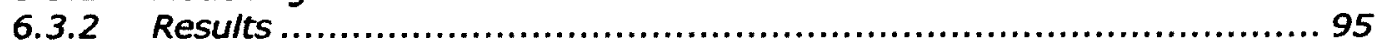

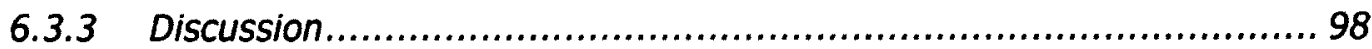

6.3.4 Conclusion …..................................................................... 100

6.4 Effect of pad positions ........................................................... 101

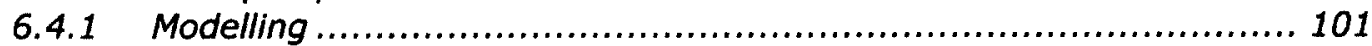

6.4.2 Statistical Analysis ............................................................ 103

6.4 .3 Results of pad position.................................................... 103

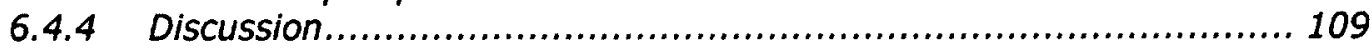

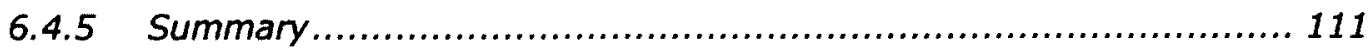

6.5 Pad position and sizes ......................................................... 112

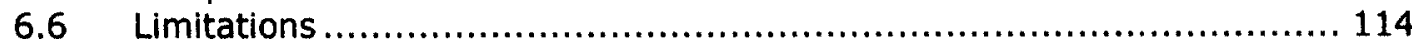

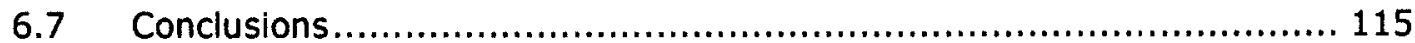

7 Thesis Summary and Future Recommendations ................................116

7.1 Summary of conclusions ......................................................... 116

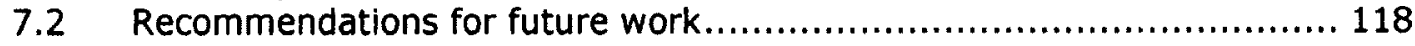

Appendix I: CLINICAL DATA ...................................................................123

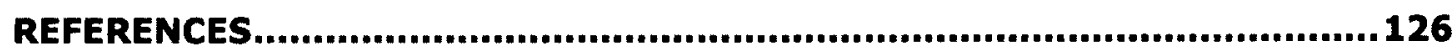




\section{List of Tables}

Table 2.1 Comparison of Monophasic waveform (MW) over Biphasic waveform (BW) for cardioversion ................................................................. 31

Table 3.1 Resistance (R), Current (I), Voltage (V) and Duration ( $t$ ) of the biphasic pulse for given energy and resistance values for HeartStart XL defibrillator..... 48

Table 3.2 Values of the coefficients of $y=a x b+c$ for 100,150 and $200 \mathrm{~J}$.............54

Table 4.1: Effect of transthoracic impedance on success rates of shocks: All shocks 57

Table 4.2: Effect of transthoracic impedance on success rates of shocks: First shock

Table 4.3 : Effect of energy of the success rates for different levels of impedance: All shocks ....................................................................................... 59

Table 6.1 Conductivity values reported for lungs and heart........................... 90

Table 6.2 FEM results for Mean, maximum and minimum conductivity for lungs and heart................................................................................. 90

Table 6.3 Difference in the defibrillation parameters in larger person with and without fat .98

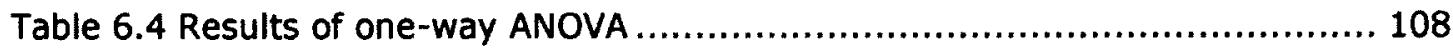

Table 6.5 Results of Tukey's HSD test ............................................. 109 


\section{List of Figures}

Figure 2.1 Electrical Conduction System of the Heart. Credit: Patrick J. Lynch, medical illustrator; C. Carl Jaffe, MD, cardiologist. http://creativecommons.org /licenses/by/2.5 ..................................................................... 21

Figure 2.2 Vulnerable period during sinus rhythm ..................................... 24

Figure 2.3 Re-entrant circuit as a result of long pathway. Based on [14]............ 25

Figure 2.4 Circuit diagram of a DC Defibrillator. Based on http://coep.vlab.co.in .... 29

Figure 2.5: Defibrillator Waveforms .................................................. 30

Figure 2.6 Standard Paddle placement for cardioversion................................ 36

Figure 2.7 Impedance Compensation by HeartStart Philips MRX for energy at $150 \mathrm{~J}$. Reproduced from [9]................................................................... 38

Figure 2.8 Triphasic waveforms used for defibrillation ............................... 40

Figure 3.1 Experimental setup for measuring peak voltage. ........................ 47

Figure 3.2 Exponential biphasic waveform for (a) $150 \mathrm{~J}, 100 \Omega$ (b) $150 \mathrm{~J}, 150 \Omega \ldots 48$

Figure 3.3 Comparison of current recorded from MRX oscilloscope and MRX device. 49

Figure 3.4 Curve fitting with exponential function $y=a e^{b x}$ for HeartStart $\mathrm{XL}$ defibrillator ............................................................................ 51

Figure 3.5 Curve fitting for power function $y=a x^{b}$ for HeartStart XL defibrillator .... 52 Figure 3.6 Curve fitting for power function $y=a x^{b}+c$ for HeartStart $X L$ defibrillator 52 Figure 4.1 Success rate of AF, AFL, and VT for low $(<=70 \Omega)$ and high impedance $(>70 \Omega)$ : All shocks. .................................................................. 57

Figure 4.2 First Shock Success rate of AF, AFL, and VT for low $(<=70 \Omega$ ) and high impedance $(>70 \Omega)$ : First Shock...................................................59

Figure 4.3 Success rates of AF, AFL, and VT for energy $>150 \mathrm{~J}$ and $200 \mathrm{~J}$ : All shocks

Figure 4.4 Relationship between Defibrillation success and Impedance for a. AF, b. AFL, and $c$. VT. ' $n$ ' represents number of shocks in a given impedance range. . 61

Figure 4.5 Relationship between peak current and shock success for a. AF, b. AFL, and $c$. VT ........................................................................... 63

Figure 4.6 Relationship between voltage and duration of the waveform for $200 \mathrm{~J}$ energy .................................................................................. 65

Figure 5.1 Example of FEM discretization a) 2-D mesh with 467 elements and b) 3-D mesh with 37874 elements ........................................................... 74

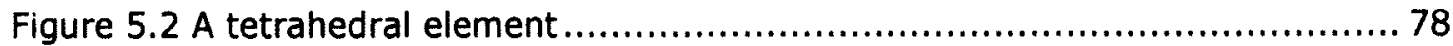

Figure 5.3 2-D FEM with 3 elements. Green circles represent point electrodes....... 83

Figure 5.4 3-D FEM cylindrical model with two electrodes (in green) enclosing a smaller cylindrical object ............................................................ 86

Figure 5.5 Electrical representation of the model ...................................... 86

Figure 6.1 Summary of the FEM mesh generation process ........................... 88

Figure 6.2 Kendall Medi-Trace $1710 \mathrm{H}$ Defibrillation Electrodes used during defibrillation........................................................................... 89

Figure 6.3 Conductivity of different tissue regions ................................... 91

Figure 6.4 Thorax Model showing only the heart. Elements colored in maroon represents the heart region and current density is calculated in those blue elements. ........................................................................... 92

Figure 6.5 Calculation of Heterogeneity Index of the mean current density in the heart.................................................................................. 93

Figure 6.6 FEM Models of a i. thin, ii. normal, and iii. larger patients. $a$. View from above $b$. View from front. Colours blue represent lungs, maroon - heart, greenelectrodes, and white- soft tissues................................................ 94 
Figure 6.7 Voltage, and Current streamlines and distribution in a central slice for $a$. Thin, $b$. Normal, and $c$. Large sized person ....................................... 96

Figure 6.8 Mean Current Density for thin, normal, and large person for 25A input current........................................................................... 97

Figure 6.9 Minimum current and energy requirement for thin, normal, and large person. No cardiac damage was seen for all cases. ................................ 97

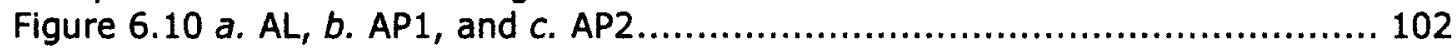

Figure 6.11 3-D Human Thorax Model in AL position: a. Anterior view, b. Posterior Side (Right). Dark Blue represents Ribs and spine, Red-Heart and Blue- Lungs

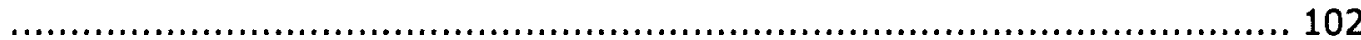

Figure 6.12 Shifts in the electrode positions........................................... 103

Figure 6.13 Voltage and Current distribution for a. AL (Top), b. AP1 (Middle), and c. AP2 position (Bottom) .............................................................. 104

Figure 6.14 Mean Current Density in heart for AL, AP1, and AP2 positions. The 25 bars in each positions shows the mean current density when the location is changed to $20 \mathrm{~mm}$ left, right, up and bottom.................................. 105

Figure 6.15 Box plot showing variation in Heterogeneity Index (HI)between the three positions with their shifts in the locations ....................................... 106

Figure 6.16 Variation in current requirement within and between the three positions ...................................................................................... 106

Figure 6.17 Comparison of pad positions with respect to resistance ................... 107

Figure 6.18 Correlation between Heterogeneity Index and Energy ................... 108

Figure 6.19 Mean Current Density for different sizes and positions .................. 113

Figure 6.20 Current and energy requirement for different pad position and sizes.. 114

Figure 7.1 Calculation of current in an object. Blue represents a spherical ball and black is a boundary element. ...................................................... 121 


\section{List of Symbols}

\begin{tabular}{|c|c|}
\hline$A$ & Area \\
\hline $\boldsymbol{w}$ & Coefficients that weigh the interpolation function \\
\hline$\sigma$ & Conductivity \\
\hline $\boldsymbol{C}$ & Connectivity Matrix \\
\hline$z_{c}$ & Contact Impedance \\
\hline$\vec{\jmath}$ & Current Density \\
\hline $\boldsymbol{I}_{\boldsymbol{l}}$ & Current in the $t^{\text {th }}$ electrode \\
\hline$N_{D}$ & Dimension of the model \\
\hline$\vec{E}$ & Electric Field \\
\hline $\boldsymbol{l}$ & Electrode I \\
\hline $\boldsymbol{U}_{\boldsymbol{l}}$ & Electrode Potential \\
\hline$\phi$ & Linear Interpolation Function \\
\hline $\boldsymbol{S}_{\boldsymbol{e}}$ & Local matrix of each element \\
\hline $\overrightarrow{\boldsymbol{B}}$ & Magnetic Field Density \\
\hline$\Gamma$ & Medium Boundary \\
\hline $\boldsymbol{\Omega}$ & Medium Space \\
\hline$J_{t h}$ & Minimum current density required for successful defibrillation \\
\hline$N_{n}$ & Number of nodes \\
\hline$N_{e}$ & Number of tetrahedral elements \\
\hline $\boldsymbol{U}$ & Potential in the element \\
\hline $\boldsymbol{R}$ & Resistance \\
\hline $\boldsymbol{u}$ & Scalar Potential \\
\hline $\boldsymbol{Y}$ & System Matrix \\
\hline$\widehat{\boldsymbol{n}}$ & Unit vector, outward normal from the boundary $d \Gamma$ \\
\hline$Q$ & Vector of Input Current \\
\hline $\boldsymbol{V}$ & Vector of Nodal Potentials \\
\hline$E_{l}$ & Voltage on the electrode 1 \\
\hline $\boldsymbol{v}$ & Weighted Function \\
\hline
\end{tabular}




\section{List of Abbreviations}

\begin{tabular}{|c|l|}
\hline 2-D & 2-Dimensional \\
\hline 3-D & 3-Dimensional \\
\hline AC & Alternating Current \\
\hline AF & Atrial Fibrillation \\
\hline AFL & Atrial Flutter \\
\hline AL & Antero-Lateral \\
\hline ANOVA & Analysis of Variance \\
\hline AP1 & Antero-Posterior1 \\
\hline AP2 & Antero-Posterior2 \\
\hline AV & Atrio-Ventricular \\
\hline BEM & Boundary Element Method \\
\hline CEM & Complete Electrode Model \\
\hline CAT & Computerized Axial Tomography \\
\hline DC & Direct Current \\
\hline DFT & Defibrillation Threshold \\
\hline EIDORS & Electrical Impedance and Diffuse Optics Reconstruction Software \\
\hline ERP & Event Review Pro \\
\hline FDM & Finite Difference Method \\
\hline FEM & Finite Element Method \\
\hline HI & Heterogeneity Index \\
\hline HSD & Honestly Significant Difference \\
\hline MCM & Monte Carlo Method \\
\hline MM & Moments Method \\
\hline RMSE & Root Mean Square Error \\
\hline SA & Sino-Atrial \\
\hline SSE & Sum of Square Error \\
\hline TTI & Transthoracic Impedance \\
\hline ULV & Upper Level of Vulnerability \\
\hline UOHI & University of Ottawa Heart Institute \\
\hline VF & Ventricular Fibrillation \\
\hline VT & Ventricular Tachycardia \\
\hline & \\
\hline
\end{tabular}




\section{Introduction}

Synchronized electrical cardioversion is a treatment that applies electric pulses to restore sinus rhythm in patients presenting atrial fibrillation (AF), atrial flutter (AFL) or ventricular tachycardia (VT). Since the 1950s, this electrical therapy has been used for treating cardiac arrhythmias, and studies thereafter have shown that the success of this treatment is largely dependent on two factors:

1) Length of time the patient has been experiencing cardiac arrhythmia [1][2].

2) Amplitude/ Magnitude of electrical current reaching the heart [3][4][5].

The monophasic defibrillators that were traditionally used during cardioversion were found to be limited in their efficacy. This first direct current (DC) defibrillator required higher energy and current to successfully defibrillate the patients, and mostly resulted in cardiac damage [6]. Also, their success rate was highly affected by the transthoracic impedance (TTI), with reduced success for patients exhibiting high TTI [2].

A successful defibrillation requires depolarization of $95 \%$ of the heart cells. Therefore TTI has become an important predictor for successful defibrillation, since the current reaching the heart to achieve this depolarization is inversely related to impedance [7]. While a high amplitude current shock ensures depolarization of the myocardium, it may cause damage to the cardiac tissue [5]. Thus, a successful cardioversion entails lower $T T I$ and delivery of an optimal current based on the patient's TTI. The monophasic defibrillators assumed the patient impedance as $50 \Omega$ and did not attempt to adjust the current waveform in regard to the variation in patient's TTI. As a result, higher energy was required for a successful defibrillation, which was also reported to result in myocardial injuries. These limitations led to the introduction of impedance-compensating biphasic defibrillators in late 1980s [8]. 
Since then it has been used for cardioversion and defibrillation, wherein the device varies the duration of the waveform depending on the impedance of the patient [9].

\subsection{Motivation}

With the advent of biphasic defibrillators, studies have compared monophasic and biphasic waveforms and it has unequivocally been shown that biphasic is superior to monophasic. The biphasic defibrillators required lesser energy to defibrillate with less cardiac damage and dysfunction [6-9]. Studies have advocated that biphasic defibrillators are efficient enough to no longer consider TTI to ensure the success of cardioversion [11]. However, several publications contradict these results and shows that although biphasic devices defibrillates at lower energy, it is just as inefficacious for higher TTI as monophasic devices [12]. The outcome of biphasic defibrillators being dependent on the impedance, thereby improving the success rate of the treatment, is still unclear.

Due to this ambiguity, the clinical cardioversion protocol does not take into consideration the TTI of the patient. As a result, it has been observed that a greater number of shocks is required for patients with high $\pi I$, thereby reducing the chances of successful defibrillation[13]. If it can be demonstrated that impedance affects the cardioversion success rate, its measurement could be incorporated into the defibrillation technology to vary the delivered current based on the patient's $\pi I$. This would not only result in delivering the optimal current required for successful defibrillation for any impedance value but also permit a reduction in the number of shocks delivered, both of which ultimately increase the positive outcome of cardioversion. 


\subsection{Thesis Objective}

While there is a strong empirical evidence to support biphasic waveforms for defibrillation and cardioversion, there is a limited understanding with respect to patient outcomes for wide spectrum of TTI values. The objective of this thesis aims to examine the impact of TTI on the success of cardioversion. To illustrate the effect, we have divided our work into two parts. In the first part, retrospective data gathered during the clinical treatment/use of Philips HeartStart XL and Heartstart MRX defibrillators at the University of Ottawa Heart Institute (UOHI) are used to statistically investigate TI's effect on cardioversion outcomes. The second part deals with 3-D modelling of the human thorax using Finite Element Method (FEM) to show the current density distribution for subjects with high, average and low TTI. The model is built using computed axial tomography (CAT) scans of the human thorax to try to ensure an anatomically realistic model. This 3-D FEM enables quantitative assessment of the current distribution pattern during cardioversion, to help explain observations made in the retrospective analysis. In this thesis, we also seek to examine the effect different positions of the pads have on the success of cardioversion using FEM. There is disagreement among the researchers and physicians over the effect of pad position and most suitable position for cardioversion. Therefore, if we could find the position that yields higher success, it could serve as one of the method to increase the efficacy of cardioversion.

\subsection{Thesis Contributions}

The major contributions of this thesis are:

1. Completed a statistical analysis to examine the effect of $\pi I$ on the efficacy of cardioversion.

The effect of transthoracic impedance on the success rate of cardioversion was examined using Fischer's exact test and Chi-square test at significance level of $\alpha=0.05$. With 1253 number of shocks obtained for AF, AFL, and VT, our results 
showed statistically significant difference between the success rate of low and high impedance patients at $\mathrm{p}$-value of 0.04 and 0.006 for AF and $\mathrm{VT}$ respectively. The success rate was higher for low impedance patients in both these arrhythmias with $22 \%$ more success at low impedance for AF and $45 \%$ more for VT. However, the success for AFL was more than $90 \%$ for both impedance levels. Statistically, no significant relationship ( $p$-value of 0.11 ) was observed between the success rate and impedance for this arrhythmia. This demonstrates that even with the modern impedance compensating biphasic defibrillators, $\pi I$ value affects the performance of cardioversion.

2. Determined the range of current amplitude that should be delivered for a successful cardioversion.

We have examined the relationship between current and shock success in successful cardioversions. The results show the optimal current range for a successful cardioversion lies between 28 to 48 A for AF and 24 to 32 A for VT. Current less than $16 \mathrm{~A}$, and greater than $48 \mathrm{~A}$ for AF and $32 \mathrm{~A}$ for VT results in decreasing the efficacy of the treatment. We also found that for AFL, even low amplitude of current $(<16 \mathrm{~A})$ would result in a successful cardioversion.

3. Examined the effect of TTI on current density distribution in the thorax and defibrillation success using FEM on different patient types.

Defibrillation efficacy on patients of different sizes has been examined to analyse the effect of TTI on success rate. CAT scans of thin, normal and large sized healthy people were taken and FEM model were developed based on the CAT scans. Successful defibrillation parameters were calculated and it was found that larger people exhibiting high TII had lower current density in the heart, thus requiring more current and energy for defibrillation than a normal and thin sized person. The results therefore suggest that high current should be delivered to larger patients. Since in a clinical scenario, biphasic defibrillator can deliver current amplitude up to a 
maximum threshold, the available current is insufficient to defibrillate patients with high TTI. This may result in lower defibrillation success rates for such patients.

\section{Evaluated the effect of pad positions used during cardioversion.}

In our thesis, we also examined the effect of pad position on cardioversion. With our results demonstrating that $\mathrm{TI}$ influences the efficacy of defibrillation, we investigated methods to reduce the impedance to achieve higher success rate during cardioversion. Among many factors that influence the $\pi \mathrm{Tl}$, one of the factors is pad position. Three positions, Antero-Lateral (AL), Antero-Posterior1 (AP1), and AnteroPosterior2 (AP2) that are predominantly used during cardioversion were considered for this analysis. FEM models using a CAT scan for a normal healthy male was constructed and each of these positions were simulated in the model. When defibrillation parameters were calculated it was seen that $A L$ position will result in lower performance during cardioversion as compared to both AP positions. Comparatively, lower TI, and lower current and energy requirement for successful defibrillation was observed for AP2 with greater uniformity in the current distribution within the heart. These results demonstrate that pad position affects the efficacy of cardioversion and AP2 position could yield higher success rate. Our analysis also suggests that the defibrillation parameters like energy, current and resistance can be sensitive to pad placement errors done by human operators during a clinical cardioversion procedure.

Since our results showed that different patient types/sizes and pad positions result in different electrical activity within the body, we analysed the effect of pad position on different patient types. We compared the defibrillation parameters calculated for each of the sizes during the three different positions. Our results indicated that cardioverting patients of any size in AP2 position require less current and energy 
than AL or AP1. Even for large patients, successful cardioversion could be achieved with lower current in AP2 position.

Additional minor contributions of this thesis are:

1. Modified the existing FEM algorithm to develop a human chest mesh incorporating heart, skeletal structures, and sub-cutaneous fat tissue. The model was also modified to incorporate different positions of the pads used during cardioversion.

2. Modified and implemented Electrical Impedance and Diffuse Optics Reconstruction Software (EIDORS) algorithm to include different conductivities of the tissues and calculate various defibrillation parameters such as current density in the heart, minimum current and energy required for a successful defibrillation, uniformity in the current distribution and percentage of cardiac damage.

The statistical and FEM results shown in this work clearly indicates the effect of TTI on the efficacy of cardioversion. We also suggest a way to improve the success rate of this treatment by placing the pads in AP2 position.

Part of this thesis has been disseminated in the following publication:

Chaugai V, Adler A, Chan ADC, Zakutney T, "Estimation of effective pad positions during cardioversion using 3-dimensional finite element model", $35^{\text {th }}$ Conference of the Canadian Medical \& Biological Engineering Society, Halifax, Canada, 2012. 


\subsection{Organization of Thesis}

Chapter 2 of this thesis reviews the background information on cardioversion, and theory behind fibrillation and defibrillation. Chapter 3 covers the cardioversion procedure, data acquisition, data processing and experimental measurement of voltage to calculate the current. Chapter 4 describes retrospective statistical analysis of our clinical data and the results. Chapter 5 deals with the mathematical fundamentals of FEM. Chapter 6 describes the 3-D model of the human thorax, analysis of current distribution for different types of patients and the effect of pad position on the behavior of the current in the body. Chapter 7 summaries the thesis and discusses potential future works. 


\section{Literature Review}

\subsection{Introduction}

Chapter-2 provides a background regarding the physiology of the heart and cardiac arrhythmias, and a brief overview of the relevant research in the field of cardioversion. This includes physiology of fibrillation and defibrillation, evolution of defibrillation technology and several challenges that still prevail for a successful defibrillation of the heart.

\subsection{Conduction System of the heart}

The heart is divided into four chambers, the upper atria and lower ventricles. Located in the right atrium is the Sino-Atrial (SA) node, which is often referred to as the natural pacemaker of the heart. The SA node spontaneously fires electrical impulses $(60-100$ impulses per minute) that initiate heart contractions.

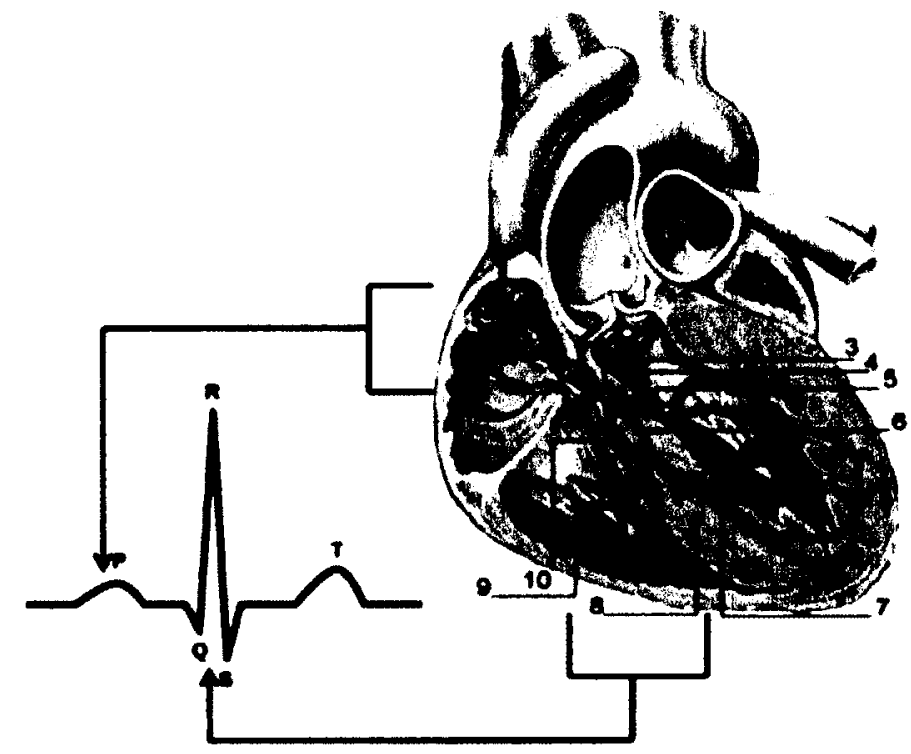

1. Sinoatrial node

2. Arrioventricular node

3. Bundle of His

4. Left Bundle branch

5. Left anterior fascicle

6. Left posterior fascicte

7. Left ventricle

8. Ventricular septum

9. Right ventricle

10. Right bundke branch

Figure 2.1 Electrical Conduction System of the Heart. Credit: Patrick J. Lynch, medical illustrator; C. Carl Jaffe, MD, cardiologist. http://creativecommons.org /licenses/by/2.5 
The impulse initiated by the SA node travels in the atria causing the atrial muscles to contract. This causes the blood from the atria to be ejected into the ventricles. Since the atria and the ventricles are electrically isolated, the impulse propagates to the ventricles via the Atrio-Ventriuclar (AV) node. Electrical conduction is delayed through the AV node, allowing the atria to empty the blood into the ventricles before the ventricles contract. The impulse then travels down from AV node, through the Bundle of His, bundle branches, and then to the Purkinje fibers, which innervate the ventricular cells. The ventricles then contract and pump the blood either to the lungs for oxygenation (right ventricle) or to the rest of the body (left ventricle). Comprehensive information regarding the electrophysiology of the heart can be found in [14].

\subsection{Cardiac arrhythmia}

Cardiac arrhythmia refers to any abnormal electrical activity in the heart which causes irregular heartbeats. The fundamental cause of most of the cardiac arrhythmias is coronary artery disease [14]. Also, diseased heart valve(s), cardiomyopathy, ventricular hypertrophy and arterial embolism may contribute in generation and transmission of abnormal impulses in the heart leading to arrhythmia [14]. Cardiac arrhythmias may prevent the heart from efficiently pumping the blood to the rest of the body, which at first causes an increase in the cardiac work and ultimately damages the brain and other organs by not providing sufficient oxygenated blood. Comprehensive information regarding cardiac arrhythmias can be found in [14].

\subsubsection{Types of Cardiac Arrhythmia}

Generally, the types of cardiac arrhythmias have been divided into four categories,

1. Premature beats: Premature beats are naturally occurring arrhythmia characterized by a feeling of a skipped heartbeat .Premature beats are harmless in general. 
2. Bradyarrhythmias: Bradyarrhythmias occur when the heart rate is slower than 60 beats per minute. Normal heart rhythm is restored using either a pacemaker or a defibrillator providing pacing assistance.

3. Supraventricular arrhythmias: This includes atrial fibrillation, atrial flutter and paroxysmal supraventricular tachycardia. These arrhythmias can be treated by synchronized cardioversion.

4. Ventricular arrhythmias: Ventricular tachycardia and ventricular fibrillation fall under this category. Defibrillation is generally done for patients suffering from this type of arrhythmia.

Among these four conditions, supra ventricular arrhythmias and ventricular arrhythmias pose serious threats where the latter leads to imminent death if not treated on time ( $\sim 1$ to 3 minutes) $[14]$.

\subsubsection{Physiology of Cardiac Arrhythmia}

The occurrence of arrhythmias in the heart has been explained with the following two theories: 1) Ectopic Foci or 2) Circus Movement or Re-entrant circuit.

Regions in the heart, other than the SA node, may also generate electrical impulses; those areas are termed ectopic foci. The impulses generated from these foci are usually suppressed due to higher impulse rate of SA node. However, the occurrence of ectopic impulses during the relative refractory period of the cells, also referred as vulnerable period, as shown in Figure 2.2, can spread disorganized depolarization waves causing irregular heart rhythm [14]. In a normal healthy person, an ectopic pulse occurring during the Twave is well tolerated by the heart and SA regains its control. However in diseased heart, these areas, if stimulated, takes over the control of SA node and send rapid impulses in all directions resulting in flutter or fibrillation [14][15]. 
During the end of a normal cardiac cycle, an impulse generated by the SA node eventually dies out due to the refractory state of the cells and a new excitation impulse is again generated from the SA node. There exist conditions for which the propagating pulse does not die out after the excitation of the heart and continues to propagate in a circle to reexcite the heart cells immediately after the repolarization [14]. This cyclic propagation leading to the re-entry of the impulse over and over again is described as circus movement. This leads to a sustained abnormal cardiac rhythm that ignores the pacesetting function of the SA node. There are three conditions that facilitate this re-entry of the impulse [14]:

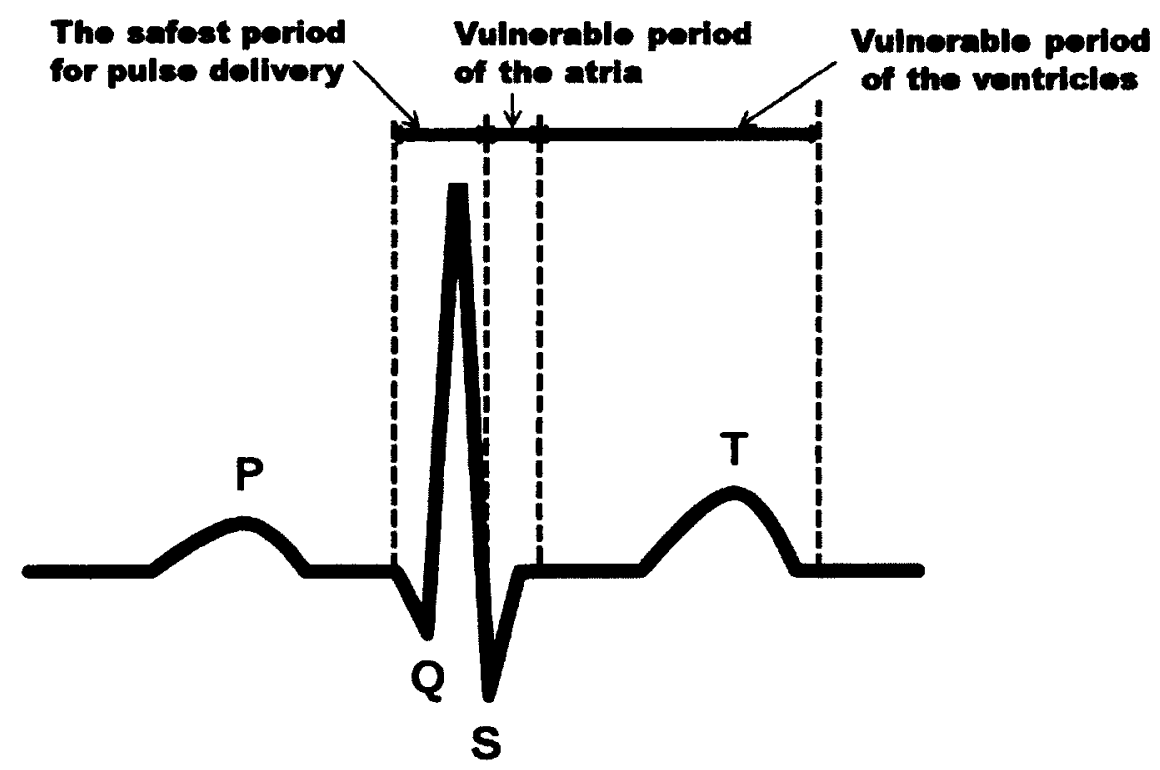

Figure 2.2 Vulnerable period during sinus rhythm

a) Longer length of the conduction pathway: This is basically seen in dilated hearts. Figure 2.3 shows propagation of the impulse in a longer conduction pathway.

b) Slow conduction velocity of the impulse: This result from ischemia, elevated blood potassium or any blockage in the Purkinje fibres.

c) Shortened refractory period: Consumption of drugs such as epinephrine could cause shortening of the refractory period of the cells. 
Supra ventricular arrhythmia and ventricular arrhythmia are believed to occur as a result of either ectopic foci or re-entry or a combination of both. Although these theories attempt to explain the phenomena behind the arrhythmias, a definitive mechanism has yet to be defined.
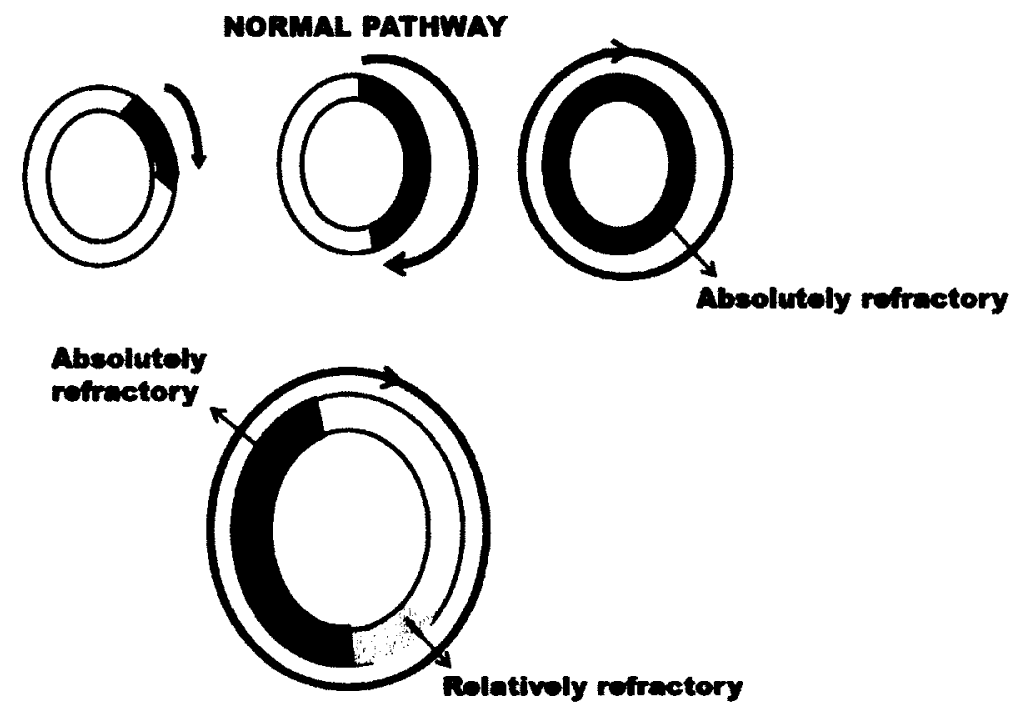

LONG PATHWAY

Figure 2.3 Re-entrant circuit as a result of long pathway. Based on [14].

\subsection{Theory of defibrillation}

The main therapy for cardiac arrhythmias is defibrillation and/or cardioversion. Defibrillation is carried out in an emergency condition for patients under ventricular fibrillation (VF). Cardioversion is done for patients exhibiting atrial fibrillation (Afib), atrial flutter (AF) and ventricular tachycardia (VT). Cardioversion is done on a scheduled basis and in a synchronized way, i.e. the shock is given only during the R-wave in the ECG pulse. This is done to avoid any occurrence of cardiac arrest or stroke. Although the procedure and conditions for defibrillation and cardioversion are different, both these treatments follow the same principle. At a general level, defibrillation and cardioversion works by depolarizing the heart cells to defibrillate the patient's heart and restore a normal sinus rhythm. 
Even though the basic idea behind defibrillation is known, the underlying mechanism still remains unclear. There exist many theories that attempt to describe the effect of the shock on the myocardial tissue and their relationship to the shock success, each of them interrelated and complimentary to one another. Among many of the hypotheses, four of those remain popular in explaining the mechanism for defibrillation.

1. Critical Mass Theory: This theory proposed by Zipes [16] explains that a shock need not stop all the irregular impulses propagating in the heart. For a successful defibrillation, it is only necessary to halt the activation wave front occurring in a certain critical mass of myocardial cells such that the remaining mass of fibrillating tissue would be insufficient to sustain the arrhythmia. Studies have reported that at least $95 \%$ of the cells have to be defibrillated for a successful outcome [16][17].

2. Upper Level of Vulnerability (ULV): Chen et al. [18] observed different patterns of post-shock activation wave fronts in the successful and failed defibrillation which led to the formation of this theory. During defibrillation, the regions of low shock strength (areas further away from the stimulating electrode) may get stimulated during their vulnerable period and give rise to the new activation wave front, thus reinitiating fibrillation. Hence, in order to successfully defibrillate the heart, it is necessary for the shock strength to be greater than the largest strength of shock that could initiate the fibrillation, defined as the upper limit of vulnerability [18]. It has been found that upper limit of vulnerability for the monophasic shocks is $6 \mathrm{~V} / \mathrm{cm}$ and for biphasic is $5 \mathrm{~V} / \mathrm{cm}[19]$.

3. Refractory period extension theory: Defibrillation is said to be successful only when the fibrillating wave fronts can be stopped. Fibrillation wave fronts, in turn can be stopped by blocking their propagation, and the only way of achieving this is to render the cells in their refractory period such that the wave fronts cannot re-enter again. This is the concept behind refractory extension theory which states that a critical shock of optimum duration and strength when delivered should extend the refractory 
period of the excitable cardiac cells so that these cells will not be depolarized by the incoming wave fronts [20][21]. Other remaining cells which are already in their absolute refractory period will remain refractory to the fibrillating impulses. This will not only cause the halting of the activation wave fronts but also puts all the celis into their refractory state making them synchronize at one phase. This will then cause SA node to regain its control and produce normal impulses. Taking into account the ULV hypothesis, for a shock to be successful, it is required that voltage gradient of $5-6 \mathrm{~V} / \mathrm{cm}$ be produced in low intensity regions with an optimal duration so as to allow sufficient time for the cells to remain refractory. This postulate incorporates both the critical mass hypothesis and ULV hypothesis making it the one of the most acceptable theory for defibrillation.

4. Virtual Electrode Hypothesis: This hypothesis predicts that application of a positive stimulus will generate a region of hyperpolarization (virtual anode) and two regions of depolarized tissue (virtual cathodes) [22]. The depolarization tends to propagate outward the virtual cathode region. Simultaneously, the regions under virtual anode will hyperpolarize the tissue and there is no propagation of action potential from those areas [23]. Now, when the propagating depolarizing pulse reaches the virtual anode region, the charge is not sufficient to induce depolarization, as the cells are in their hyperpolarizing state making the fibrillating wave fronts die out. The stimulating pulse, however, has to be applied for a sufficient duration of time, as the decay of hyperpolarization is very rapid which may cause the cell to be depolarized by the impulse propagating from the virtual cathode region [22].

These four hypotheses are not mutually exclusive. They all agree to the underlying idea that current density during stimulation should be sufficient enough to make all the atrial cells (in case of atrial arrhythmia) or ventricular cells (for ventricular arrhythmia) under refractory period so that the fibrillation wave fronts cannot propagate further and fibrillation stops. 


\subsection{Defibrillation in clinical practice}

Since 1900s, experiments have been carried out for defibrillating the heart using electrical shock. However, it was used clinically on a human only in 1948, when Dr. Claude Beck of Case Western University performed internal defibrillation using alternating current $(\mathrm{AC})$. In mid 1950's, Dr. Paul Zoll first performed external defibrillation on a human by applying AC across externally placed paddles. The external direct current (DC) defibrillation came into practice only in late 1950's when Lown [24] investigated the application and advantage of $D C$ shock over AC. Their results showed DC to deliver high peak current of shorter duration, resulting in less post-shock myocardial damage and higher success rate than $A C$.

These observations led to the use of DC defibrillators, which electrically was an RLC circuit. A DC defibrillator uses capacitors, charged up to a high voltage level ( $1000 \mathrm{~V}$ to $2000 \mathrm{~V}$ ). It is then discharged on the patient's chest via an inductor to deliver a damped waveform of optimum duration ( $5 \mathrm{~ms}$ ) with an energy dose around 100 to $360 \mathrm{~J}$. DC defibrillators since then have become a standard, widely accepted device for defibrillation and cardioversion.

\subsection{Defibrillation technology}

A typical DC defibrillator comprises of a power supply, capacitor and inductor (Figure 2.4). The AC power supply voltage ( $120 \mathrm{~V}$ in North America) is converted to a higher voltage using a step-up transformer. This variable transformer allows the voltage across the capacitor to vary according to the energy selected by the operator. A rectifier then converts this $A C$ voltage to DC voltage. Electrical energy is stored in the capacitor, which is then discharged to the patient. The function of the capacitor in a defibrillator device can be explained by Figure 2.4 
When switch (S) is in position 1, the DC current coming from the power supply unit builds up charge across the capacitor until the voltage of the capacitor equals the source..Activating the switch, by moving it into position 2, enables the current to flow through the inductor into the paddles that are applied on a patient's chest, thus delivering the stored energy.

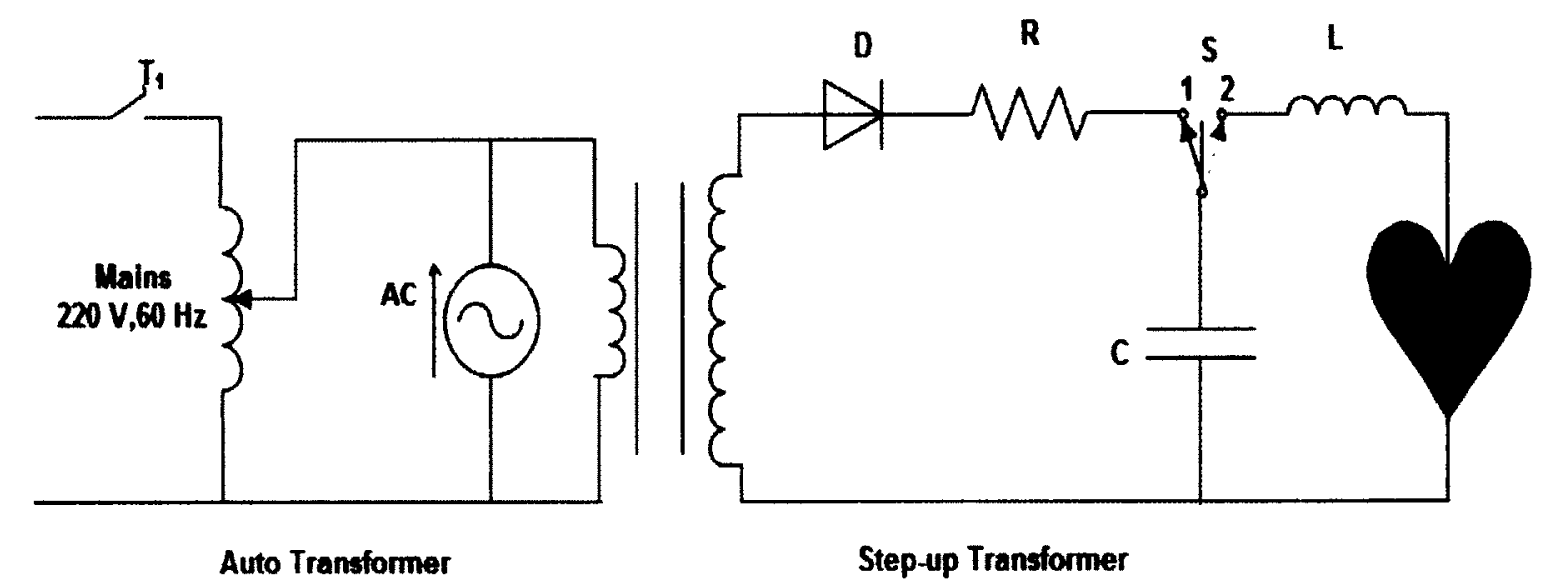

Figure 2.4 Circuit diagram of a DC Defibrillator. Based on http://coep.vlab.co.in

Without the inductor, the decline in the voltage across the capacitor plates during the discharge occurs in an exponential manner, with more energy delivered to the heart in the beginning of the discharge. Therefore, in order to maintain a more steady flow of energy, an inductor is used in the circuit. It minimises the rapid exponential decay of the current and also allows it to flow for a finite duration.

\subsubsection{Defibrillator waveforms}

The shape of the waveform and how the energy is delivered is more important for a successful defibrillation than how much energy is delivered [25]. Defibrillator machines have incorporated two types of waveform (Figure 2.5): 1) monophasic waveforms, where the current travels only in one direction, and 2) biphasic waveforms, where the current traverses in both the positive and negative direction. 


\subsubsection{Clinical Studies Comparing Monophasic and Biphasic Waveforms}

The damped sinusoidal waveform (Figure 2.5) that was delivered during the advent of DC defibrillator in late 1950's was a monophasic waveform or a Lown waveform which was the mainstay for the defibrillator devices for almost three decades [24].
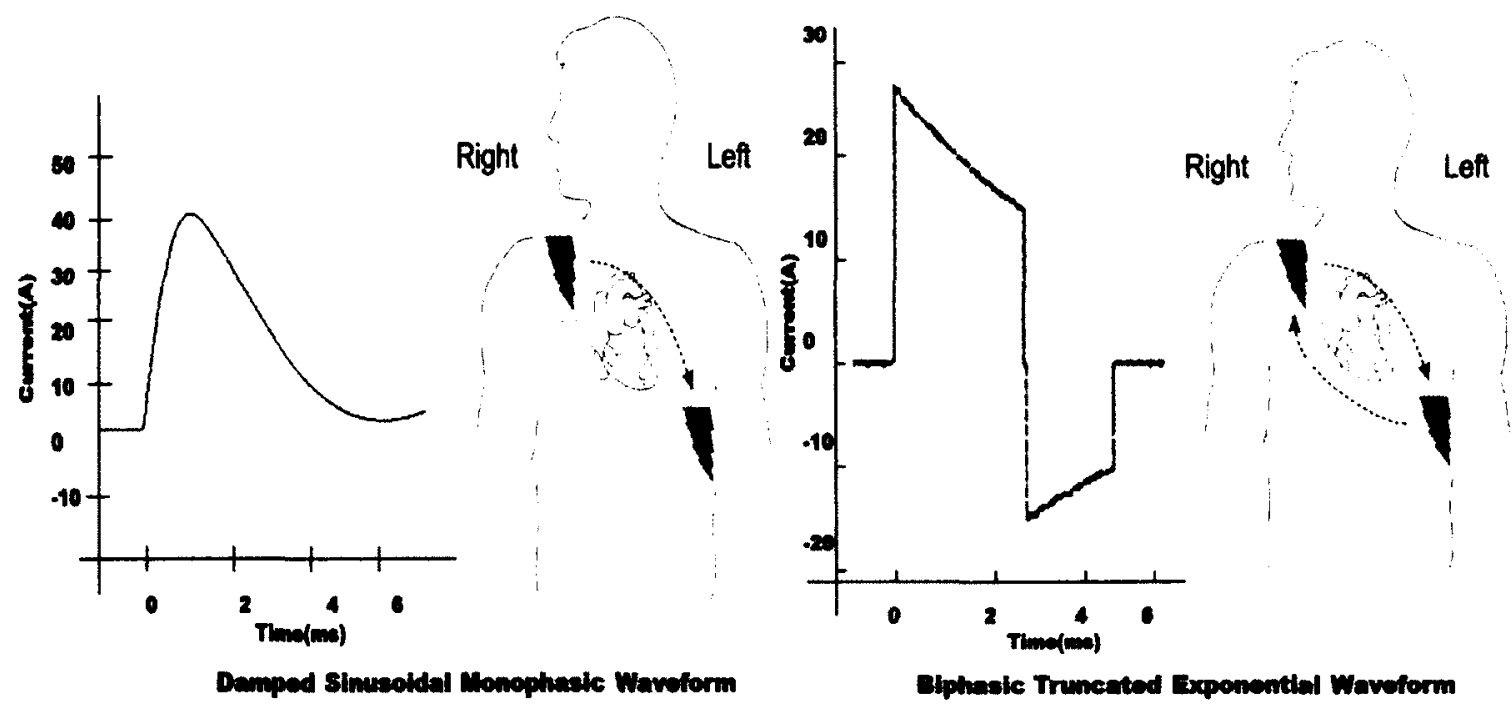

Figure 2.5: Defibrillator Waveforms

In late 1980's, Jones and Jones [8] were the first to report that biphasic waveform tend to reduce the cardiac damage seen during defibrillation. Since then, various studies have been carried out to evaluate the efficacy of monophasic and biphasic waveforms with results unequivocally showing the superiority of biphasic waveform over monophasic [26-36]. Many similar studies have been done to compare the efficacy of monophasic and biphasic, all demonstrating superior efficacy of biphasic shocks at a lower energy level. Table 2.1 provides summary of such works. 
Table 2.1 Comparison of Monophasic waveform (MW) over Biphasic waveform (BW) for cardioversion [37]

\begin{tabular}{|c|c|c|c|c|c|}
\hline Work & $\begin{array}{l}\text { MW energy } \\
\text { selection }\end{array}$ & Success & BW Device & $\begin{array}{c}\text { BW } \\
\text { energy } \\
\text { selection }\end{array}$ & Success \\
\hline 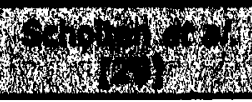 & Why & Wx+m & W & 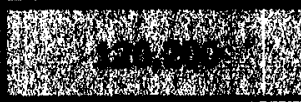 & 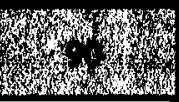 \\
\hline Mittal et al. [38] & $100,200,300,360$ & 79 & $\begin{array}{c}\text { Zoll } \\
\text { PD-2100 }\end{array}$ & $70,120,150,170$ & 94 \\
\hline 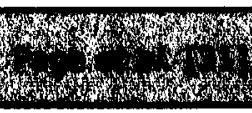 & 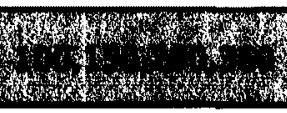 & 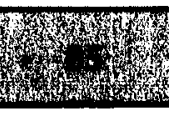 & 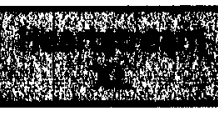 & W7 & 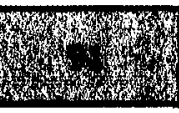 \\
\hline $\begin{array}{c}\text { Marinsek et al. } \\
\text { [35] }\end{array}$ & $100,200,300,360$ & 90 & $\begin{array}{c}\text { Heartstream } \\
\text { XL }\end{array}$ & $70,100,150,200$ & 88 \\
\hline
\end{tabular}

The advantages of biphasic defibrillators reported from all these studies were:

a. Lower defibrillation threshold (i.e., lower amount of current and energy required to defibrillate the heart than monophasic waveforms).

b. Low post shock cardiac damage.

c. Lower energy of biphasic as effective as higher energy of monophasic waveforms.

d. Impedance compensation: Impedance compensation was also studied for monophasic waveforms where impedance compensating monophasic defibrillators yielded more success than the standard defibrillators [3].

These advantages over monophasic waveforms were observed on conditions when the duration of first phase of biphasic was greater than the second phase and for an energy level between $100 \mathrm{~J}$ to $200 \mathrm{~J}$ [38][39]. It was found that both these waveform yielded the same shock efficacy at lower energy level (less than $100 \mathrm{~J}$ ), at a higher energy level (200 J for biphasic and $360 \mathrm{~J}$ for monophasic) and for a lower impedance value [28][31][33][38]. It was also observed that if the duration of first phase of biphasic was less than the second phase, the shock was unable to defibrillate the heart [33]. Research done by Hillsley et al. [40] and Walcott et al. [34] showed that the duration of the first phase should be between 
3.5 to $6 \mathrm{~ms}$ for a successful defibrillation and the waveform should be asymmetrical for higher efficacy.

\subsubsection{Theoretical Comparison of monophasic and biphasic waveforms}

The physiological mechanism behind successful defibrillation by asymmetric biphasic waveform has been attempted to explain by different hypothesis, such as:

1. Charge Burping theory (First phase as conditioning pulse): This is the most accepted hypothesis which suggest that the first phase of the waveform acts as a preconditioning pulse which hyperpolarizes (or repolarizes) the cells [41].

Kavangh et al. [27] demonstrated that the anodal pulse hyperpolarizes the cell towards the membrane potential of $-75 \mathrm{mV}$ to $-85 \mathrm{mV}$, which allows time and voltage dependent sodium channels to recover from inactivation, thus increasing the availability of the sodium channels. The second phase then requires sufficient charge to depolarize the membrane back to its resting potential $(-60 \mathrm{mV})$. Hence, the first positive pulse acts as a pre-conditioning pulse that activates sodium channels and the second pulse acts as an excitation pulse which removes the excessive charge provided to the cells by the first pulse. This theory also explains the importance of the duration of the waveform. The sodium channels that are activated during hyperpolarization are time and voltage dependent. Therefore, if the duration of the first phase of the shock is small, fewer numbers of sodium channels will be activated, which would require larger amount of current (increase in defibrillation threshold) to polarize the cells to $-60 \mathrm{mV}$ [20]. Also, larger second phase duration prolongs membrane breakdown which potentiates myocardial dysfunction.

2. Post shock dysfunction reduction: It has been observed that the post shock arrhythmia is a result of formation of membrane microlesions of diameter $50 \AA$ at regions of high current density [42]. Electrically, a membrane resembles a capacitor and a resistor. So, when a large current intensity is delivered to the cell, electrical 
charge starts to build up across the membrane. When this increases the extracellular membrane potential above a critical value, reversible dielectric breakdown of the membrane occurs which leads to the formation of the pores, thereby making the membrane extremely conductive to allow exchange of ions through these pores. This rapid, indiscriminate exchange of ions, results in depolarisation of the cell leading to the formation of a conduction block which may ultimately lead to a temporary arrest or re-initiate fibrillation or cause irregular contractions if it persists for a longer period of time [20]. Referring to the explanation above, during monophasic shock, hyperpolarization pulse induces membrane microlesion formation. This microlesion is transient and it ultimately reseals itself. The self discharge process which depends upon membrane time constant is slow and increases the chance of cardiac dysfunction. With biphasic waveforms, the reversed polarity of the shock quickly discharges the membrane capacitance. This gives lesser time for ionic exchange through the pores, thereby decreasing post shock dysfunction. Thus it is required that, for a successful defibrillation, the phase duration should be large enough to render the cells refractory for the incoming wave fronts but not too large to induce myocardial damage. The optimal duration for a waveform varies according to the strength-duration relationship for each device, with most studies reporting between 6 to $10 \mathrm{~ms}$ for biphasic waveform [36][39][41].

\subsection{Effect of transthoracic impedance}

For defibrillation to occur, it is necessary that a significant mass $(95 \%)$ of the myocardium cells are depolarized. The mass of cells undergoing depolarization is significantly influenced by the amount of current reaching the myocardium. Not only the magnitude of current, but the spatial distribution of the current density in the heart also affects the defibrillation efficacy since a non-uniform current density distribution has a higher chance of re-inducing arrhythmia, thus leading to refibrillation [43]. This current density in the heart is mostly 
dependent on transthoracic impedance (TTI) [3][4][7][44]. TTI which has been recognized as the most important element for defibrillation is the thoracic impedance or the chest impedance of a person. The influence of TII on current can be shown by the Ohm's law equation

$$
I=\frac{V}{R_{T T I}}
$$

Where I is the current, $V$ is the voltage and $R_{T T I}$ is the chest resistance (clinically referred as transthoracic impedance).

It can be understood that for a given voltage, high $\pi T$ allows a small amount of current to flow, which may not be sufficient to defibrillate the heart. Giving higher dose of current will resolve this problem; however, such intensity may result in damaging the myocardial tissue or induce post-shock dysfunction. Therefore, for sufficient current to reach the heart for a successful defibrillation, lower TTI is desirable. TI is largely affected by anthropometric factors like chest size, phase of ventilation, amount of fat, hair coverage and factors related to technique and defibrillator like paddle force, pad size, pad position, skin coupling gel, number of shocks and the interval between each shock, each described in detail below.

1. Paddle Contact Force: Electrode-skin impedance largely contributes to the total impedance of the body. A firm paddle force ensures a good electrode-skin contact, and reduces the skin impedance and also reduces the thoracic volume, both of these conditions resulting in overall decrease of the TTI. Deakin et al. [45] conducted a study on paddle force that should be applied during defibrillation and they found that applying at least $78.45 \mathrm{~N}$ force on adult patients, which is attainable by $80 \%$ defibrillator operators, reduced the $\pi I$ by a mean of $12.63 \Omega$. In children, (1 to 17 years) force should be approximately $49 \mathrm{~N}$ and $29.4 \mathrm{~N}$ for babies [46].

2. Coupling gel: Application of a coupling gel, just like the high paddle force, decreases the skin contact impedance. Although the application of these gels ensures good electrical contact, use of these gels will create a low impedance pathway along the 
chest wall. This will cause very little current to flow to the heart with most of it flowing along the chest wall, forming an electric arc between the electrodes [46]. To reduce the smearing of the gels, it is advisable to use gel-pads for hand held paddles during defibrillation which provides good electrical contact and are easy to apply and minimizes this risk.

3. Body Hair: Chest hair increases the contact impedance due to the poor electrode contact and air trapping. Defibrillation on an unshaved chest has higher chances of burns than on shaved ones. Shaving off the hair minimises the TTI.

4. Phase of Ventilation: Ewy et al. [47] performed an experiment on dogs to show the influence of phases of ventilation on defibrillation. They found that there was a TTI difference of approximately $10 \Omega$ between the inspiratory phase and expiratory phase with the former having more impedance due to the presence of air in lungs. They observed $10 \%$ of defibrillation success rate for inspiration and $50 \%$ for expiration concluding that phases of ventilation significantly affect the impedance in turn affecting the defibrillation success rate.

5. Paddle size: Use of larger electrodes will lower the TTI and allow more flow of current. However, using a larger electrode will facilitate larger current path with chances of the current flowing through extra cardiac paths within the thorax, thereby missing the heart. In smaller electrodes, lesser current flow is seen but the electrode area is small, therefore, the current density is higher and chances of tissue injury are possible. Research has shown the optimal paddle size to be around $8.5 \mathrm{~cm}$ to $12 \mathrm{~cm}$ [48].

6. Paddle Placement: Different areas of the chest offer different resistance for the current flow. Electrodes are placed in such a position that heart is located between them. In that way, majority of the current would be flowing through the heart and not the extra cardiac pathway. According to the recommendations from American Heart Association (AHA), the electrodes should be located at the apex-anterior (anterior-lateral, 
Figure 2.7) position such that the anterior electrode is placed at the right sternum and the apical electrode placed on the apex of the heart.

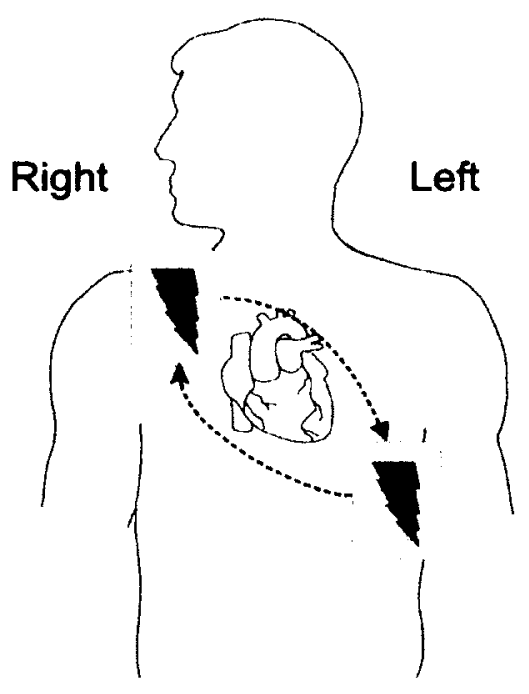

Antero-Lateral (AL)

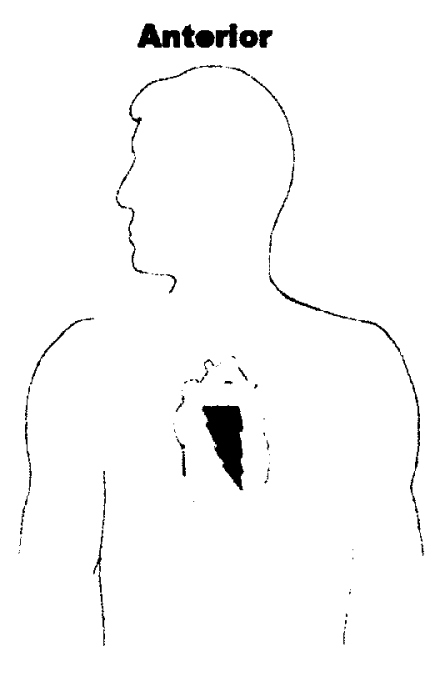

Antero-Posterior1 (AP1)

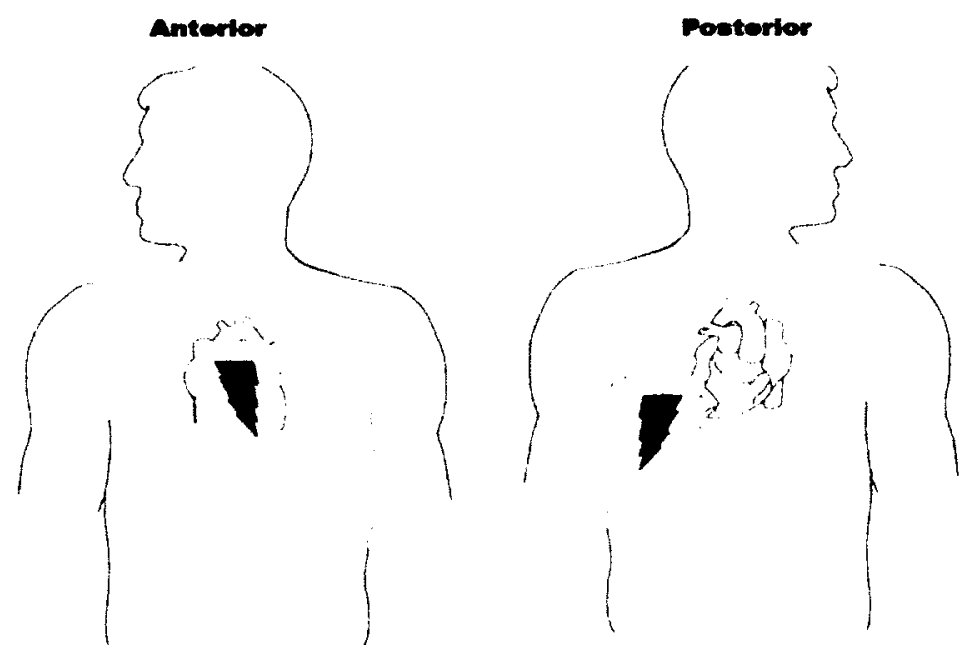

Antero-Posterior2 (AP2)

Figure 2.6 Standard Paddle placement for cardioversion

In a hospital setting, there are three most common pad positions used during cardioversion: 1) Antero-Lateral (AL) position, 2) Antero-Posterior1 (AP1), and 3) Antero-Posterior2 (AP2) (Fig. 2.7). Several studies have been done to estimate the most effective position that results in higher success rate for defibrillation. Some suggest AP position to be more effective than conventional AL position [49-52]. These 
studies have shown lower TTI and higher success at lower energy level for the AP position. However, some of the work carried out on the clinical data reports both the positions to be equally effective, stating that the position of the electrodes does not affect the efficacy of cardioversion [2][7][53]. This uncertainty over the effect of pad placement and the most suitable position has made the protocol for cardioversion clinician specific with some preferring AL and some AP position during the treatment.

These factors lead to variation in the TI during cardioversion which ultimately affects the efficacy of the process from patient to patient, with poor results for high impedance patient [3][4][7]. These observations were seen to be more evident on monophasic devices. This resulted in the implementation of "impedance-compensating" biphasic waveform for defibrillation where the voltage and duration of the waveform changed according to the impedance of the patient [9].

\subsubsection{Impedance compensation}

As much as a sufficient amount of current is important for successful patient outcome, it is also important that the device delivers that amount of current on the first shock. Increasing the number of shocks to more than three exacerbates the fibrillating condition by causing sarcolemma microlesions that damages the myocardial tissue [42]. To take into account the first shock success, biphasic defibrillators were designed such that the waveform compensated for high and low TTI. Different companies have their own standards for compensating impedance for biphasic waveform. We will describe about biphasic technology for the HeartStart XL and HeartStart MRX Philips Medical defibrillators that we have used in this study.

These biphasic defibrillators change the shape of the waveform to maximize its efficacy for each patient as shown in Figure 2.7. Before the shock delivery, the device derives the 
impedance from the voltage and current measurement and adjusts the duration of the waveform to deliver the fixed energy. In this way, lower impedance patients receive higher current for a shorter duration and high impedance patient receive lower peak current for longer duration. Therefore, by adjusting the duration of the waveform the defibrillator compensates for the impedance, thus providing a similar dose of energy to all values of impedances.

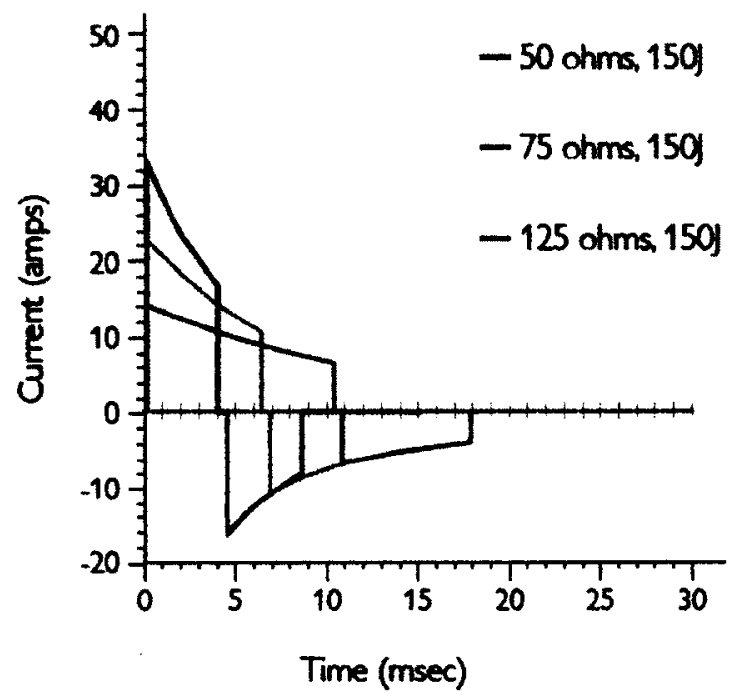

Figure 2.7 Impedance Compensation by HeartStart Philips MRX for energy at $150 \mathrm{~J}$. Reproduced from [9]

\subsubsection{Controversy regarding the impedance compensation of biphasic defibrillators}

The impedance compensated method led researchers and clinicians to believe that differences in TTI no longer affect the success of defibrillation [2][11]. In 2003 Walker et al. [12] compared the efficacy of six different biphasic defibrillator devices on swine and found that despite the impedance compensating schemes, the TTI still affects the effectiveness of defibrillation. Their results were highly criticized and questions regarding their data arose in that the shock dose given to the animals were different to the clinical shock dose received by the patients with higher TTI [54]. Their results were contrary to those obtained by Roger et al. [11], who tested the success rate of $150 \mathrm{~J}$ biphasic 
waveform on impedance from $30 \Omega$ to $165 \Omega$ and showed that $\pi I$ does not affect defibrillation. Instead it was the time interval between the occurrence of fibrillation and first shock delivery that contributed to successful resuscitation of the patients.

With all these advancements in the defibrillator technology, the optimal amount of current and energy dose for defibrillation, the best method of impedance compensation and the effect of $\pi I$ on the biphasic waveform still remain heavily debated.

\subsection{Recent Advancements}

\subsubsection{Finite Element Method (FEM)}

The FEM is a numerical analysis technique that has been extensively used to determine the current density distribution in the thorax during an electrical defibrillation $[51],[52],[55-66]$.

Several numerical techniques have been used to solve the Laplace equation governing the electric potential distribution, amongst which FEM has been found to be the most flexible technique. The modelling of conductivity distribution of the thorax using such 3-D FEM has led researchers to optimize the electrode configuration during defibrillation. The FEM has led to significant findings in the defibrillation technique including the type, the size, and the position of the electrodes. The circular electrodes with pad sizes of $8 \mathrm{~cm}$ to $12 \mathrm{~cm}$ that are incorporated during defibrillation today are a result of FEM outcomes which have been found to improve the defibrillation performance. Detail information on FEM is provided in Chapter 5 and Chapter 6. 


\subsubsection{Triphasic waveforms}

Usage of truncated triphasic exponential waveform is being studied for defibrillation on animal models. These triphasic waveforms basically consist of a combination of biphasic and monophasic wave (i.e., positive, negative and a positive pulse, Figure 2.8 ).

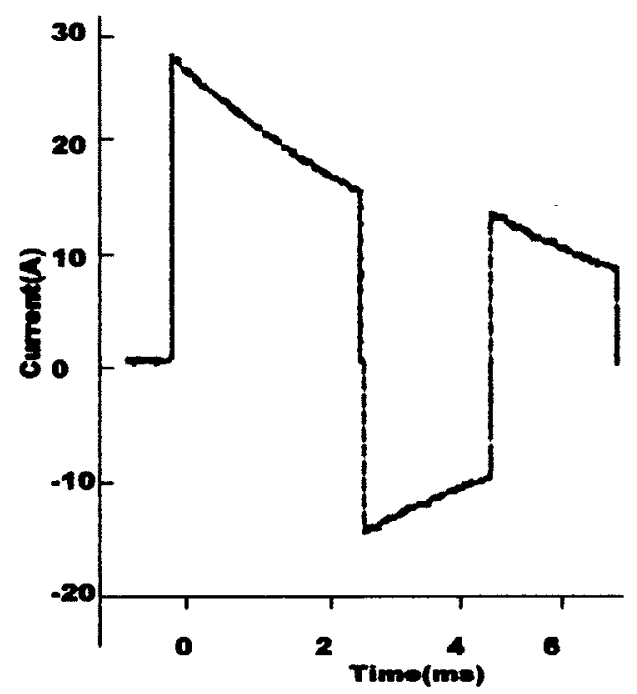

Figure 2.8 Triphasic waveforms used for defibrillation

Zang et al. [67] compared the biphasic and triphasic waveforms on a swine model and found that a triphasic waveform with an equal duration as the biphasic, yields higher success rate at a lower energy level. This results were similar to work done by Jones and Jones [68], where they found superior efficacy of triphasic over biphasic with comparatively less tissue damage. They postulated that the first pulse acts as a "pre-conditioning pulse", second pulse as "exciting" pulse and the third negative pulse as "healing post-pulse" which amended the dysfunction caused by the first two pulses.

The impact of triphasic waveform, at present is still experimental with research limited to only animal models.

\subsubsection{Alternating Current $(\mathrm{AC})$ defibrillation}

In 2008, researchers in Israel built a defibrillator delivering mains-supplied AC shock for the purpose of defibrillating the patients with VF at home [69]. They take this idea of AC shock 
delivery from the initial days of defibrillation when $A C$ was delivered to the heart. The $A C$ device uses high voltage and current switch powered from the AC mains to modulate the current and generate a waveform similar to the DC biphasic waveform. After modulation, the current is conducted to the high voltage transformer, where it amplifies the signal and delivers to the pads. The use of this device was tested on five swine pigs and they found that there was no significant difference in the shock success for $70 \mathrm{~J}$ from the $A C$ defibrillator with that of $65 \mathrm{~J}$ from a standard DC defibrillator. No macroscopic cardiac damage was reported making the $A C$ defibrillator as effective as the $D C$ one.

One of the limitations of this device is that since it functions on AC current, it would be difficult to deliver a biphasic pulse if the AC signal takes other wave shapes due to harmonic distortion.

This low-cost, AC home defibrillator is an interesting concept and it could benefit the patient in cases of emergency conditions, however, more detailed study is required to substantiate its efficacy and safety factor.

\subsection{Summary}

Defibrillation and cardioversion are complicated processes. Understanding their mechanism is indeed required for the development of better protocols to treat patients suffering from arrhythmia. Nonetheless, with the studies that have been conducted so far, it is known that a successful cardioversion largely depends on the duration between the occurrence of fibrillation and delivery of a shock, and the current density. The amount of current in turn depends of the TTI. The monophasic waveforms that were used for 30 years did not account for the change in impedance giving a poor performance. Replacement of the monophasic waveform by the impedance-compensating biphasic waveform was a remarkable leap in the defibrillator technology. However, cases of unsuccessful defibrillation and escalation of 
energy for a high impedance patient are still prevalent for biphasic waveforms. Therefore the effect of impedance on the successful patient outcome during cardioversion remains ambiguous.

This thesis deals with examining the effect of impedance on the outcomes of cardioversion which is carried out in two parts. The first part involves using retrospective data collected at University of Ottawa Heart Institute to statistically determine the significance of impedance on the performance of cardioversion. The methodology and results for this retrospective analysis are described in chapter 3 and chapter 4 respectively.

The second methodology for studying the effect of impedance includes using Finite Element Method to examine the electrical activity in the thorax during cardioversion for subjects with high, average and low impedance. FEM is also used to investigate different positions of the pads that results in higher success with lower defibrillation threshold. The methodology and results for FEM are discussed in chapter 5 and chapter 6 respectively. 


\section{Experimental Methods}

\subsection{Introduction}

This chapter details the cardioversion process at $\mathrm{UOHI}$, how the data are collected after the procedure, and different methods carried out to extract pertinent information from the data. This is done to thoroughly understand how the data are acquired during cardioversion in a hospital setting which will help in interpreting the statistical results.

\subsection{Cardioversion Procedure}

Cardioversion is performed on an outpatient basis in UOHI. At least four (4) weeks before cardioversion, the patient is asked to start anticoagulation medication. This is done to thin the blood since clotting of the blood in the heart during cardioversion can cause a heart attack. The cardioversion treatment requires the presence of a cardiologist, nurse, and an anesthesiologist. Special cardioversion pads (Kendall Medi Trace $1710 \mathrm{H}$ defibrillation electrodes, Figure 6.2 in Chapter 6 ) are placed at either Antero-Posterior or Antero-Lateral position. The selected positions are at the discretion and preference of the cardiologist. These pads are connected to the HeartStart Philips Medical XL defibrillator through which energy for cardioversion is applied. The external defibrillator is also connected to the Philips Intellivue MP30 Physiological monitor that displays blood pressure, breathing, and heart rhythm of the patient. Since a high voltage shock $(>1000 \mathrm{~V})$ is delivered, it is required that the patient is sedated so that he/she does not feel any discomfort. An anesthesiologist administers sedation intravenously and only when the patient is fully unconscious, the cardiologist delivers a shock. The shock during cardioversion is automatically timed by the defibrillator to be delivered only during the R wave. If the patient's ECG returns to normal, the procedure is successful and is stopped. Otherwise, the energy level is increased and the process is repeated. After the treatment, patients tend to wake up quickly and they have no 
recollection of the shocks. In cases when the cardioversion is unsuccessful after three to five repeated shocks, the cardiologist stops the procedure to avoid any post-shock cardiac dysfunction [13]. During such cases, they prescribe medications to keep the heart rhythm in an acceptable range. Sometimes they also suggest medications that will make the tissues more conductive so that sufficient current reaches the heart for next cardioversion session.

\subsection{Data Acquisition}

This retrospective study was carried out with the approval from Research Ethics Board at UOHI and Carleton University. Two (2) types of defibrillators - HeartStart Philips XL and HeartStart Philips MRX are used for defibrillation at UOHI. Both these devices are the same technology, but the MRX device provides many additional diagnostic features and also presents information regarding the amount of current delivered to the patient which is useful for our research work.

Data for this study were acquired from these two devices. When these defibrillators are used during cardioversion, de-identified information such as ECG, amount of energy delivered, number of shocks delivered, impedance of the patient gets automatically recorded in a memory card of the defibrillator. The data in the memory card is then imported into HeartStart Event Review Pro (ERP) v3.3 running remotely on a UOHI server. ERP is a data management software provided by Philips Medical Systems Inc. to electronically manage and review the cases stored in the defibrillators.

Since 2009, 6475 cases have been collected from these defibrillators. The defibrillators record an event as a case file as soon as it is switched on. Therefore, these 6475 data not only include the cardioversion/defibrillation events but also cases that represent situations where a nurse switches on a machine during his/her duty check, equipment maintenance where the machines were used with simulators, usage of the device to view the ECG during 
the transportation of patients between different wards, or any other "non-defibrillation" condition. Since we are interested only in those files that represent cardioversion/defibrillation events, we separate the data using Microsoft SQL server Management Studio Express and further sort it out by inspecting the recorded ECG waveform.

\subsection{Data Processing}

\subsubsection{SQL}

ERP software supports the use of Microsoft SQL Server for managing its database. In this work, Microsoft SQL Server 2008 was used and an ERP database was created in the SQL Server Database. SQL scripts were designed and executed to select only those cases which included shock information i.e., number of shocks greater than 0 , and energy greater than 20 3. Along with that, information required for our analysis including impedance, energy, and number of shocks were also extracted from the database (Appendix-I). Cases which were a result of a nurse's duty check or usage of machine for transporting patients were excluded as they do not contain any shock information (number of shocks equals 0 ). Only those files where energy greater than $20 \mathrm{~J}$ was chosen since we did not want to include cases that represent open heart internal paddle defibrillation. This resulted in reducing the data volume to 1390 , which now include defibrillation and cardioversion events, simulator cases, and also cases recorded during preventive maintenance of the equipment. For our dataset to represent only the cardioversion events, ECG waveforms for each of 1390 cases were then visually inspected.

\subsubsection{ECG Analysis}

Using ERP, the ECG signals of the remaining 1390 files were visually reviewed. Those cases which had no ECG or where the ECG waveform was a straight line were rejected. Also, files 
which showed perfect ECG before the delivery of shock were rejected since they were from a simulator used in troubleshooting purposes during maintenance and inspections. This reduced the number of cases to 881 . To ascertain that these 881 cases represent true cardioversion events, these ECG records were sent to a cardiologist at UOHI for further inspection. The cardiologist not only discerned the true events but also provided us with the information on the type of arrhythmia and the end result of the cardioversion process for our 881 data. Out of 881 cases, 775 represented cardioversion event, 20 represented defibrillation event, and 30 were from a simulator. There were 17 cases where the cardiologist was not able to distinguish the underlying heart rhythm. There were 4 cases of complete cardiac block and were rejected. The arrhythmias and cardiologist annotations for 35 cases were difficult to understand by the author and were sent back for clarification. These cases are being re-reviewed by the cardiologist and are not included in this thesis. For our work, we only considered the 775 cases representing cardioversion events. Among them, 574 cases were identified as atrial fibrillation (AF), 112 cases as atrial flutter (AFL), and 89 cases of ventricular tachycardia (VT).

\subsection{Measurement of voltage and waveform}

Our analysis not only requires knowledge on the type of arrhythmia, success/failure of the cardioversion process, impedance, and energy but also the current delivered during cardioversion. This information about current is useful for determining the optimal amount of biphasic current that is required for a successful cardioversion.

Most of the data $(\sim 99 \%)$ are acquired from the HeartStart XL machine which does not provide any information on the amount of current delivered. Therefore, we calculated the current experimentally for different resistance and energy levels. For this, we connected the HeartStart XL device to a patient defibrillator simulator whose output was connected to a digital oscilloscope (Figure 3.1). The $\mathrm{XL}$ device was charged to $50 \mathrm{~J}, 100 \mathrm{~J}, 150 \mathrm{~J}$ and $200 \mathrm{~J}$ 
and then discharged to the simulator. The waveform and peak voltage of the electric pulse was simultaneously displayed on the oscilloscope.

The resistance of the patient simulator by default is $50 \Omega$. Since we want to measure the current for a range of resistances, the resistor of the simulator was replaced by power resistors of $30,50,70,80,100,120,150$ and $200 \Omega$. The peak voltage measured by the oscilloscope was used to calculate peak current using Ohm's law.

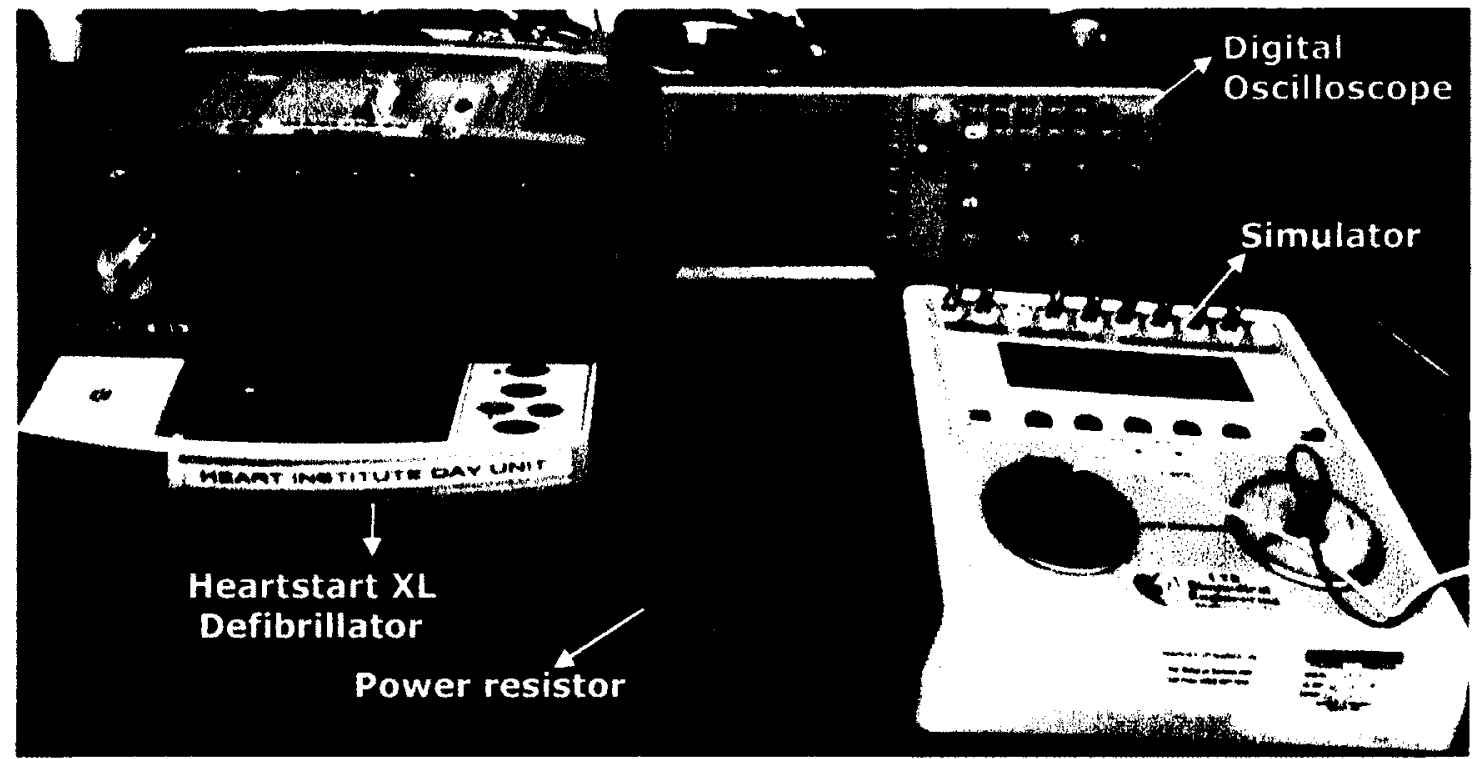

Figure 3.1 Experimental setup for measuring peak voltage.

The experimental setup is shown in Figure 3.1 and the values of peak voltages from the oscilloscope are shown in Table 3.1. 
Table 3.1 Resistance (R), Current (I), Voltage (V) and Duration ( $t$ ) of the biphasic pulse for given energy and resistance values for HeartStart XL defibrillator.

\begin{tabular}{|c|c|c|c|c|c|c|c|c|c|}
\hline XL Device & \multicolumn{3}{|c|}{$200 \mathrm{~J}$} & \multicolumn{3}{|c|}{ J 150} & \multicolumn{3}{|c|}{$100 \mathrm{~J}$} \\
\hline \multicolumn{10}{|c|}{ 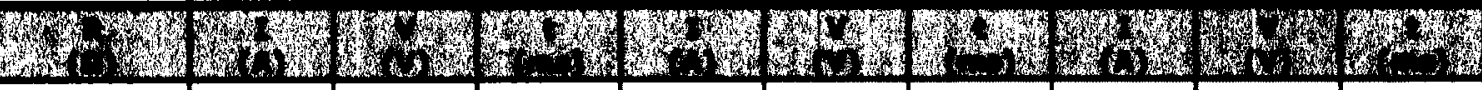 } \\
\hline 30 & 58.30 & 1750 & 6.50 & 50.00 & 1500 & 6.50 & 40.00 & 1200 & 6.50 \\
\hline & & & & & & & 3. & 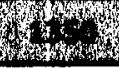 & 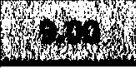 \\
\hline 70 & 27.86 & 1950 & 11.00 & 24.28 & 1700 & 11.00 & 20.00 & 1400 & 11.00 \\
\hline & & & & & & 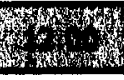 & (x) & 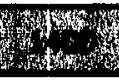 & 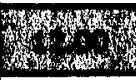 \\
\hline 100 & 20.00 & 2000 & 14.50 & 17.50 & 1750 & 14.50 & 14.20 & 1420 & 14.50 \\
\hline st & 4 & & 28 & & & 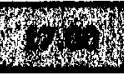 & 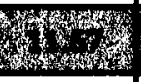 & & 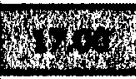 \\
\hline 150 & 13.60 & 2040 & 20.00 & 12.30 & 1850 & 20.00 & 9.87 & 1480 & 20.00 \\
\hline & & & & & & & & & Writy \\
\hline
\end{tabular}

The impedance compensating phenomena of our biphasic device is shown in Figure 3.2 . Given the same energy level $100 \mathrm{~J}$, we can see that the duration of the two waveforms are different since they represent two different resistance levels, $100 \Omega(3.3$ a) and $150 \Omega$ (3.3 b).

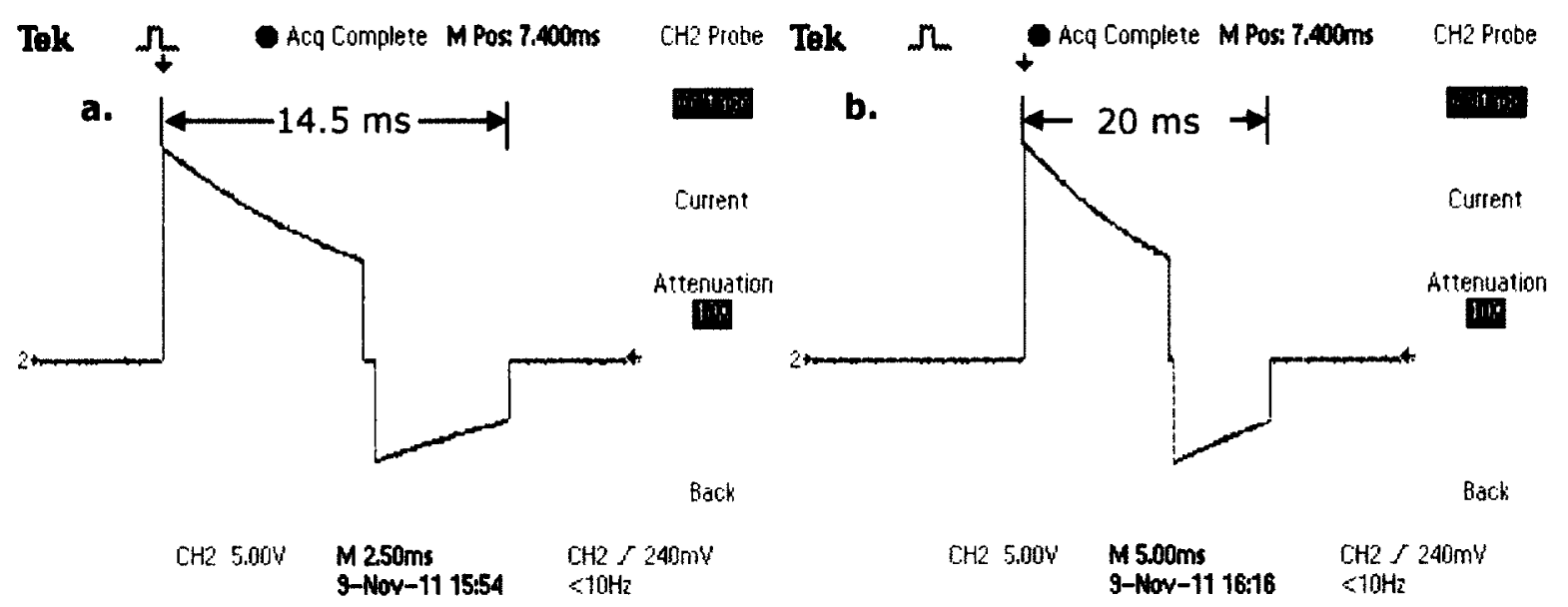

Figure 3.2 Exponential biphasic waveform for (a) 150 J, $100 \Omega$ (b) $150 \mathrm{~J}, 150 \Omega$

We also validated our experimental protocol for calculating current by repeating the same methodology using MRX defibrillator. Since $X L$ and MRX are similar devices and the MRX defibrillator gives the value of the current delivered during the operation of the device, we 
surmise that our current calculation protocol will be correct if the values we noted from the MRX device and those from $X L$ are equivalent.

From Figure 3.3, we can see that the current recorded from the oscilloscope and that recorded from the MRX device are very similar with a correlation coefficient of 0.99 . This suggests that our procedure for calculating the current using the voltage recorded from oscilloscope is correct.

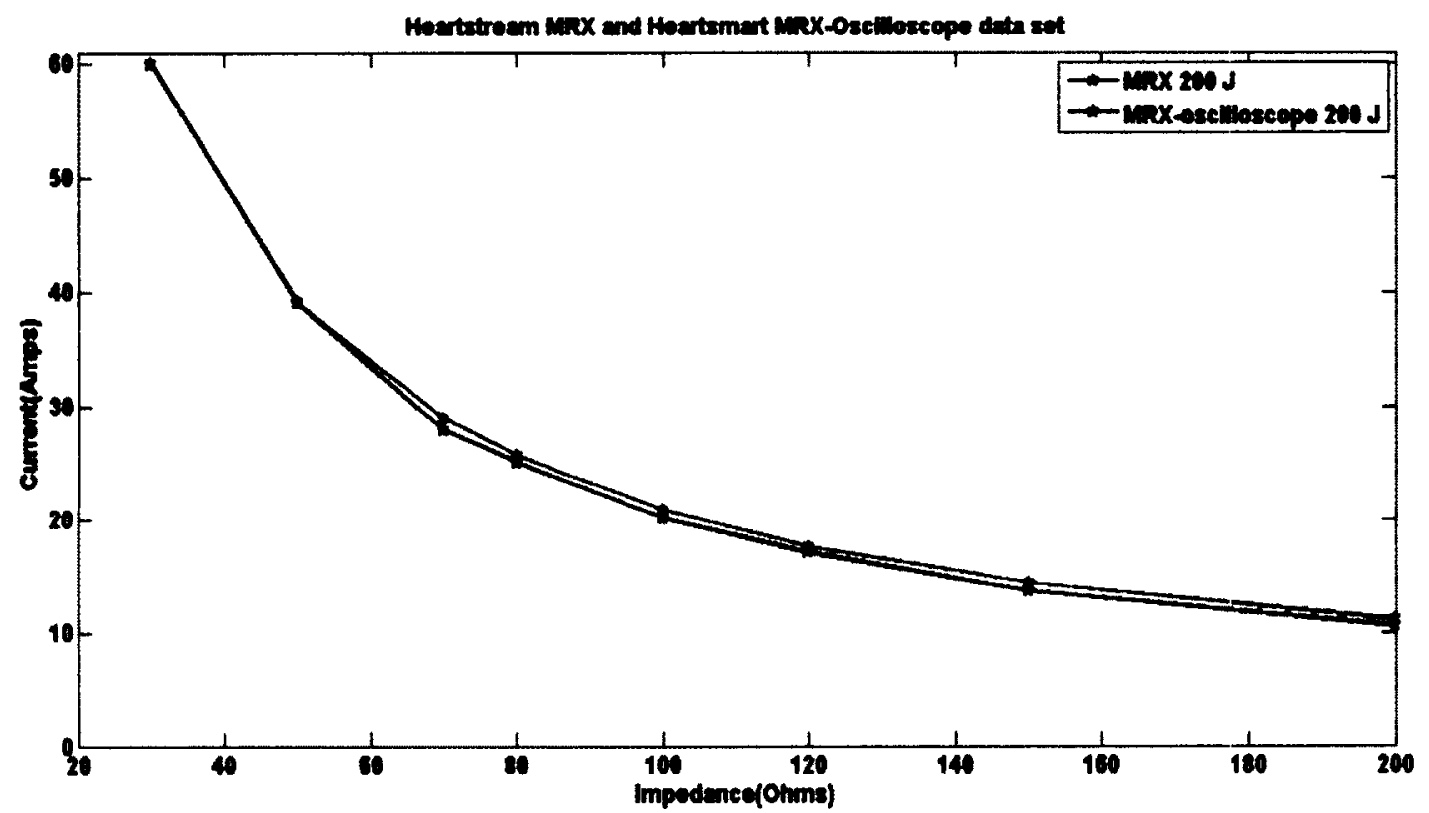

Figure 3.3 Comparison of current recorded from MRX oscilloscope and MRX device. 


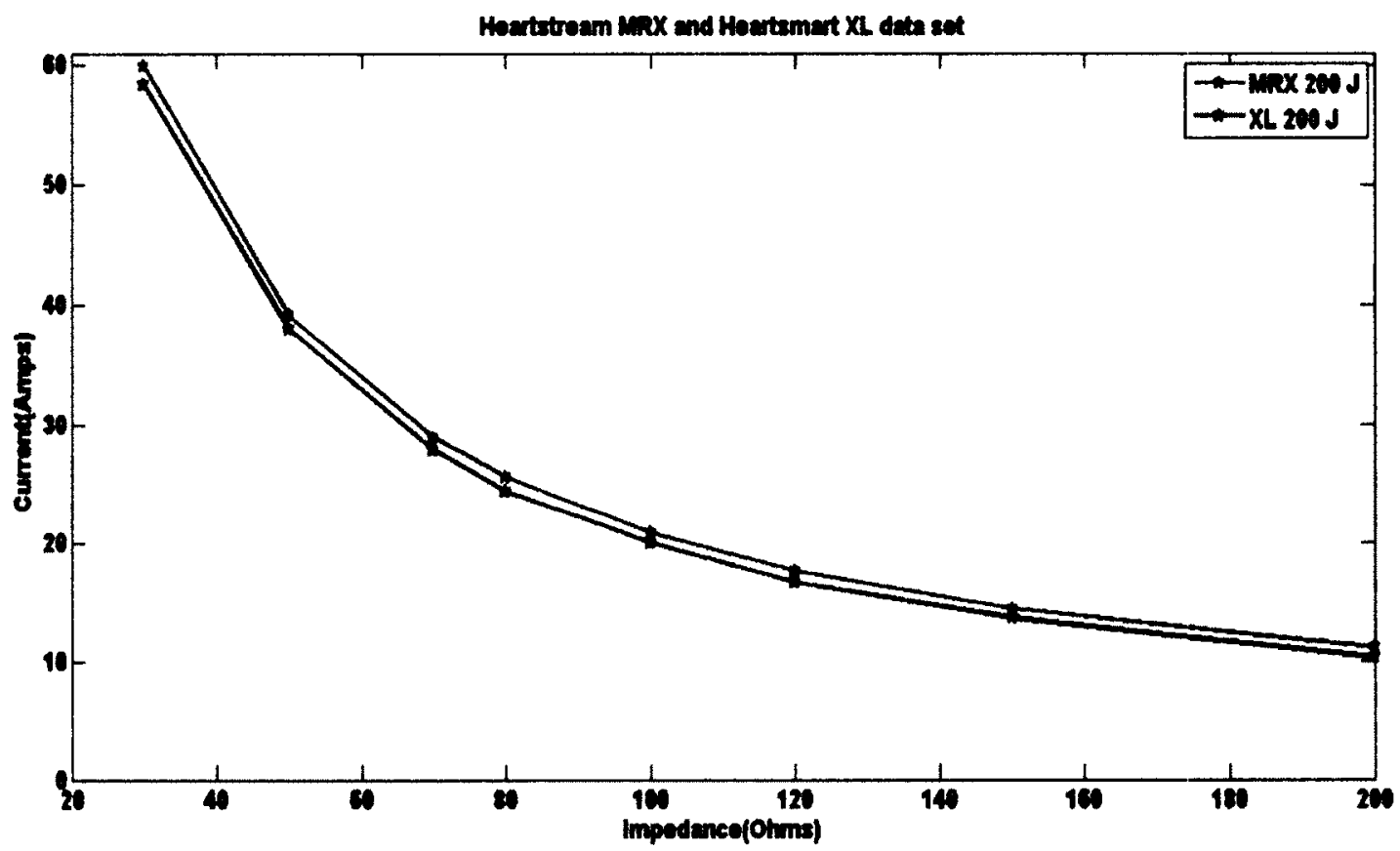

Figure 3-4 Comparison of current recorded from MRX device and XL.

Figure 3.4 shows the comparison of oscilloscope data of $\mathrm{XL}$ and $\mathrm{MRX}$ device data. Based on our assumption, there should be no deviation in the current delivered between the MRX device and $X L$ data. However, this is not the case here as seen in Figures 3.3 and 3.4. This could be the result of instrumental error induced by the oscilloscope. Nonetheless, the difference is around 2 to $3 \%$ which falls below the $10 \%$ tolerance of the oscilloscope. These results in the figure indicate that our protocol is acceptable and Table 3.1 can be used to determine the current for our data set.

\subsection{Curve Fitting}

The current delivered is measured only for a specified resistance level, as shown in Table 3.1. Given the diversity in the patient demographic, the $\pi I$ could be any continuous value which cannot all be simulated using a power resistor. Therefore, from the values of current calculated for HeartStart XL defibrillator in Table 3.1, curve fitting was performed to determine an equation and its coefficients that best fit the data points. This equation is 
needed to determine the current for any given value of TII. Given the nature of the data, power function and exponential function were tested for the best fit. The $y$ value in the equations represent current in Amperes, $x$ as impedance in $\Omega$, and $a, b$ and $c$ are constants.

The results are shown in the Figures 3.4, 3.5, and 3.6. The fit using an exponential function $y=a e^{b x}$ shows the highest root mean square error (RMSE) and sum of square error (SSE) of $2.412 \mathrm{~A}$ and $61.5 \mathrm{~A}$ respectively which suggests that the exponential function is not the best fit for our data. Power function $y=a x^{b}$ fits the data well with a RMSE of $0.568 \mathrm{~A}$; however, the function $y=a x^{b}+c$ best represents the data with the lowest RMSE and SSE of 0.39 A and $0.48 \mathrm{~A}$, respectively.

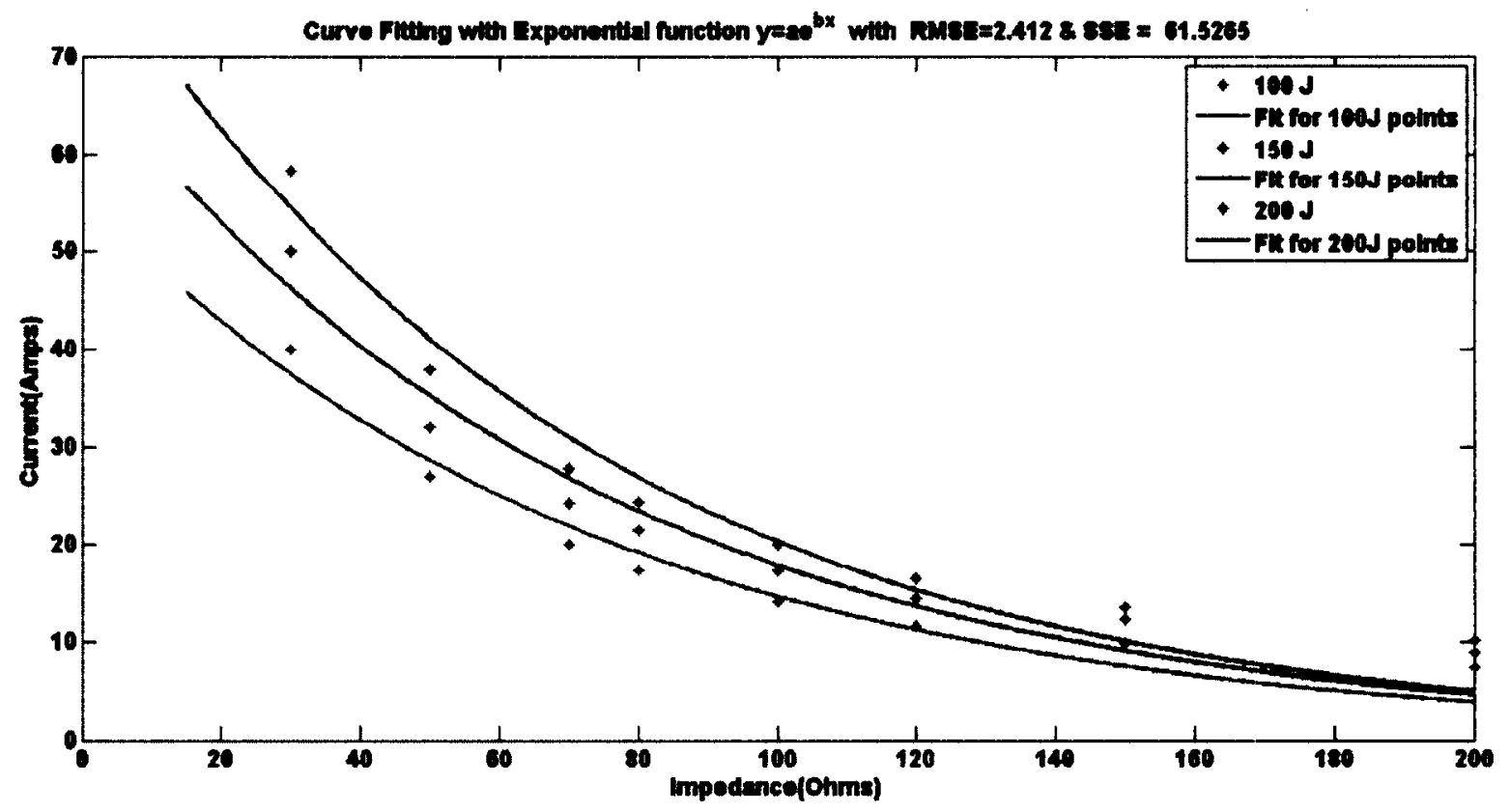

Figure 3.4 Curve fitting with exponential function $y=a e^{b x}$ for HeartStart XL defibrillator 


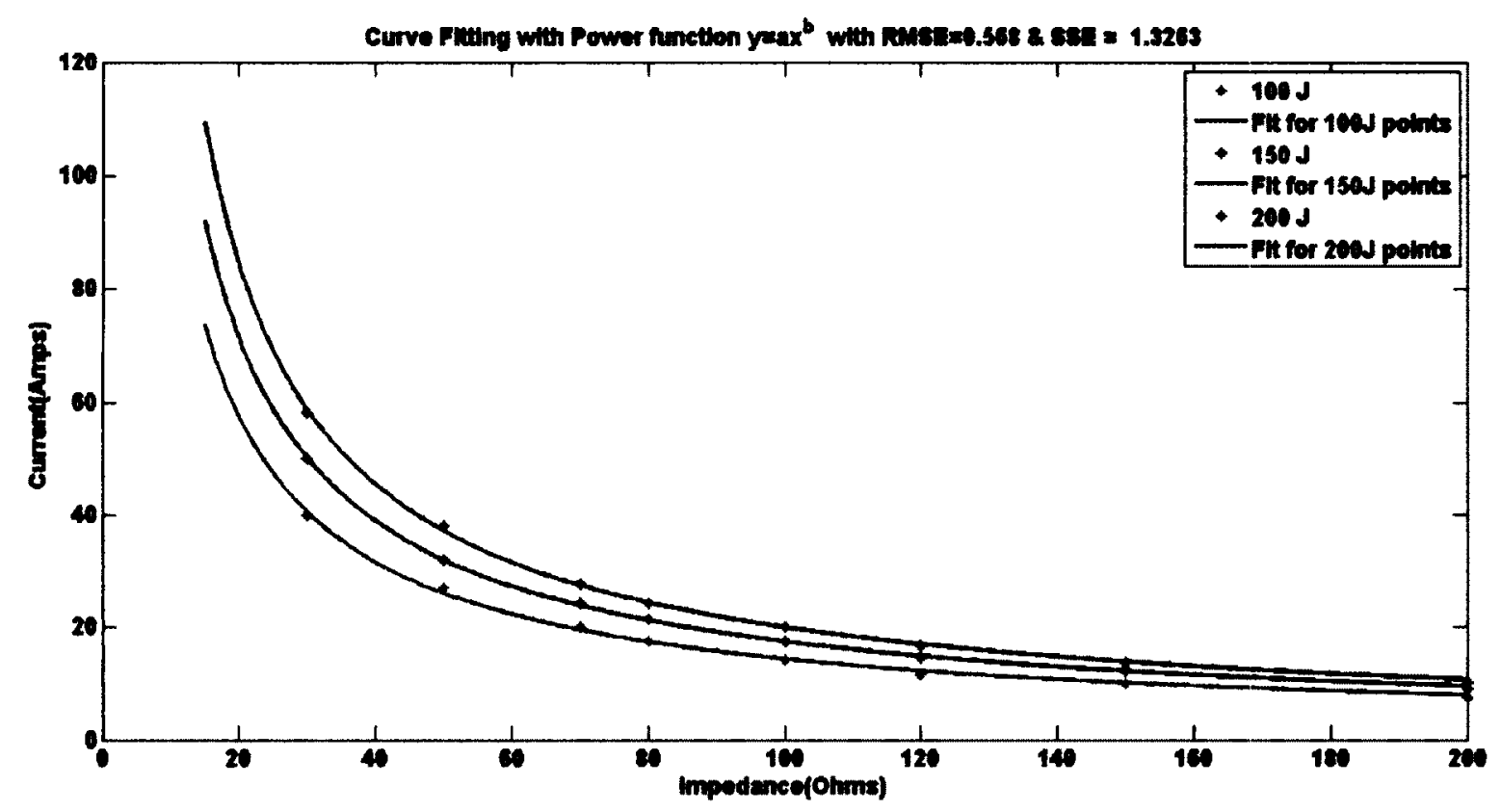

Figure 3.5 Curve fitting for power function $y=a x^{b}$ for Heartstart XL defibrillator

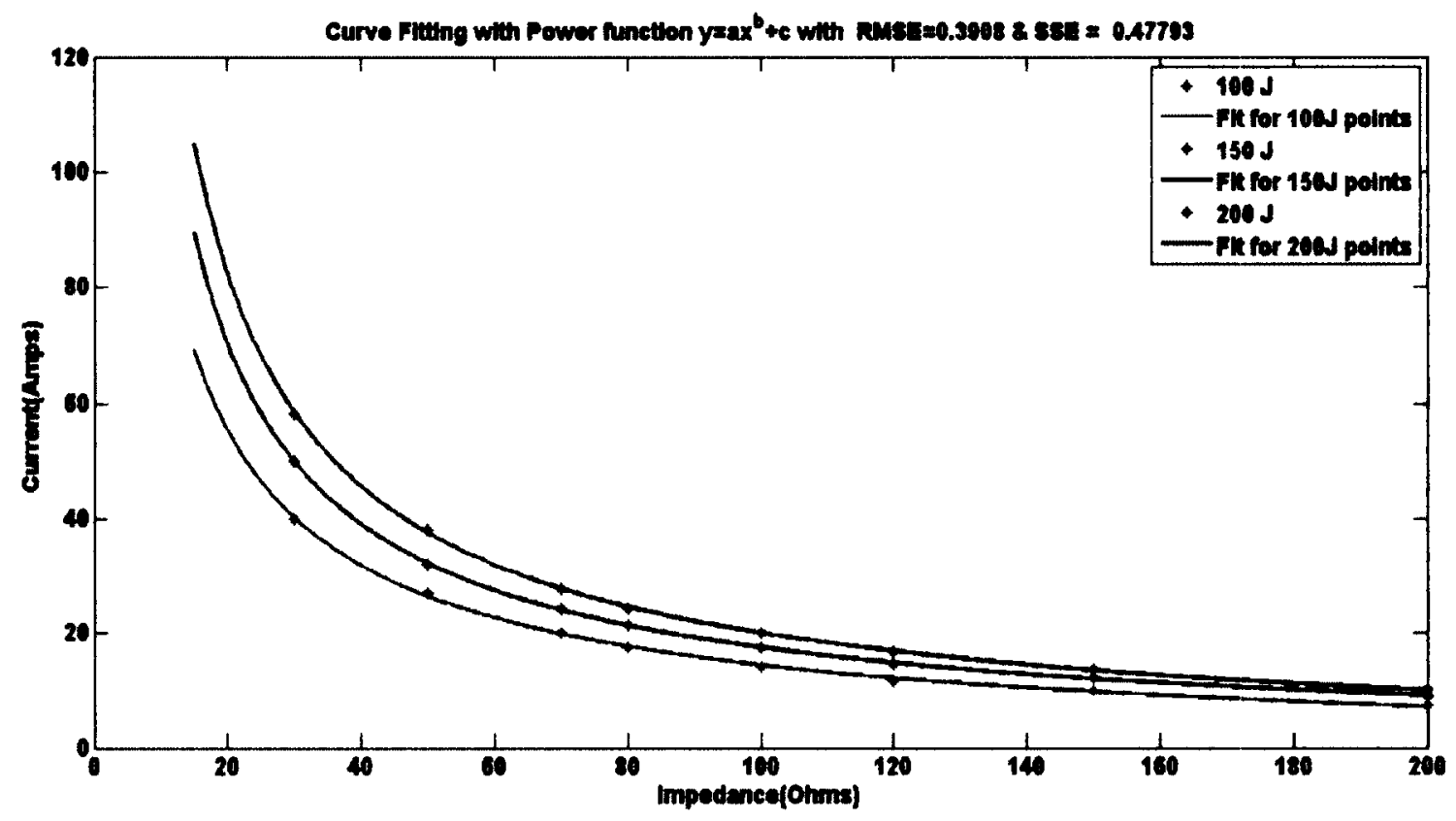

Figure 3.6 Curve fitting for power function $y=a x^{b}+c$ for HeartStart XL defibrillator 
A plausible explanation for power function best fitting the data arises from the equation,

$$
E=I^{2} R T_{\text {pulse }}
$$

where $E=$ Energy,$I=$ current, $R=$ resistance and $T_{\text {pulse }}=$ duration of the pulse.

Re-arranging (3-1) we get,

$$
\frac{E}{T_{p u l s e}}=I^{2} R
$$

Since $E / T_{\text {pulse }}=$ Power ' $P$ ', (3-2) becomes,

$$
\begin{gathered}
P=I^{2} R \\
I=\sqrt{P * R^{-1}} \\
I=P^{0.5} R^{-0.5}
\end{gathered}
$$

Since the constant $a$ and $b$ are different for each energy level, for a given energy level, $P^{0.5}$ will be constant. So, the equation now becomes analogous to,

$$
y=a x^{b}
$$

where $\mathrm{y}=I, \mathrm{a}=\mathrm{P}^{0.5}, \mathrm{x}=R$ and $\mathrm{b}=-0.5$

However, while finding the parameters $a$ and $b$, rather than $b$ being 0.5 and $a$ being square root as theoretically calculated, the power values were between 0.7 and 0.8 as seen in Table 3.2. This difference could be because the $R$ in equation 3-1 is resistance, whereas the defibrillator gives a value of impedance. This may make our equation not match the actual circuit. Also, we have assumed our $t$ to be constant, but the biphasic is impedance compensating such that the duration of the pulse $t$ changes according to the impedance, which changes the power $P$. All of this could have resulted in the power fit $y=a x^{b}$ to not be the best fit. Adding another constant $c$ to the equation could be a way to compensate for these errors, which may be the reason for third curve fitting in Figure 3.8 to yield the best fit among all the functions. 
Table 3.2 Values of the coefficients of $y=a x^{b}+c$ for 100,150 and $200 \mathrm{~J}$

\begin{tabular}{|c|c|c|c|}
\hline Energy/Coefficient & $\mathbf{a}$ & b & c \\
\hline Pr & 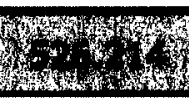 & $4+4 x^{2}$ & 7x+y \\
\hline $150 \mathrm{~J}$ & 932.264 & -0.8163 & -1.8825 \\
\hline
\end{tabular}

With these curve fitting equations, we find the current delivered for each of the case files. Our dataset now contains information about the amount of energy and current delivered in each case in addition to the type of arrhythmia, number of shocks, and the outcome of the process (Appendix-I). We then use these figures to carry out statistical analysis which is described in the next chapter. 


\section{Statistical Analysis}

This chapter investigates the clinical data mentioned in Chapter 3 using statistical tools and examines the relationship between cardioversion success and TTI.

\subsection{Method}

Between 2009 and 2011, 775 cases of cardioversion from the Day Unit, Coronary Care Unit (CCU), Cardiac Surgery Intensive Care Unit (CSICU), Nursing Units, and Reference Centre at UOHI were collected with a total of 1253 shocks for cardiac arrhythmias consisting of atrial fibrillation (AF), atrial flutter (AFL), and ventricular and supra-ventricular tachycardia (VT). The ventricular fibrillation events were excluded from the analysis. Additional details of the data can be found in Appendix-I and section 3.4 of Chapter 3.

We have classified the shocks as "successful" and "failure" based on the criteria given by the cardiologist and the Department of Biomedical Engineering at UOHI. Shocks given during cardioversion were defined as "successful" if the shock resulted in the resumption of normal sinus rhythm (NSR). In a few cases, shocks given during cardioversion produced temporary NSR followed by early return of the fibrillation wave front. These shocks were also defined as successful. When the shock did not result in restoration of NSR, they were classified as "failure". Since we are dealing with cardioversion data, patients are still alive even if the cardioversion treatment failed.

\subsection{Statistical Analysis}

We used Fischer's exact test and Chi-square test to compare the success rate of shocks with the impedance level. We chose these testing methods as both our independent (impedance) and dependent (shock success) variables are nominal/categorical variables. We used Fischer's exact test only when the number of successes or failures in any cell for a given 
analysis was less than 5 . All the statistical testing was evaluated at a significance level of $\alpha=0.05$. The null hypothesis is that there is no relationship between the independent and dependent variable.

To examine this hypothesis, we have performed statistical testing for all shocks (Table 4.1) and first shock success (Table 4.2). Testing was done separately for each of the three arrhythmias for which cardioversion was performed. These arrhythmias were categorized based on energy levels; less than or equal to $150 \mathrm{~J}(50,100$, or $150 \mathrm{~J})$ and $200 \mathrm{~J}$. We chose to divide the energy levels in to these categories because in a hospital milieu, the physicians usually start the treatment with $150 \mathrm{~J}$ energy and depending on the success/failure, the energy is increased up to $200 \mathrm{~J}$. Sometimes energy of $50 \mathrm{~J}$ and $100 \mathrm{~J}$ could also be delivered, which was few in our data set. These data were further sub-divided into two categories of high and low impedance; impedance less than or equal to $70 \Omega$ as low impedance and impedance greater than $70 \Omega$ as high impedance. We chose $70 \Omega$ as it has been reported to be average human impedance [3].

\subsection{Results}

From 1253 shocks, 952 were for AF, 125 for AFL, and 176 for VT. Table 4.1 summarizes the effect of impedance and the success rates of different selected energies for the three arrhythmias. The statistical tests show a significant relationship between impedance and success rate for AF and VT. However, for AFL the results do not indicate any influence of impedance on the shock success.

It was observed that most of the cases $(63 \%)$ were successful in the first three delivered shocks. In cases where the shock was unsuccessful, three to five numbers of repeated shocks were attempted after which the clinicians would stop the treatment even if the last shock failed in restoring NSR. 
Table 4.1: Effect of transthoracic impedance on success rates of shocks: All shocks

\begin{tabular}{|c|c|c|c|c|c|c|}
\hline gy & Arrhythmia & Impedance & $\begin{array}{c}\text { Total } \\
\text { shocks }\end{array}$ & $\begin{array}{c}\# \text { of } \\
\text { Success }\end{array}$ & $\begin{array}{c}\text { \# of } \\
\text { Failure }\end{array}$ & p-value \\
\hline & & 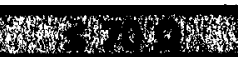 & 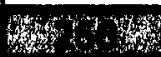 & Phath & $2 x^{2}+2$ & \multirow[b]{2}{*}{0.004} \\
\hline & 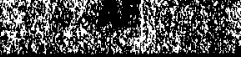 & $>70 \Omega$ & 256 & 146 & 110 & \\
\hline & & 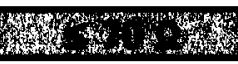 & Wres & 3. & W & \multirow{2}{*}{0.0554} \\
\hline & & $>70 \Omega$ & 52 & 47 & 5 & \\
\hline & & 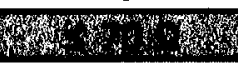 & 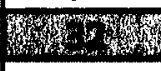 & $4 y^{4}$ & 76) & \multirow{2}{*}{$<0.0001$} \\
\hline ? & & $>70 \Omega$ & 48 & 17 & 31 & \\
\hline & & ot & m. & 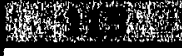 & Xit & \multirow{2}{*}{0.0103} \\
\hline$x^{2}+3 x^{2}$ & & $>70 \Omega$ & 265 & 152 & 113 & \\
\hline & & 12. & S & 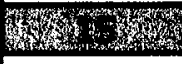 & $x^{2}$ & \multirow{2}{*}{0.1148} \\
\hline & 2. & $>70 \Omega$ & 20 & 20 & 0 & \\
\hline 8 & & $x^{n}+4$ & Hext. & (3) & 证 & \multirow{2}{*}{0.0062} \\
\hline 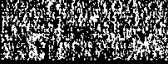 & (3) & $>70 \Omega$ & 81 & 20 & 61 & \\
\hline
\end{tabular}

As seen in Figure 4.1, the success rate is higher for low impedance patients and lower for high impedance patients for all the three arrhythmias. The treatment for VT seems to be most affected by the impedance where the success for high impedance is one-third the success for low impedance.

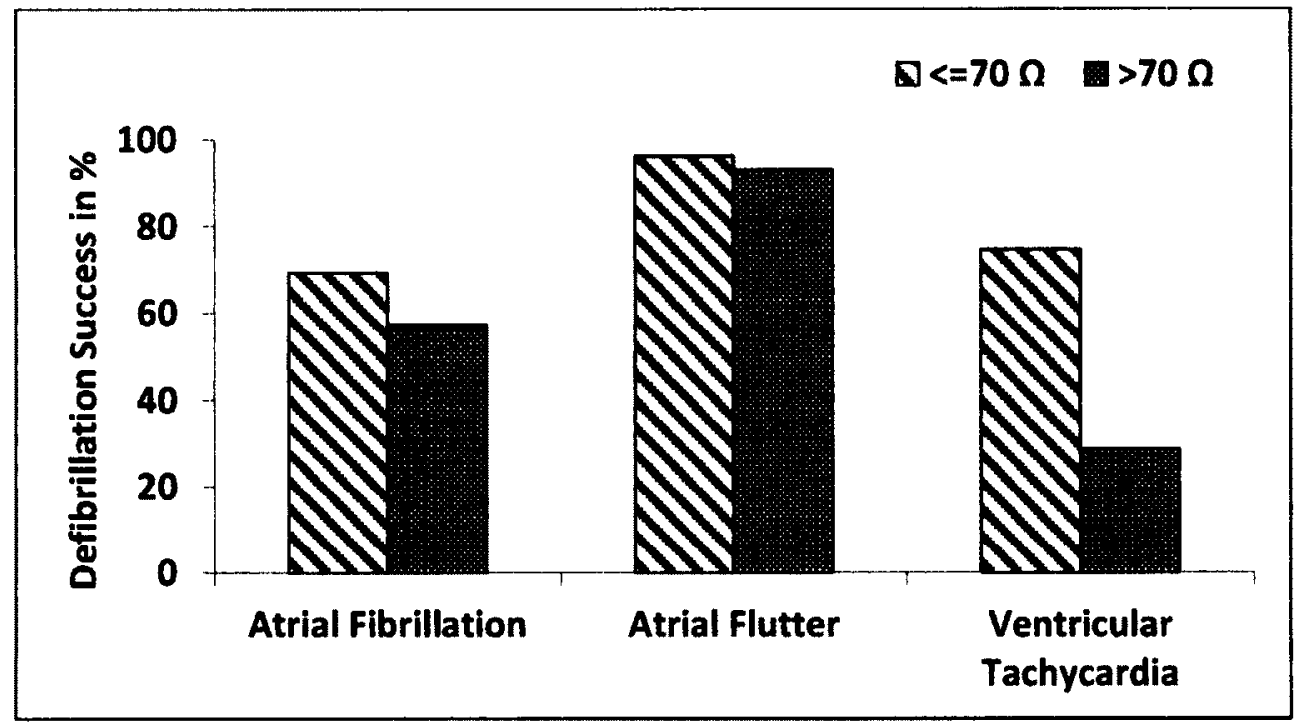

Figure 4.1 Success rate of AF, AFL, and VT for low $(<=70 \Omega)$ and high impedance $(>70 \Omega)$ : All shocks.

Table 4.2 compares the $\Pi I$ and the first shock success. The statistical results for first shock success are very similar to all shock success. There exists a significant relationship between 
impedance and first shock success for AF and VT, and AFL again shows to be unaffected by the impedance.

Table 4.2: Effect of transthoracic Impedance on success rates of shocks: First shock

\begin{tabular}{|c|c|c|c|c|c|c|}
\hline Energy & Arrhythmia & Impedance & $\begin{array}{l}\text { Total } \\
\text { shock }\end{array}$ & $\begin{array}{c}\text { \# of } \\
\text { Success }\end{array}$ & $\begin{array}{c}\text { \# of } \\
\text { Failure }\end{array}$ & p-value \\
\hline & (4) & $7+45 s$ & 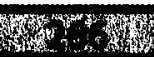 & 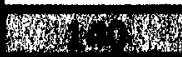 & Hors & \multirow[b]{2}{*}{0.0132} \\
\hline & $4 x^{2}$ & $>70 \Omega$ & 256 & 107 & 149 & \\
\hline & 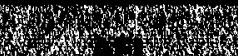 & W & $x_{3}+y_{4}$ & W & Wy & \multirow{2}{*}{0.75} \\
\hline & 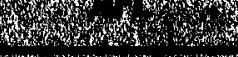 & $>70 \Omega$ & 52 & 42 & 10 & \\
\hline & (1) & $4 x^{2}$ & 6 & W & 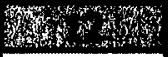 & \multirow{2}{*}{0.0058} \\
\hline y.mox & 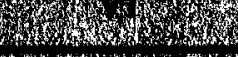 & $>70 \Omega$ & 48 & 15 & 33 & \\
\hline & (2) & H. & at & 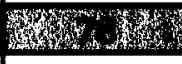 & 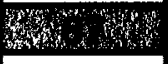 & \multirow{2}{*}{$<0.0001$} \\
\hline 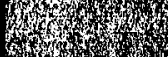 & 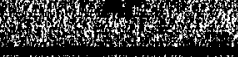 & $>70 \Omega$ & 265 & 79 & 186 & \\
\hline & - & (in & 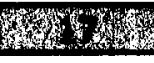 & 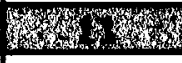 & 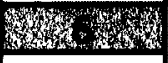 & \multirow{2}{*}{0.0625} \\
\hline & 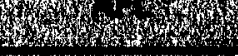 & $>70 \Omega$ & 20 & 18 & 2 & \\
\hline 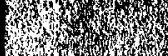 & (1) & 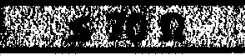 & 6es & 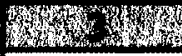 & Why & \multirow{2}{*}{0.42} \\
\hline W & 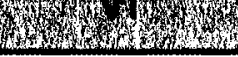 & $>70 \Omega$ & 81 & 10 & 71 & \\
\hline
\end{tabular}

Figure 4.2 also reflects on the role of impedance where its effect is more prominent for VT and AF with more success for lower impedance. From our data, we found that for a total of 952 shocks for AF, 404 shocks resulted in first shock success, and for AFL, the first shock success was the highest with 101 shocks out of 125 resulting in first shock success. It was also observed that first shock success rate was lowest for VT, where only 48 first shocks from a total of 176 shocks were successful. 


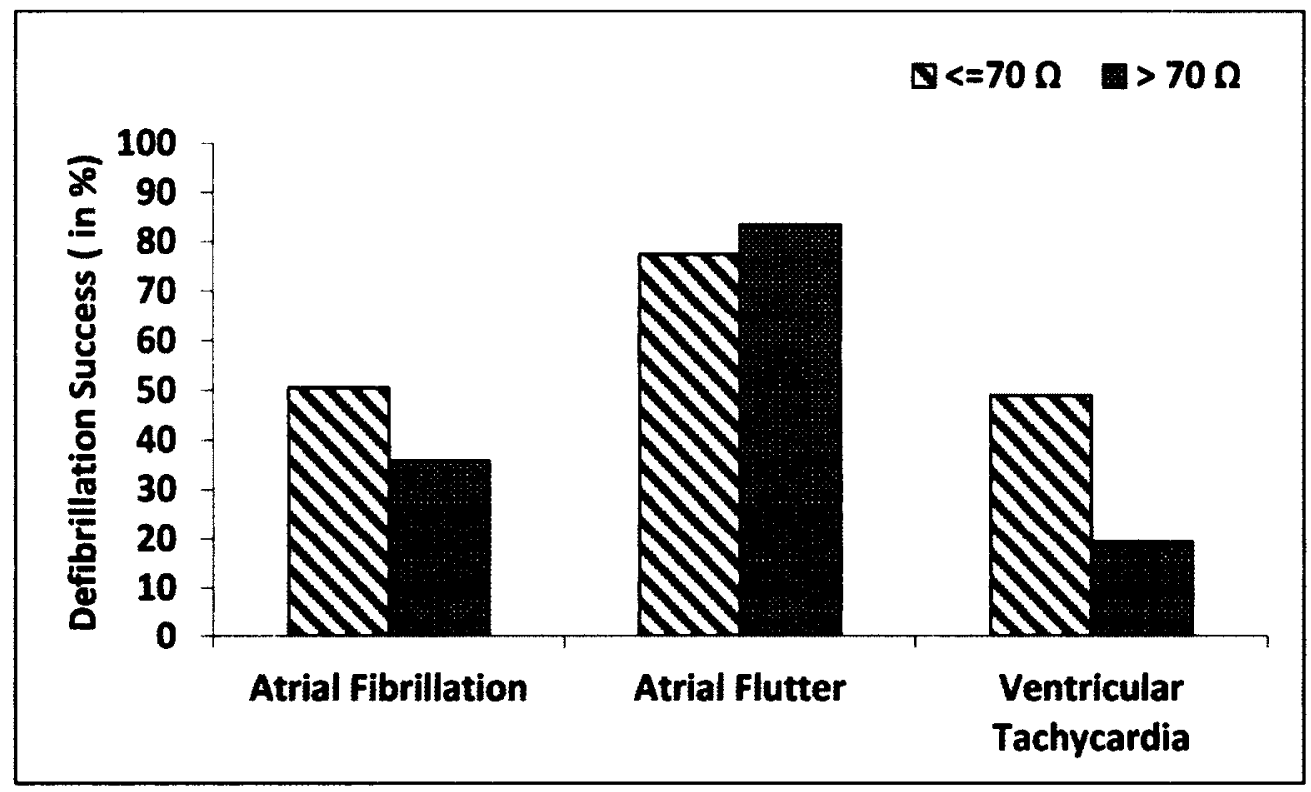

Figure 4.2 First Shock Success rate of AF, AFL, and VT for low $(<=70 \Omega)$ and high impedance $(>70 \Omega)$ : First Shock

We also compared different energy levels with the success rate for two categories of impedance, Table 4.3. The statistical results show that there is no significant difference in the success rates between the energy levels except for AFL at low impedance.

Table 4.3 : Effect of energy of the success rates for different levels of impedance: All shocks

\begin{tabular}{|c|c|c|c|c|c|c|}
\hline Impedance & Arrhythmia & Energy & $\begin{array}{l}\text { Total } \\
\text { shock }\end{array}$ & $\begin{array}{c}\text { \# of } \\
\text { Success }\end{array}$ & $\begin{array}{c}\text { \# of } \\
\text { Failure }\end{array}$ & p-value \\
\hline & & & 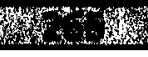 & N & 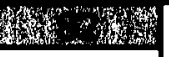 & \multirow[b]{2}{*}{0.9087} \\
\hline & & $200 \mathrm{~J}$ & 165 & 115 & 50 & \\
\hline & & - & 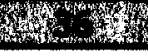 & $x$ & 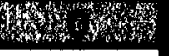 & \multirow{2}{*}{0.036} \\
\hline & 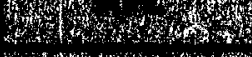 & $200 \mathrm{~J}$ & 17 & 15 & 2 & \\
\hline & & 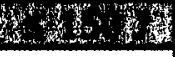 & 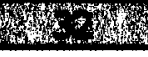 & 30 & H. & \multirow{2}{*}{0.1194} \\
\hline & 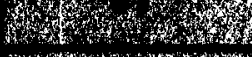 & $200 \mathrm{~J}$ & 15 & 9 & 6 & \\
\hline & & 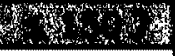 & (n) & W & 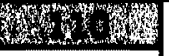 & \multirow{2}{*}{0.9398} \\
\hline & 7) & $200 \mathrm{~J}$ & 265 & 152 & 113 & \\
\hline & 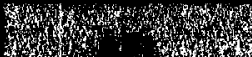 & 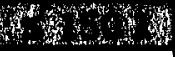 & 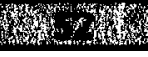 & - & - r & \multirow{2}{*}{0.1506} \\
\hline & Hets & $200 \mathrm{~J}$ & 20 & 20 & 0 & \\
\hline Why & 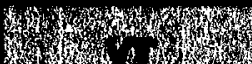 & 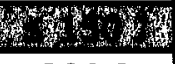 & 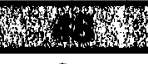 & Y & max & \multirow{2}{*}{0.193} \\
\hline 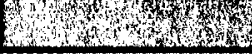 & 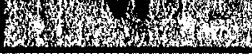 & $200 \mathrm{~J}$ & 81 & 20 & 61 & \\
\hline
\end{tabular}

The bar graph in Figure 4.3 shows the cardioversion success rate for different groups of impedance and energy. For $\mathrm{AF}$ and $\mathrm{AFL}$, the success rate appears to be independent of 
energy level. The plot for VT indicates that there is more success at lower energy level than at $200 \mathrm{~J}$ energy for both high and low impedance groups.

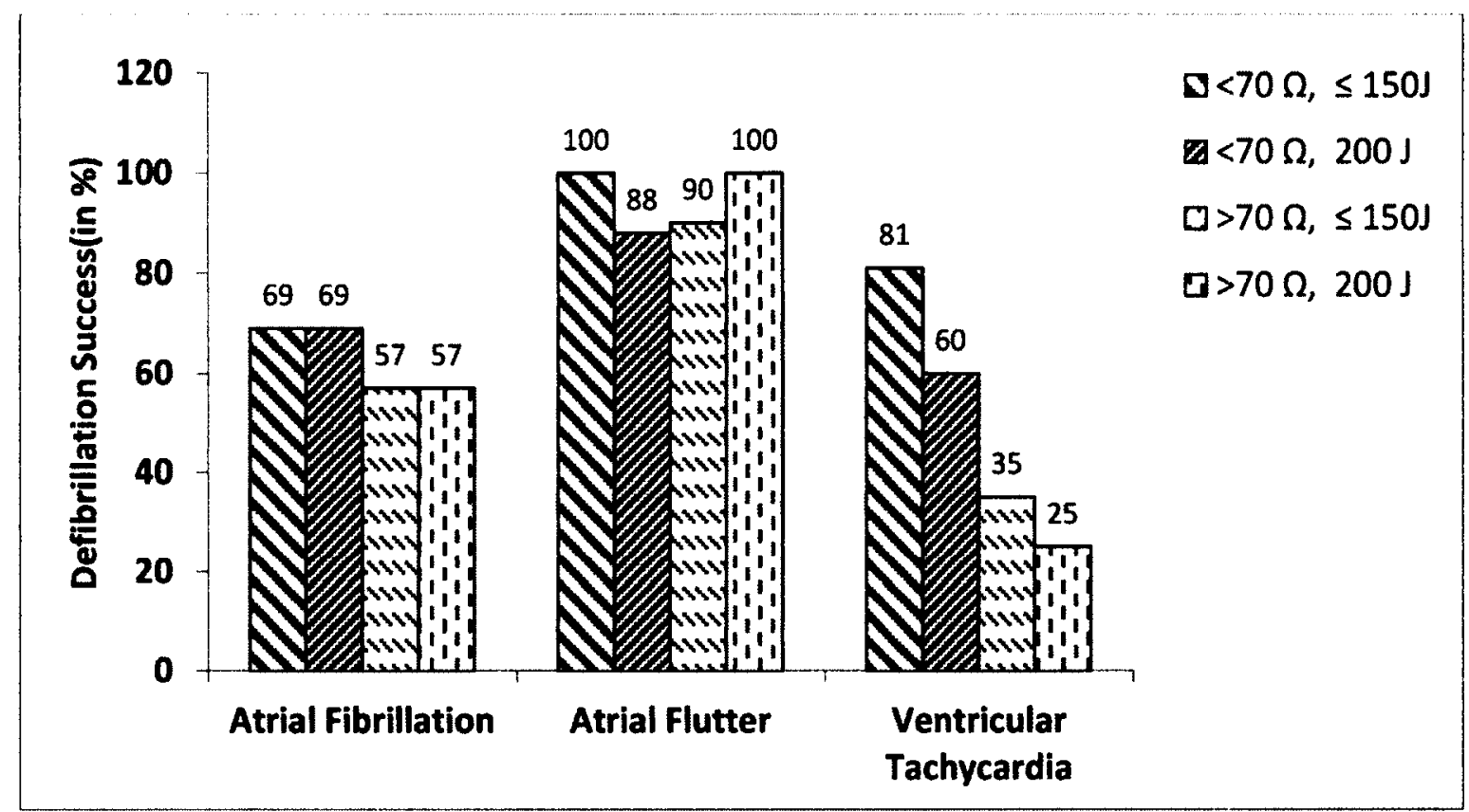

Figure 4.3 Success rates of AF, AFL, and VT for energy > $150 \mathrm{~J}$ and $200 \mathrm{~J}$ : All shocks

We plotted the cardioversion success rate against impedance for all the three arrhythmias, shown in Figure 4.4. The success rate is decreasing consistently with an increase in impedance for AF and VT. However, for AFL the success rate seems to be almost constant for different level of impedances. This plot not only helps in explaining the statistical results shown above but also indicates an inverse relationship between success rate and impedance. 

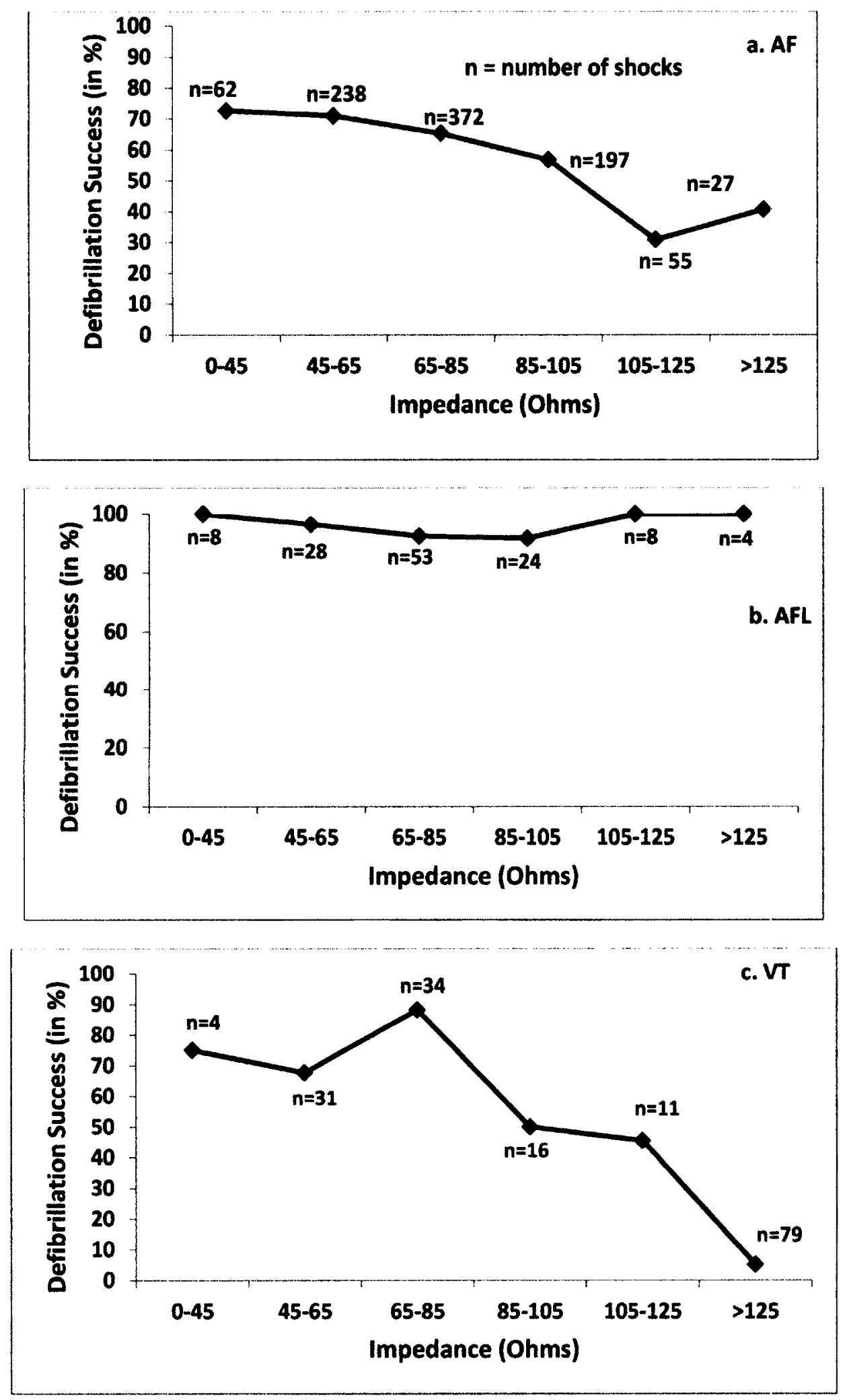

Figure 4.4 Relationship between Defibrillation success and Impedance for a. AF, b. AFL, and c. $V T$. ' $n$ ' represents number of shocks in a given impedance range. 
The relationship between peak current and shock success is shown in Figure 4.5. The relationship follows a parabolic curve for AF and VT, while almost a straight line in AFL. The curve is similar for first shock and all shock success. The different curves for the three arrhythmias indicate that each arrhythmia has its own current requirement for a successful treatment. In AF, we can observe that success for current less than $16 \mathrm{~A}$ is poor, while it starts to increase after $16 \mathrm{~A}$. The success rate between 24 to $48 \mathrm{~A}$ is constant which suggests that current between this range will lead to higher cardioversion success. Though higher current gives more success, studies have shown that giving current after a certain level induces post-shock cardiac dysfunction, leading to poor success results [70-72]. This observation holds true for our data, where we can see that for AF, success declines after 48 A. For atrial flutter, there was no clear relationship between current and shock success; high success rates were achieved at all current levels. For VT, success can be obtained between 24 to $32 \mathrm{~A}$, while current greater than $32 \mathrm{~A}$ will lower the efficacy. 

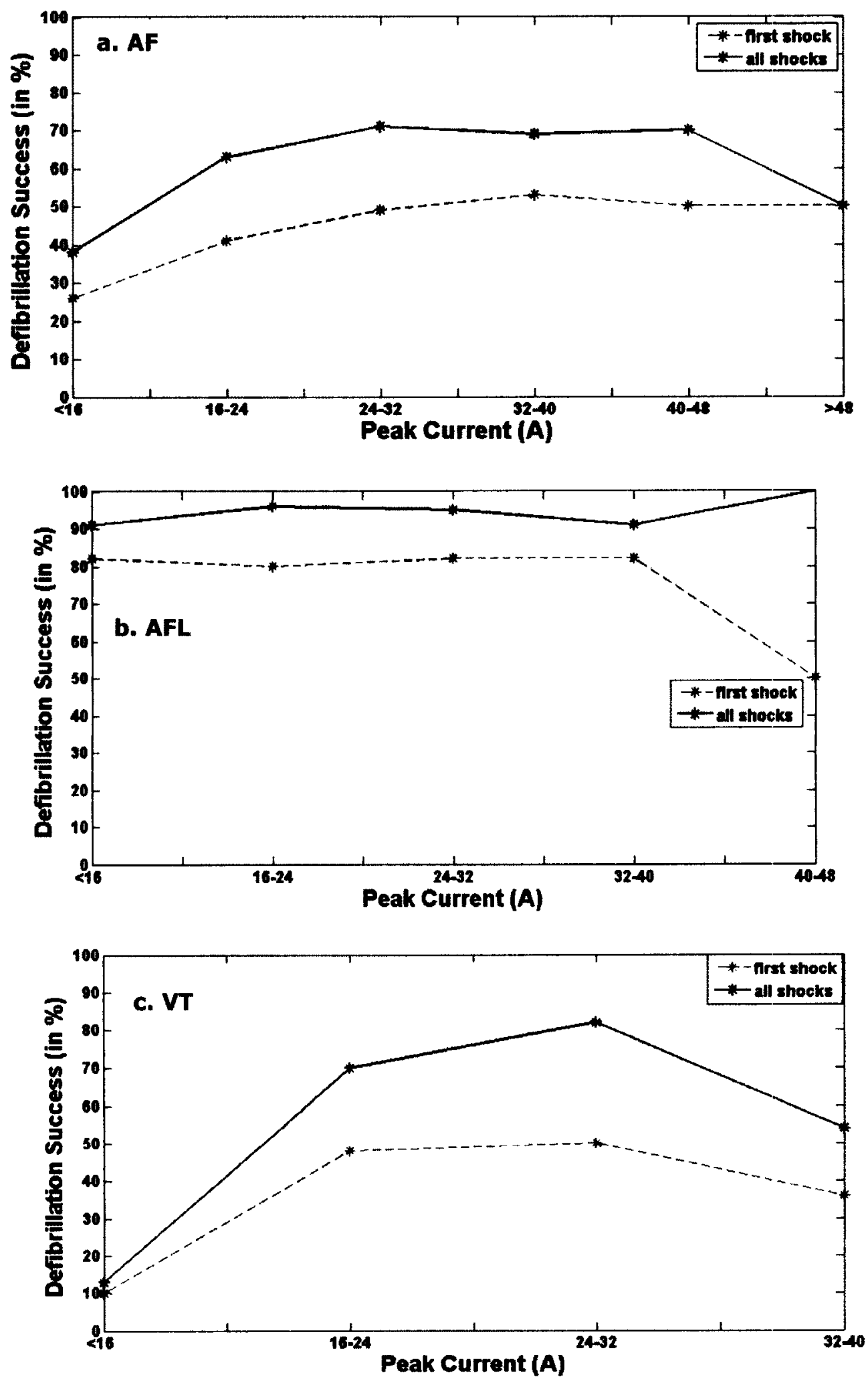

Figure 4.5 Relationship between peak current and shock success for a. AF, b. AFL, and c. VT 


\subsection{Discussion}

The results of this retrospective analysis indicate that transthoracic impedance (TTI) influences the efficacy of cardioversion when performed for arrhythmias like atrial fibrillation (AF) and ventricular tachycardia (VT). The results also show the effect of current on cardioversion success for AF and VT. The success rate for atrial flutter however is not affected by the impedance and also, the delivery of high current is not influential in increasing the success rate.

The mean thoracic impedance for our data was $80 \pm 28.5 \Omega$. However, we divided our data at $70 \Omega$ impedance, since previous studies have found this to be the average human impedance [3]. A successful defibrillation requires $95 \%$ of depolarization of fibrillating myocardial cells (atria for AF and AFL, and ventricles for VT). This is achieved by delivering sufficient amount of current to the heart. When the impedance of the patient is high, less current reaches the heart, therefore less success should be seen for such patients and this is evident from our data too. In Table 4.1 and Figure 4.1, we can notice that the success rate of high impedance patients is significantly lower for AF and VT for both high and low energy levels. Even the first shock success is higher for low impedance patients. Such low success rate for high impedance patients is likely because of delivery of insufficient current to the heart.

With the biphasic defibrillators that are impedance compensating, the results still show the effect of impedance on the success rate. This indicates that the impedance compensation technique of the biphasic defibrillators today may not be adequately effective in improving the success rate for high impedance patients. These devices compensate the energy by extending the duration of the waveform; however there is no compensation for voltage/current. Since current predicts the success of the treatment, we still see impedance 
influencing the success rate. This observation can also be made from Figure 4.6; compensating the impedance by increasing the duration after $11 \mathrm{~ms}$ does not increase voltage ( $<40 \mathrm{~V})$, therefore there will be no effective increase in the current. This observation is consistent with studies that have indicated that once the biphasic waveform extends beyond the optimal duration ( $>11.5$ to $12 \mathrm{~ms}$ ), the efficacy at that energy level decreases, while the risk of inducing post shock myocardial dysfunction increases [70-72].

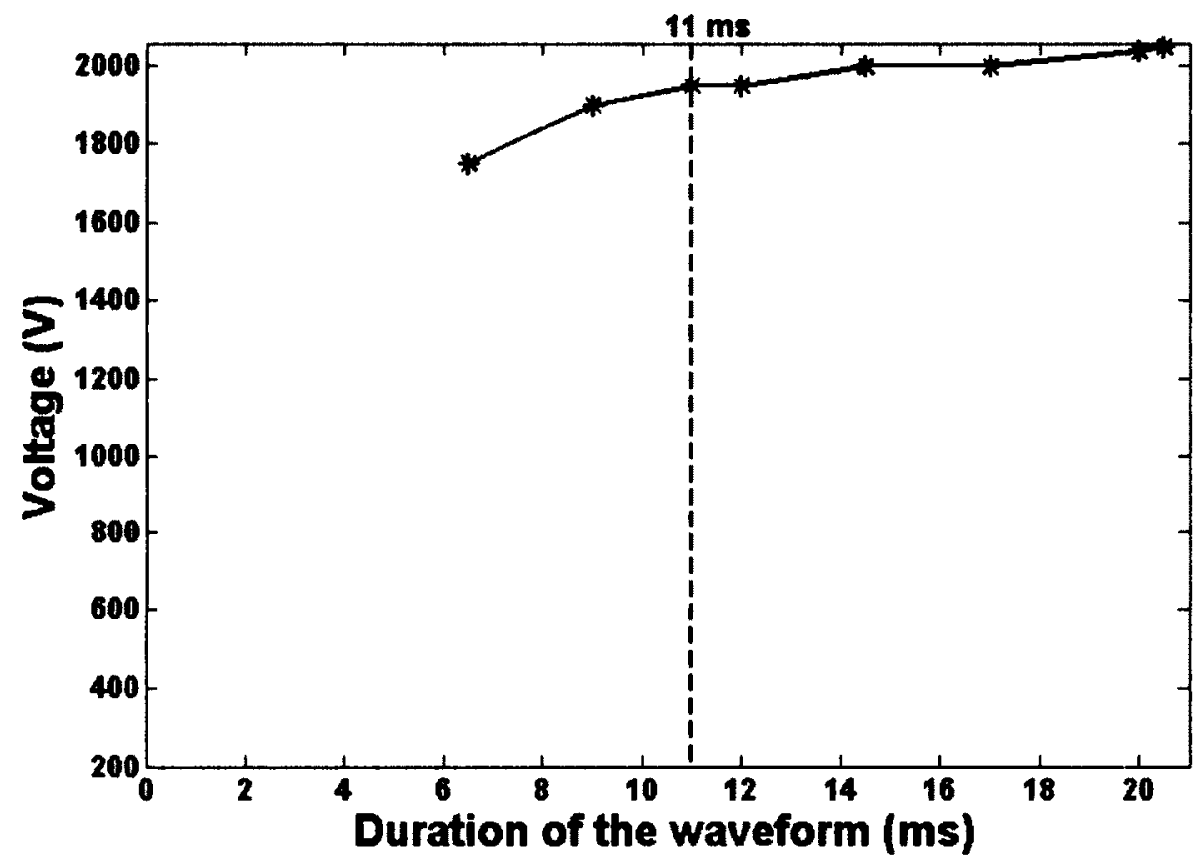

Figure 4.6 Relationship between voltage and duration of the waveform for $200 \mathrm{~J}$ energy From our current understanding of defibrillation, we expected similar shock success rate between $\leq 150 \mathrm{~J}$ and $200 \mathrm{~J}$ energy for low impedance patients and higher success rate at $200 \mathrm{~J}$ for high impedance patients. Statistical comparison between energy levels and success rate shown in Table 4.3 borne out our expectation for low impedance. However, for high impedance patients the statistical results show no difference in the success rate between high and low energy values; in fact the success rate was lowest at $200 \mathrm{~J}$ energy. This observation is surprising since increasing the energy to a high value for a given impedance results in an increase in current which ought to increase the performance of the treatment. One of the reasons for such an observation could be that the increase in current 
from one energy level may not be sufficient to defibrillate $95 \%$ of the atrial/ventricular cells. From the peak current - success relationship plot in Figure 4.5, the optimal current range for a successful cardioversion lies between 24 to $48 \mathrm{~A}$ and current greater than $48 \mathrm{~A}$ will result in damage to the heart tissue. When we compare this result with the current, voltage, and duration data mentioned in Table 3.1, we can see that for high impedance, increasing energy still results in current less than the optimal range, which may be the reason for observing low success. For low impedance, a higher energy shock may result in delivering an amount of current high enough to cause dysfunction (either persistence of fibrillation or conversion to another arrhythmia) which may have resulted in a lower success rate for $200 \mathrm{~J}$ energy. The duration of arrhythmia, pathological conditions, different pad positions, etc. could also have played a role in such an observation for the energy levels.

The overall success rate of cardioversion seems to be lowest for VT and highest for AFL. The result for AFL also indicates that impedance does not affect the performance and even the current does not impact the first shock or all shock success rate. However, these AFL results are difficult to interpret due to low statistical power resulting from lower number of failure rates. Studies done in the past show a similar result for atrial flutter, where this kind of arrhythmia has mostly yielded a success rate greater than $95 \%$ for lower energy levels [73-76]. AFL being an uncommon arrhythmia, deriving conclusions has always being difficult for this case due to less statistical power. Nonetheless, we believe that since low amplitude current is sufficient for successfully cardioverting AFL (Figure 4.5 b), we see no effect of impedance and energy on shock success. The physiological reason behind requiring less current could be due to the fibrillation wave fronts being less irregular and that they travel as a single wave in one direction in AFL as compared to the irregular contraction seen during AF/VT, making these wave fronts easily stopped even with a low current shock [14]. 
The clinical goal of cardioversion is not only to successfully defibrillate the patients but preferably to defibrillate them in the first shock. As mentioned in section 2.6.1 in chapter 2 , increasing the number of shocks have more effect in inducing cardiac damage and exacerbating the arrhythmia than in increasing the chances of success. Our data showed $42 \%$ first shock resulted in success for AF, $81 \%$ for AFL, and $27 \%$ for VT. It was also noted that the first shock success rate was not only dependent on cardiac arrhythmias but aiso the TII where the success rate for patients with lower TII was higher than patients with high TTI.

\subsection{Conclusion}

The Chi-Square and Fischer's tests used for statistical analysis show the effect of impedance on the success rate of cardioversion with the effect being arrhythmia specific. While for VT and AF the impedance largely affected the success, no change was observed in the success rate for AFL at the two impedance levels.

With the impedance compensating biphasic defibrillator technology, the effect of impedance on a shock success still persists. This is largely because the impedance compensation is done for energy and not for current delivered and it is the current that results in the defibrillation of the heart.

Our results show that giving high current ( $>48 \mathrm{~A}$ for AF and $>40 \mathrm{~A}$ for VT) does not necessarily increase the efficacy. This high level current is seen to be delivered for impedance less than $50 \Omega$. Since in clinical practice, the impedance of the patient is unknown during the process, there could be a chance that defibrillating such patients even with $100 \mathrm{~J}$ of energy would induce post-shock cardiac dysfunction. Therefore, in order to maximize the results of cardioversion, it is important that clinicians take measures to minimize the effect of TTI. This could be done in number of ways $i$. presenting the 
information of TII to the clinicians so that they can select the energy level accordingly, ii. changing the pad position and also the electrode type (e.g. hand held paddles) iii. reducing the skin-impedance by altering the skin-prep method etc. iii. applying pressure to the thorax to reduce the air volume in the lungs that contributes to TTI.

\subsection{Limitations}

There are several issues that might limit the generalizability of the conclusions. One of the limitations of this analysis is the use of "treatment" as an independent variable. The choice of therapy is largely driven by the clinical assessment of the patient and the cardiologist i.e. treatment is dependent on the clinical setting. This causes inclusion of the effect of such confounding variables in the data which makes it difficult to interpret the results accurately.

Along with current delivered, another vital parameter that has been shown to influence the success rate is the duration of the occurrence of the arrhythmias [1][2]. One of the major limitations of this study is the exclusion of this parameter for the analysis. Since, it is a retrospective analysis, obtaining these data were difficult and including this information would necessitate a significantly longer study.

Other factors like medications and patient's pathological condition have not been considered in this study. The collection of this information was beyond the scope of this study. However, this information is unlikely to change the results obtained in a measurable way because the data collected already contains important determinants for cardioversion identified by other researchers, like energy, current and impedance [4].

Another potential limitation of this study involves evaluating data from only one manufacturer. The biphasic defibrillators available today vary in their electrical characteristics like peak voltage, waveform shape and impedance compensation method 
between manufacturers. Further research is indeed required for the generalisation of the results. However, research done in the past has shown Philips HeartStart XL and HeartStart MRX to yield better performance than other available biphasic defibrillators [77].

Despite these weaknesses, the major strength of this study is that it is the first to examine the role of TTI on the success rate of biphasic defibrillators for different levels of impedance and energies. Our work has attempted to clarify the controversy regarding the effect of TTI for impedance compensating biphasic defibrillators where the shock success appears to be inversely related to the patient's TII. The statistical results are reliable because of the large dataset we have used to perform our analysis ( 775 cases and 1253 shocks). This suggests the information provided by our study can be generalized to cardioversion treatment carried out in other patients. 


\section{Finite Element Method}

\subsection{Introduction}

The statistical analysis in the previous chapter suggests the influence of $\Pi 1$ on a successful cardioversion; however, it is insufficient in explaining the effect of impedance on the current reaching the heart. One key to understanding the effect of $\pi I$ is to uncover the current distribution in the thorax and the levels of current that actually reach the myocardium during defibrillation. This current density is affected by inhomogeneous tissues that are present in the thorax. Therefore, comprehension of the distribution of the current through different tissue types in the thorax is important. This understanding may aid in interpreting the observations noted in Chapter 4, and may also help formulate new strategies for a "first-shock" successful defibrillation.

One practical way to investigate the electrical activity in the thorax is to construct a model that represents the human thorax as accurately as possible. It is known that electric signals in the body can be described by a solution to a quasi-static Laplace equation. Computationally solving this equation on a human thorax model will foster an insight on the current distribution during defibrillation.

Computational modelling removes the need for invasive measurements of the electrical activity inside the body. It also allows researchers to perform different experiments not feasible in living tissues. These advantages have contributed to the application of numerical modelling in the medical field, aiding in development of new diagnostic and therapeutic tools based on physiological function. 
Various techniques have been used to solve the governing Laplace equation; the common methods being the Finite Element Method (FEM), the Boundary Element Method (BEM), the Finite Difference Method (FDM), the Moments Method (MM), and the Monte Carlo Method (MCM). FDM, MM and MCM are adequate for linear problems with regular geometry, while BEM is limited to solving only the boundary value problems. FEM has been found to be appropriate for solving both linear and non-linear problems on any arbitrarily shaped domains. Given the complex shape and inhomogeneity of the thorax, FEM has been extensively used for solving thoracic potential and current during defibrillation [51], [52], [55-66]. With the help of geometric information extracted from a high resolution image, accurate thoracic models incorporating various inhomogeneous regions can be constructed using FEM.

\subsection{Problem Formulation}

In electrophysiology, the human body is described by a volume conductor. For a volume conductor, the relation between the electric field $\vec{E}$ and the magnetic field $\vec{B}$ is given by Maxwell's equation:

$$
\nabla \times \vec{E}+\frac{\partial \vec{B}}{\partial t}=\mathbf{0}
$$

For the frequency and conductance values associated with the heart tissue, time varying electric and magnetic fields can be neglected and at any instant, the fields can be treated as stationary. That is,

$$
\frac{\partial \vec{B}}{\partial t}=\mathbf{0}
$$

So (5-1) can be reduced to,

$$
\nabla \times \vec{E}=\mathbf{0}
$$


Since the electric field is irrotational, $E$ can be expressed as the gradient of a scalar field $u$

$$
\vec{E}=-\nabla \boldsymbol{u}
$$

In a conductive domain, the current density $\vec{J}$ is related the electric field $\vec{E}$ by Ohm's law,

$$
\vec{J}=\sigma \vec{E}
$$

Substituting (5-4) into (5-5), we get

$$
\vec{\jmath}=\sigma(-\nabla \boldsymbol{u})
$$

Assuming that there are no current sources or sinks in the domain and only an external source drives the current, applying conservation of charge yields,

$$
\nabla \cdot \vec{J}=0
$$

Taking divergence on both sides for (5-6) and comparing with (5-7), we get

$$
\boldsymbol{\nabla} \cdot(\boldsymbol{\sigma} \boldsymbol{u})=\mathbf{0} \text { in } \boldsymbol{\Omega}
$$

(5-8) is an elliptical partial derivative equation known as the Laplace equation. This Laplacian describes the relationship between conductivity and the potential distribution of the domain $\Omega$ and is valid only when the object inside the domain has linear and isotropic conductivity.

The potential distribution inside a domain $\Omega$ is a result of injected current density in the boundary of the domain $r$. Boundary conditions are therefore imposed on the model to represent this excitation current applied to the electrodes present on the boundary. In our work, we consider mixed Neumann and Dirichlet boundary conditions. The Neumann boundary represents the conservation of charge condition defined by,

$$
\sigma \frac{\partial u}{\partial \hat{n}}= \begin{cases}J & \text { electrodes } \\ 0 & \text { elsewhere }\end{cases}
$$


Equation 5-9 implies that inward normal component of the current density $\left(\sigma \frac{\partial u}{\partial \hat{n}}\right)$ is zero everywhere except at the places where the electrodes are attached. The Dirichlet boundary condition is applied to obtain reference condition for the potential. This is done by grounding one of the boundary electrodes as shown below,

$$
u=0 \text { on } \Gamma_{1}
$$

Here potential $u$ on the region in the boundary underneath one of the electrode $\Gamma_{1}$ is set to zero. This Dirichlet boundary condition can be alternatively applied by randomly selecting a node in the model and forcing it to zero.

Imposing the above boundary condition, (5-8) is numerically solved for the potential distribution in the heart using FEM.

\subsection{Computational Modeling using FEM}

The solution of (5-8) using FEM follows the following steps:

1. Discretizing the domain:

The first step in FEM is to discretize the domain $\Omega$ into finite non-uniform, nonoverlapping elements connected to each other by nodes. The elements can be triangle or rectangle in 2-D and tetrahedron or hexahedron in 3-D. In this thesis, we use simplices, i.e., triangles for 2-D and tetrahedron for 3-D, to represent the finite element mesh. The finite element mesh is usually described as several arrays containing information about the nodal co-ordinates, nodes representing the element and element conductivities. 


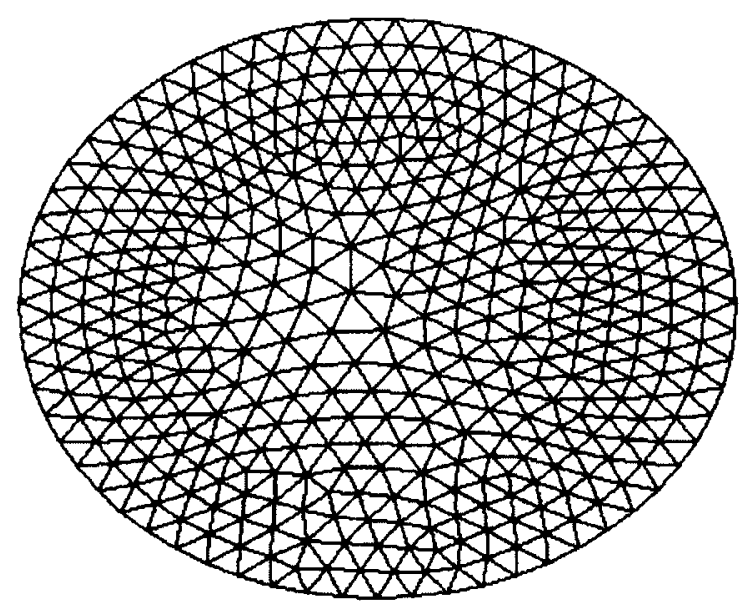

a)

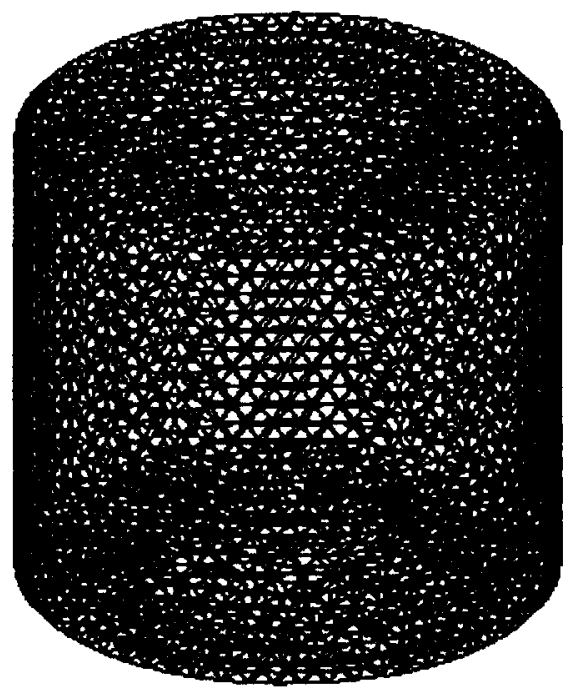

b)

Flgure 5.1 Example of FEM discretization a) 2-D mesh with 467 elements and b) 3-D mesh with 37874 elements

2. Selecting the interpolation functions:

The potential to be solved is approximated within each element using an interpolation function defined by the values of the potential on the nodes representing that element. For this work, a linear interpolation function is used for simplicity.

3. Formulation of element matrices:

This step involves calculating local stiffness matrices for each element (details in section 5.4).

4. Assembling local matrices to obtain overall system equation:

Here, the element matrices are assembled to form a system matrix denoted by $Y$. The final assembled equation is written in a matrix form, $\boldsymbol{V}=\mathbf{Y Q}$ with $V$ representing vector of nodal potentials, $Y$ as system of matrix and $Q$ as vector of input current. This equation is then modified to incorporate the boundary conditions. 
5. Solution of the overall system of equations:

The last step includes solving the finite element global equation system which is solved using MATLAB R2010a. The mathematical descriptions are mentioned in section 5.4 .

6. Calculation of the potential and current density of each element:

The solution of the system equation yields the nodal potentials. Using these values, the potential gradient in each of the elements is determined using linear interpolation. This potential when multiplied with the conductivity, gives the current distribution in each of the element.

The next section describes the mathematical approach based on the step-by-step procedure described above. The finite element formulations are heavily borrowed from [78] .

\subsection{Numerical Methods}

For 3-D FEM analysis, the first step involved discretizing the domain $\Omega$ into a 3-D mesh consisting of $N_{e}$ tetrahedral elements formed by $N_{n}$ nodes. The FEM mesh is generated using NETGEN v.4.9.13 [79] using the EIDORS version 3.5 [80]. Each element is represented by $N_{D+1}$ number of nodes, $N_{D}$ being the dimension of the model ( 3 for $3-D$, and 2 for $2-D$ ). The conductivity $\sigma_{e}$ is assigned to each element and it is assumed constant over the element. Along the boundary of the model $\Gamma$, two electrodes are attached, each of which are represented by multiple nodes. The position of the nodes are denoted by $n_{p}=\left[n_{x}, n_{y}, n_{z}\right]$ where $n_{x}, n_{y}, n_{z}$ are the $x, y$, and $z$ co-ordinates of the nodes, respectively.

The next step after domain discretization involves the calculation of local matrix of each element $S_{e}$ defined as,

$$
S_{e}=\frac{2 \sigma_{e} B^{T} B}{N_{D} !|\operatorname{det} A|}
$$


where, $A$ is defined as,

$$
A=\left[\begin{array}{llll}
1 & n_{x 1}^{1} & n_{y 1}^{1} & n_{z 1}^{1} \\
1 & n_{x 2}^{2} & n_{y 2}^{2} & n_{z 2}^{2} \\
1 & n_{x 3}^{3} & n_{y 3}^{3} & n_{z 3}^{3} \\
1 & n_{x 4}^{4} & n_{y 4}^{4} & n_{z 4}^{4}
\end{array}\right]^{-1}
$$

Matrix $B$ is the sub matrix of $A$ with the top row deleted.

The local matrix $S_{e}$ is then assembled to form a system matrix $Y$ which is,

$$
Y=C^{T} S C
$$

where $\mathrm{C}$ is the connectivity matrix associating global nodes $j$ to local nodes $i$ of each element,

$$
C_{i j}= \begin{cases}1 \text { if } \text { global node } i \text { corresponds to local node } j \\ 0 \quad \text { elsewhere }\end{cases}
$$

and $S$ is block diagonal of local matrix of all the elements defined as,

$$
S=\left[\begin{array}{cccc}
S_{1} & 0 & \cdots & 0 \\
0 & S_{2} & \cdots & 0 \\
\vdots & \vdots & \ddots & \vdots \\
0 & 0 & \cdots & S_{N_{e}}
\end{array}\right]
$$

The equation to represent the behaviour of the domain is described by Ohm's law which is represented in a matrix form as,

$$
V=Y^{-1} Q
$$

where $\mathbf{V}$ is vector of nodal potentials, $\mathbf{Y}$ is the system matrix and $\mathbf{Q}$ is the vector of current inputs to each node (with Neumann Boundary condition).

Inversion of $Y$ is required to solve (5-13). Therefore, a reference node is selected so that $Y$ becomes a non-singular matrix. This is done by selecting a node and setting the voltage on that node to 0 . 


\subsection{Imposing Boundary Condition}

Under realistic conditions, electrodes on the skin have associated skin-electrode contact impedances. To incorporate this impedance in our model, Complete Electrode Model (CEM) is implemented which takes into account the effect that the contact impedance $z_{c}$ has on the electrical potential of the domain.

The measured potential on the boundary now becomes,

$$
u+z_{c} \sigma \frac{\partial u}{\partial n}=U_{l} \quad \text { on } E_{l}, l=1,2, \ldots \ldots, L
$$

Here, $z_{c}$ is the contact impedance, $l$ is the electrode number, $U_{l}$ is the constant potential on the electrode $l$. The outward normal term $\frac{\partial u}{\partial n}$ denotes that the contact impedance is only a factor for current passing across the electrode-skin boundary $d \Gamma$.

The boundary condition (5-9) is implemented in CEM by taking the integral of (5-9) where the integral of current density over the surface of each electrode must equal the applied current,

$$
\int_{E_{l}} \sigma \frac{\partial u}{\partial n} d \Gamma=I_{l}, \text { for } l=1,2, \ldots \ldots, L
$$

where, $l_{l}$ is the current sent to the $I^{\text {th }}$ electrode and $E_{l}$ denotes the region on the boundary $\Gamma$ that corresponds to the $f^{\text {th }}$ electrode. In the inter-electrode gap where there is no electrode, the following boundary condition is applied.

$$
\sigma \frac{\partial u}{\partial n}=0 \text { on } d \Gamma \backslash \bigcup_{l=1}^{L} E_{l}
$$




\subsection{Derivation of Linear Interpolation function ' $\phi$ '}

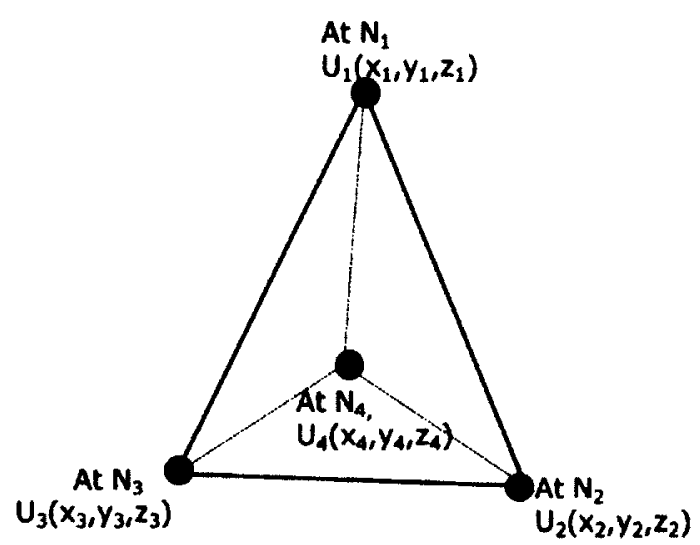

Figure 5.2 A tetrahedral element

Using the concepts of Barycentric co-ordinates, the potential within a tetrahedral element (Figure 5.2) can be approximated by the linear function as,

$$
U(x, y, z)=a+b x+c y+d z=\left[\begin{array}{llll}
1 & x & y & z
\end{array}\right]\left[\begin{array}{l}
a \\
b \\
c \\
d
\end{array}\right]
$$

The above equation must hold true for each node where $U=U_{i}$ when $(x, y, z)=\left(x_{i}, y_{i}, z_{i}\right)$. The coefficients $a, b, c, d$ can now be found using four independent simultaneous equations formed from the four nodes $N_{1}, N_{2}, N_{3}$ and $N_{4}$ representing the element. Assuming that the values of the potential at the nodes are $U_{1}, U_{2}, U_{3}, U_{4},(5-17)$ can be written as,

$$
\left[\begin{array}{l}
U_{1} \\
U_{2} \\
U_{3} \\
U_{4}
\end{array}\right]=\left[\begin{array}{llll}
1 & x_{1} & y_{1} & z_{1} \\
1 & x_{2} & y_{2} & z_{2} \\
1 & x_{3} & y_{3} & z_{3} \\
1 & x_{3} & y_{4} & z_{4}
\end{array}\right]\left[\begin{array}{l}
a_{1} \\
b \\
c \\
d
\end{array}\right]
$$

Using inverse matrix, the coefficients $a, b, c, d$ are determined by

$$
\left[\begin{array}{l}
a_{1} \\
b \\
c \\
d
\end{array}\right]=\left[\begin{array}{llll}
1 & x_{1} & y_{1} & z_{1} \\
1 & x_{2} & y_{2} & z_{2} \\
1 & x_{3} & y_{3} & z_{3} \\
1 & x_{3} & y_{4} & z_{4}
\end{array}\right]^{-1}\left[\begin{array}{l}
U_{1} \\
U_{2} \\
U_{3} \\
U_{4}
\end{array}\right]
$$

Let the inverse of the coefficient matrix be denoted by $D$, 


$$
\mathrm{D}=\left[\begin{array}{llll}
1 & x_{1} & y_{1} & z_{1} \\
1 & x_{2} & y_{2} & z_{2} \\
1 & x_{3} & y_{3} & z_{3} \\
1 & x_{3} & y_{4} & z_{4}
\end{array}\right]^{-1}=\left[\begin{array}{llll}
D_{1} & D_{2} & D_{3} & D_{4}
\end{array}\right] / \operatorname{det}(\mathrm{D})
$$

With $D_{i}$ being the following column vectors,

$$
\begin{aligned}
& D_{1}=\left[\begin{array}{c}
-\left(x_{2} y_{3} z_{4}-x_{2} z_{3} y_{4}-x_{3} y_{2} z_{4}+x_{3} z_{2} y_{4}+x_{4} y_{2} z_{3}-x_{4} z_{2} y_{3}\right) \\
\left(y_{3} z_{4}-z_{3} y_{4}-y_{2} z_{4}+z_{2} y_{4}+y_{2} z_{3}-z_{2} y_{3}\right) \\
-\left(x_{3} z_{4}-z_{3} x_{4}-x_{2} z_{4}+z_{2} x_{4}+x_{2} z_{3}-z_{2} x_{3}\right) \\
\left(x_{3} y_{4}-y_{3} x_{4}-x_{2} y_{4}+y_{2} x_{4}+x_{2} y_{3}-y_{2} x_{3}\right)
\end{array}\right] \\
& D_{2}=\left[\begin{array}{c}
\left(x_{1} y_{3} z_{4}-x_{1} z_{3} y_{4}-x_{3} y_{1} z_{4}+x_{3} z_{1} y_{4}+x_{4} y_{1} z_{3}-x_{4} z_{1} y_{3}\right) \\
-\left(y_{3} z_{4}-z_{3} y_{4}-y_{1} z_{4}+z_{1} y_{4}+y_{1} z_{3}-z_{1} y_{3}\right) \\
\left(x_{3} z_{4}-z_{3} x_{4}-x_{1} z_{4}+z_{1} x_{4}+x_{1} z_{3}-z_{1} x_{3}\right) \\
-\left(x_{3} y_{4}-y_{3} x_{4}-x_{1} y_{4}+y_{1} x_{4}+x_{1} y_{3}-y_{1} x_{3}\right)
\end{array}\right] \\
& D_{3}=\left[\begin{array}{c}
-\left(x_{1} y_{2} z_{4}-x_{1} z_{2} y_{4}-x_{2} y_{1} z_{4}+x_{2} z_{1} y_{4}+x_{4} y_{1} z_{4}-x_{4} z_{1} y_{2}\right) \\
\left(y_{2} z_{4}-z_{2} y_{4}-y_{1} z_{4}+z_{1} y_{4}+y_{1} z_{2}-z_{1} y_{2}\right) \\
-\left(x_{2} z_{4}-z_{2} x_{4}-x_{1} z_{4}+z_{1} x_{4}+x_{1} z_{2}-z_{1} x_{2}\right) \\
\left(x_{2} y_{4}-2 x_{4}-x_{1} y_{4}+y_{1} x_{4}+x_{1} y_{2}-y_{1} x_{2}\right)
\end{array}\right] \\
& D_{4}=\left[\begin{array}{c}
\left(x_{1} y_{2} z_{3}-x_{1} z_{2} y_{3}-x_{2} y_{1} z_{3}+x_{2} z_{1} y_{3}+x_{3} y_{1} z_{2}-x_{3} z_{1} y_{2}\right) \\
-\left(y_{2} z_{3}-z_{2} y_{3}-y_{1} z_{3}+z_{1} y_{3}+y_{1} z_{2}-z_{1} y_{2}\right) \\
\left(x_{2} z_{3}-z_{2} x_{3}-x_{1} z_{3}+z_{1} x_{3}+x_{1} z_{2}-z_{1} x_{2}\right) \\
-\left(x_{2} y_{3}-y_{2} x_{3}-x_{1} y_{3}+y_{1} x_{3}+x_{1} y_{2}-y_{1} x_{2}\right)
\end{array}\right]
\end{aligned}
$$

The determinant of $D$ is equal to six times the volume of the tetrahedron $V$.

$$
\begin{array}{r}
\operatorname{det}(\mathbf{D})=6 V=x_{2} y_{3} z_{4}-x_{2} z_{3} y_{4}-x_{3} y_{2} z_{4}+x_{3} z_{2} y_{4}+x_{4} y_{2} z_{3}-x_{4} z_{2} y_{3}+x_{1} y_{3} z_{4}-x_{1} z_{3} y_{4} \\
\ldots-x_{3} y_{1} z_{4}+x_{3} z_{1} y_{4}+x_{4} y_{1} z_{3}-x_{4} z_{1} y_{3}+x_{1} y_{2} z_{4}-x_{1} z_{2} y_{4}-x_{2} y_{1} z_{4}+x_{2} z_{1} y_{4} \\
\ldots+x_{4} y_{1} z_{4}-x_{4} z_{1} y_{2}+x_{1} y_{2} z_{3}-x_{1} z_{2} y_{3}-x_{2} y_{1} z_{3}+x_{2} z_{1} y_{3}+x_{3} y_{1} z_{2}-x_{3} z_{1} y_{2}
\end{array}
$$

Substituting the above terms into (5-17) gives the potential over the element,

$$
U(x, y, z)=\left[\begin{array}{llll}
1 & x & y & z
\end{array}\right]\left[\begin{array}{llll}
D_{11} & D_{12} & D_{13} & D_{14} \\
D_{21} & D_{22} & D_{23} & D_{24} \\
D_{31} & D_{32} & D_{33} & D_{34} \\
D_{41} & D_{42} & D_{43} & D_{44}
\end{array}\right]\left[\begin{array}{l}
U_{1} \\
U_{2} \\
U_{3} \\
U_{4}
\end{array}\right]
$$

where $D_{i j}$ are the elements of $\mathbf{D}$. This equation can be written as

$$
U(x, y, z)=\sum_{i=1}^{4} U_{i} \phi_{i}(x, y, x)
$$


In (5-21), interpolation functions, $\phi_{i}(x, y, x) i \in(1,2,3,4)$ are defined as,

$$
\phi_{i}(x, y, x)=D_{1 i}+D_{2 i} x+D_{3 i} y+D_{4 i} z=\frac{1}{6 V}\left(D_{1 i}+D_{2 i} x+D_{3 i} y+D_{4 i} z\right)
$$

This interpolatory function $\phi_{i}$ have the property:

$$
\phi_{i}\left(x_{j}, y_{j}\right)= \begin{cases}1 & i=j \\ 0 & i \neq j\end{cases}
$$

\subsection{Methods of Weighted Residuals}

The potential within the mesh $\tilde{u}$ is a linear combination of a piecewise linear interpolation $\phi_{i}$ function weighted by the potential at the nodes $u_{i}$.

$$
\tilde{u}(\vec{x})=\sum_{i=1}^{N} u_{i} \phi_{i}(\vec{x}) \quad \text { where } \phi_{i}=\left\{\begin{array}{l}
1 \text { on vertex } i \\
0 \text { elsewhere }
\end{array}\right.
$$

Equation 5-23 is a finite approximate of the potential. The Laplace equation would not be zero due to the error or 'residual' produced from using the interpolation function. Therefore, the method of weighted residual is used to obtain solution of the FEM by deriving weak form of the Laplace equation through multiplication of the equation 5-8 with some weighted function ' $v$ ' and then integrating over the domain $\Omega$ as expressed in equation $5-24$.

$$
\int_{\Omega} v(\nabla(\sigma \nabla \tilde{u})) d \Omega=0
$$

Here $v$ is an arbitrary function that weighs the residual such that it is zero in some weighted or average sense. Among several methods used in weighting residuals, the Galerkin method of weighted residuals is used here since the weighted function $v$ uses the same interpolation function $\phi_{i}$ as in equation 5-21, i.e.

$$
v(\vec{x})=\sum_{i=1}^{N} w_{i} \phi_{i}(\vec{x})
$$

where $w_{i}$ are the coefficients that weigh the interpolation functions $\phi_{i}$. 
By substituting the value of $v$ in (5-24) and applying the vector derivative identity, the second-order derivative can be separated into two terms as,

$$
[\nabla \cdot(v \sigma \nabla \widetilde{u})]=\sigma \nabla \tilde{u} \cdot \nabla v+v \nabla \cdot(\sigma \nabla \widetilde{u})
$$

Substitution of equation (5-26) in (5-24) and rearranging gives,

$$
\int_{\Omega} \nabla \cdot(v \sigma \nabla \tilde{u}) d \Omega=\int_{\Omega} \sigma \nabla \tilde{u} \cdot \nabla v d \Omega
$$

Applying Divergence theorem to the left hand side of the equation permits the introduction of boundary conditions,

$$
\int_{\Omega} \nabla \cdot F d V=\int_{\partial V} F d S \rightarrow \int_{\partial \Omega} v \sigma \nabla \tilde{u} \cdot \widehat{n} d \Gamma=\int_{\Omega} \sigma \nabla \tilde{u} \cdot \nabla v d \Omega
$$

Since $\nabla \phi \cdot \hat{n}=\frac{\partial \phi}{\partial \hat{n}}$, the above equation can be written as

$$
\int_{\partial \Omega} v \sigma \frac{\partial \tilde{u}}{\partial \tilde{\tau}} d \Gamma=\int_{\Omega} \sigma \nabla \tilde{u} \cdot \nabla v d \Omega
$$

The right hand side of the equation is applied for the entire mesh whereas the boundary integral is carried out only for the elements underneath the electrodes.

Substituting the values of $\tilde{u}$ and $v$ in equation (5-29) and examining for a single tetrahedral element $k$, the right hand term can be represented as,

$$
\int_{E_{k}} \sigma_{k} \nabla \tilde{u} \cdot \nabla v d \Omega=\int_{E_{k}} \sigma_{k} \nabla \sum_{i=1}^{4} u_{i} \phi_{i}(\vec{x}) \cdot \nabla \sum_{j=1}^{4} w_{j} \tilde{u}_{j}(\vec{x}) d \Omega
$$

Since the conductivity $\sigma_{k}$ is constant over an element, nodal voltages $u_{i r}$ the weighing function coefficients and the summations can be moved outside the integral,

$$
\sigma_{k} \sum_{i=1}^{4} u_{i} \sum_{j=1}^{4} w_{j} \int_{E_{k}} \nabla \phi_{i} \cdot \nabla \phi_{j} d \Omega
$$

Applying the equation over the entire domain $\Omega$,

$$
\int_{\Omega} \sigma \nabla \tilde{u} \cdot \nabla v d \Omega=\sum_{E=1}^{K} \sigma_{k} \sum_{i=1}^{4} \sum_{j=1}^{4} u_{j} S_{i j}^{k}
$$


where $S_{i j}^{k}$ the local matrix of the element defined as $\int_{E_{k}} \nabla \phi_{i} \cdot \nabla \phi_{j} d \Omega$

Likewise, the boundary integral term can be written as,

$$
\int_{\partial \Omega} v \sigma \frac{\partial \tilde{u}}{\partial \tilde{n}} d \Gamma=\sigma_{k} \sum_{i=1}^{4} \sum_{j=1}^{4} w_{j} \int_{\partial \Omega} \phi_{i} \nabla \phi_{j} \cdot \widehat{n} d \Gamma
$$

\subsection{Implementation of CEM}

Equation 5-14, 5-15, 5-16, 5-32 and 5-33 can be combined to form a system of equations for CEM which can be written as,

$$
\left[\begin{array}{cc}
A_{c 1}+A_{c 2} & A_{e} \\
A_{e}^{T} & A_{d}
\end{array}\right]\left[\begin{array}{l}
u \\
U
\end{array}\right]=\left[\begin{array}{l}
0 \\
1
\end{array}\right]
$$

Here, $u$ is the vector of unknown nodal potentials, $\mathbf{U}$ is the vector of unknown potentials on the electrodes. $A_{c 1}$ is the system matrix (identical to $Y$ defined in equation 5.12),

$$
A_{c 1}=\int_{\Omega} \nabla \phi_{i} \cdot \nabla \phi_{j} d \Omega
$$

Since $\boldsymbol{A}_{\mathbf{c 1}}$ does not include any information on the boundary condition, $\boldsymbol{A}_{\mathbf{c} 2}$ is added to represent the effect of the contact impedance on the system matrix.

$$
A_{c 2}(i, j)=\sum_{l=1}^{L} \frac{1}{z_{j}} \int_{e_{l}} \phi_{i} \phi_{j} d \Gamma
$$

and $\boldsymbol{A}_{\boldsymbol{e}}$ represents the weights of the voltage on the electrodes,

$$
A_{e}(i, j)=-\frac{1}{z_{j}} \int_{e_{l}} \phi_{i} d \Gamma i \in[1, N] \text { and } j \in[1, L]
$$

Finally $\boldsymbol{A}_{\boldsymbol{d}}$ is a diagonal matrix where the values of the main diagonal are the ratios of the electrode lengths $\left|e_{l}\right|$ to respective contact impedance,

$$
A_{d}(i, j)=\left\{\begin{array}{cl}
\left|e_{l}\right| \frac{1}{z_{l}} & \text { for } i, j \in[1, L] \\
0 & \text { otherwise }
\end{array}\right.
$$


This mathematical formulation is implemented using EIDORS algorithm [56] in MATLAB where the system matrix $A=\left[\begin{array}{cc}A_{c 1}+A_{c 2} & A_{e} \\ A_{e}^{T} & A_{d}\end{array}\right]$ is calculated and Cholesky factorization is applied to solve for the nodal potentials $\mathbf{V}=\left[\begin{array}{l}u \\ u\end{array}\right]$. The nodal potentials are then used to determine the potential and current density in the elements representing the heart.

\subsection{Example of FEM}

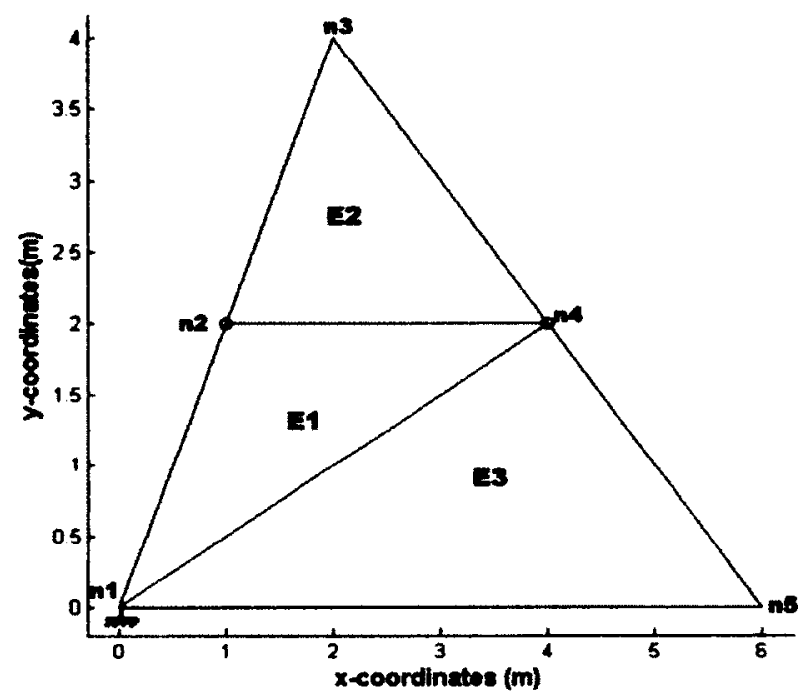

Figure 5.3 2-D FEM with 3 elements. Green circles represent point electrodes

The above 2-D mesh is constructed to illustrate the calculation of voltage and current density. The mesh has 5 nodes $n 1, n 2, n 3, n 4$, and $n 5$ with 3 elements $E 1, E 2$, and E3. Nodes representing $E 1$ are $n 1, n 2$ and $n 4$, for $E 2$ are $n 2, n 3$ and $n 4$, and for $E 3$ are $n 1, n 4$ and $n 5$. Conductivity for E1, E2 and E3 are assigned as $1 \mathrm{~S} / \mathrm{m}, 2 \mathrm{~S} / \mathrm{m}$ and $3 \mathrm{~S} / \mathrm{m}$ respectively. Node $n 1$ is grounded, and $n 2$ and $n 4$ represents point electrodes. Current of 3 $\mathrm{mA}$ was simulated in the electrode and using equations 5-11 to 5-13, voltages on each of the nodes were calculated as shown below.

The location of nodes are defined as,

$$
n 1=\left[\begin{array}{ll}
0 & 0
\end{array}\right], \quad n 2=\left[\begin{array}{ll}
1 & 2
\end{array}\right], \quad n 3=\left[\begin{array}{ll}
2 & 4
\end{array}\right], \quad n 4=\left[\begin{array}{ll}
4 & 2
\end{array}\right], \quad n 5=\left[\begin{array}{ll}
6 & 0
\end{array}\right]
$$

The location of the elements is, 


$$
e 1=\left[\begin{array}{l}
n_{1} \\
n_{4} \\
n_{2}
\end{array}\right], \quad e 2=\left[\begin{array}{l}
n_{2} \\
n_{4} \\
n_{3}
\end{array}\right], \quad e 3=\left[\begin{array}{l}
n_{1} \\
n_{5} \\
n_{4}
\end{array}\right]
$$

The connectivity matrix $C$ is,

$$
C=\left[\begin{array}{lllll}
1 & 0 & 0 & 0 & 0 \\
0 & 0 & 0 & 1 & 0 \\
0 & 1 & 0 & 0 & 0 \\
0 & 1 & 0 & 0 & 0 \\
0 & 0 & 0 & 1 & 0 \\
0 & 0 & 1 & 0 & 0 \\
1 & 0 & 0 & 0 & 0 \\
0 & 0 & 0 & 0 & 1 \\
0 & 0 & 0 & 1 & 0
\end{array}\right]
$$

The current vector is,

$$
Q=\left[\begin{array}{c}
0 \\
0.003 \\
0 \\
-0.003 \\
0
\end{array}\right] \text { Amperes }
$$

The inverse of matrix $A$ is determined which is defined as,

$$
\begin{aligned}
& A_{1}=\left[\begin{array}{ll}
1 & n 1 \\
1 & n 4 \\
1 & n 2
\end{array}\right]^{-1} \\
& A_{2}=\left[\begin{array}{ll}
1 & n 2 \\
1 & n 4 \\
1 & n 3
\end{array}\right]^{-1} \\
& A_{3}=\left[\begin{array}{ll}
1 & n 1 \\
1 & n 5 \\
1 & n 4
\end{array}\right]^{-1}
\end{aligned}
$$

The matrix $B_{1}, B_{2}$, and $B_{3}$ are created which is the submatrix of $A_{1}, A_{2}$, and $A_{3}$ with the top row deleted.

We now substitute these values in equation 5-11 which is,

$$
\begin{gathered}
S_{1}=\frac{B_{1}{ }^{T} B_{1}}{2\left|\operatorname{det} A_{1}\right|} \\
S_{2}=\frac{2 B_{2}{ }^{T} B_{2}}{2\left|\operatorname{det} A_{2}\right|}
\end{gathered}
$$




$$
S_{3}=\frac{3 B_{3}{ }^{r} B_{3}}{2\left|\operatorname{det} A_{3}\right|}
$$

The system matrix $Y$ is then calculated using 5-12.

$$
Y=C^{T} S C
$$

Here, $S$ is the block diagonal matrix of the matrices $S_{1}, S_{2}$ and $S_{3}$.

The voltages in the nodes are then calculated using 5-13

$$
V=Y^{-1} Q
$$

The voltage on $n 1, n 2, n 3, n 4$, and $n 5$ were reported to be $0 \mathrm{mV}, 0.6842 \mathrm{mV}, 0.1053 \mathrm{mV}$, $-1.0526 \mathrm{mV}$, and $-1.2632 \mathrm{mV}$ respectively.

Using these values and equations 5-17 and 5-5, the current density in the first element was calculated as,

$$
V_{e 1}(x, y)=\left[\begin{array}{lll}
1 & x_{4} & y_{4} \\
1 & x_{2} & y_{2}
\end{array}\right]^{-1}\left[\begin{array}{l}
U_{4} \\
U_{2}
\end{array}\right]
$$

where $V_{e 1}(x, y)$ is the potential gradient of $e 1, x_{2}, y_{2}$ are $x$ and $y$ co-ordinates of node $n 2$, $x_{4}, y_{4}$ are co-ordinates of $n 4$, and $U 2$ and $U 4$ voltages of the nodes $n 2$ and $n 4$ respectively.

$$
\begin{gathered}
J_{e 1}=\sigma_{e 1} * V_{e 1}(x, y) \\
=1 *\left[\begin{array}{c}
-0.5789 \\
0.6319
\end{array}\right] \\
=\left[\begin{array}{c}
-0.5789 \\
0.6316
\end{array}\right] \mathrm{mA} / \mathrm{m}^{2}
\end{gathered}
$$

The magnitude of the current density in then calculated as,

$$
\begin{gathered}
J=\sqrt{J_{e 1 x}^{2}+J_{e 1 y}^{2}} \\
=\sqrt{(-0.5789)^{2}+(0.6316)^{2}} \\
=0.857 \mathrm{~mA} / \mathrm{m}^{2}
\end{gathered}
$$

Similarly, the current density in element 2 and element 3 was calculated to be 1.1579 $\mathrm{mA} / \mathrm{m}^{2}$ and $0.7061 \mathrm{~mA} / \mathrm{m}^{2}$. 
In the same way, the current density in the heart for our 3-D thorax model is calculated.

\subsection{FEM Validation}

FEM is used in this work to simulate electrical activity in a human thorax model. To verify this approach, we compare the potential difference obtained from the simulation to the analytical solution.

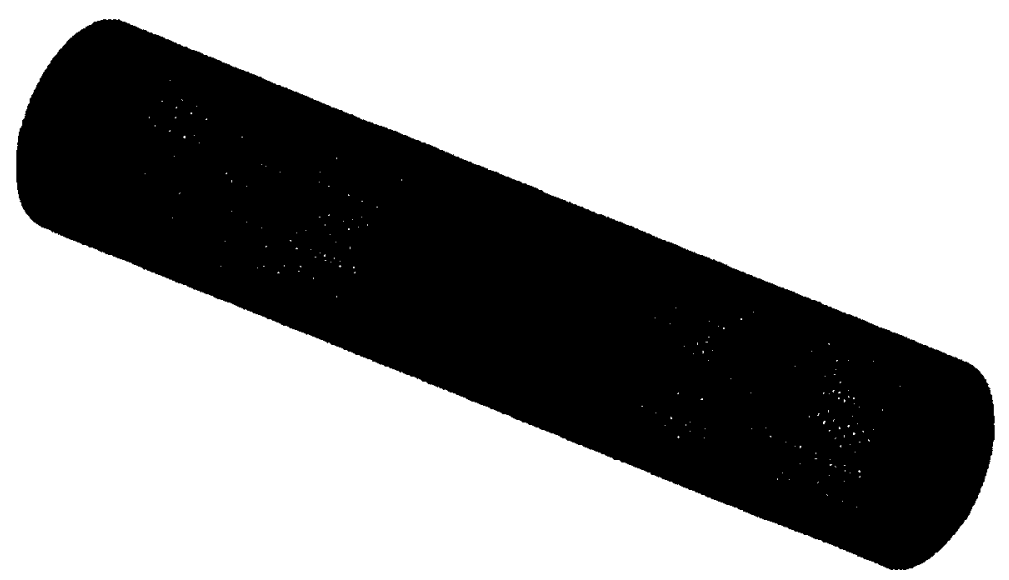

Figure 5.4 3-D FEM cylindrical model with two electrodes (in green) enclosing a smaller cylindrical object

Figure 5.4 represents a simple 3-D cylindrical model enclosing a smaller cylinder. Two circular electrodes are attached to the bases of the cylinder. The conductivity of the outer and inner cylinder is taken to be $1 \mathrm{~S} / \mathrm{m}$ and $5 \mathrm{~S} / \mathrm{m}$ respectively. Current amplitude of $2 \mathrm{~mA}$ is simulated and the potential difference was recorded.

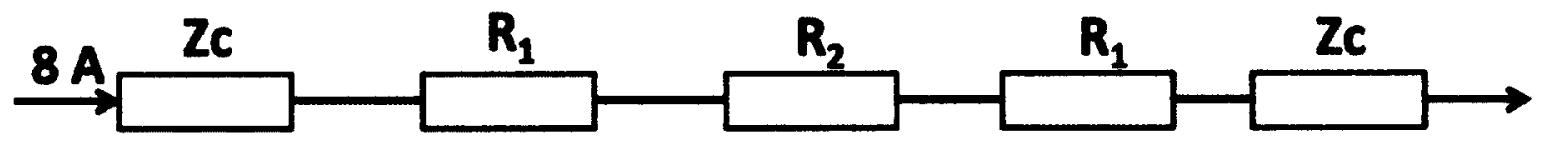

Figure 5.5 Electrical representation of the model

This 3-D model can be electrically represented as Figure 5.5 where $Z_{c}$ is the contact impedance, $R_{1}$ is the resistance offered by the outer cylinder and $R_{2}$ is the resistance of the inner cylinder.

The resistance is calculated by the formula,

$$
\boldsymbol{R}=\frac{l}{\sigma A}
$$


where $l$ is the length, $\sigma$ is the conductivity and $A$ is the area of the object.

For the analytical solution, the resistance is calculated and multiplied with the current to give the potential difference, as shown below

$$
V=I *\left(2 Z_{c}+2 R_{1}+R_{2}\right)
$$

The potential values from the analytical and FEM approach were compared and a relative error of $0.24 \%$ was found. This shows that the results are in good agreement and hence the FEM is validated for this type of problem. 


\section{Finite Element Method Results}

In this work, FEM was implemented to further the understanding of the electrical activity in the body during cardioversion for different individuals exhibiting different transthoracic impedances and also examining different positions of the electrodes that could be helpful in increasing the success of cardioversion.

This chapter describes the generation of the FEM mesh, the different defibrillation parameters calculated, the results on sizes and position, and their respective analyses.

\subsection{FEM Mesh}

\subsubsection{Mesh Generation}

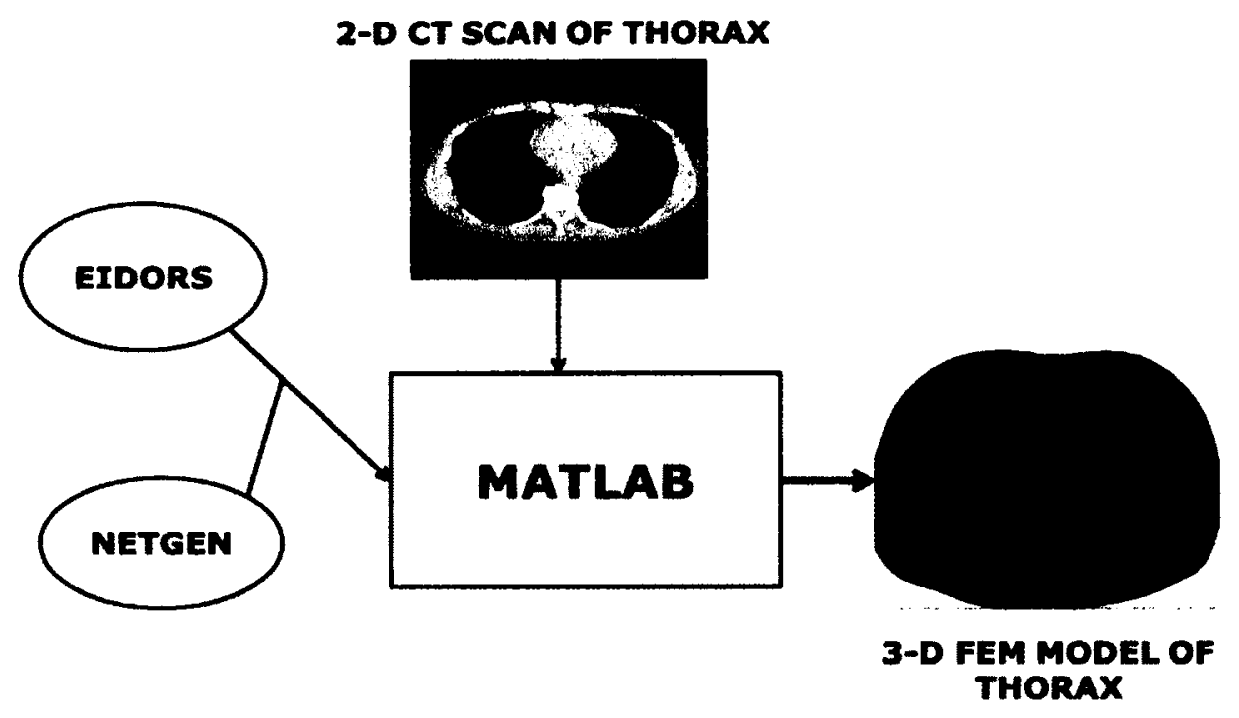

Figure 6.1 Summary of the FEM mesh generation process

The FEM models for this work are constructed using the Electrical Impedance and Diffuse Optics Reconstruction Software (EIDORS) package [80] running within the MATLAB R2012b environment. EIDORS $\mathrm{v} 3.5$ is interfaced to FEM generation tool Netgen [79] which 
constructs the model using the contours extracted from the 2-D thoracic cross-sectional Computerized Axial Tomographic (CAT) scan data. Assuming that the body is translationally symmetric in z-direction (vertical direction), 3-D model of the chest comprising of the thorax wall and lungs is constructed from the 2-D image. The translational symmetry assumption holds only for lungs, thorax and fat tissue in the model.

The FEM algorithms used were already available in EIDORS. Those existing algorithms were modified to incorporate internal anatomical structures mentioned above. The model was also modified to simulate different pad positions used during cardioversion. In addition, the algorithm was customized to include different conductivities of the tissues and calculate various defibrillation parameters mentioned in section 6.2 .

\subsubsection{Electrodes}

Two electrodes are modelled on the surface of the thorax wall to deliver the current in the heart. They are rectangular electrodes of size $11.1 \mathrm{~cm} \times 6.9 \mathrm{~cm}$, similar to the Kendall Medi-Trace 1710H Defibrillation Electrodes (Figure 6.2).

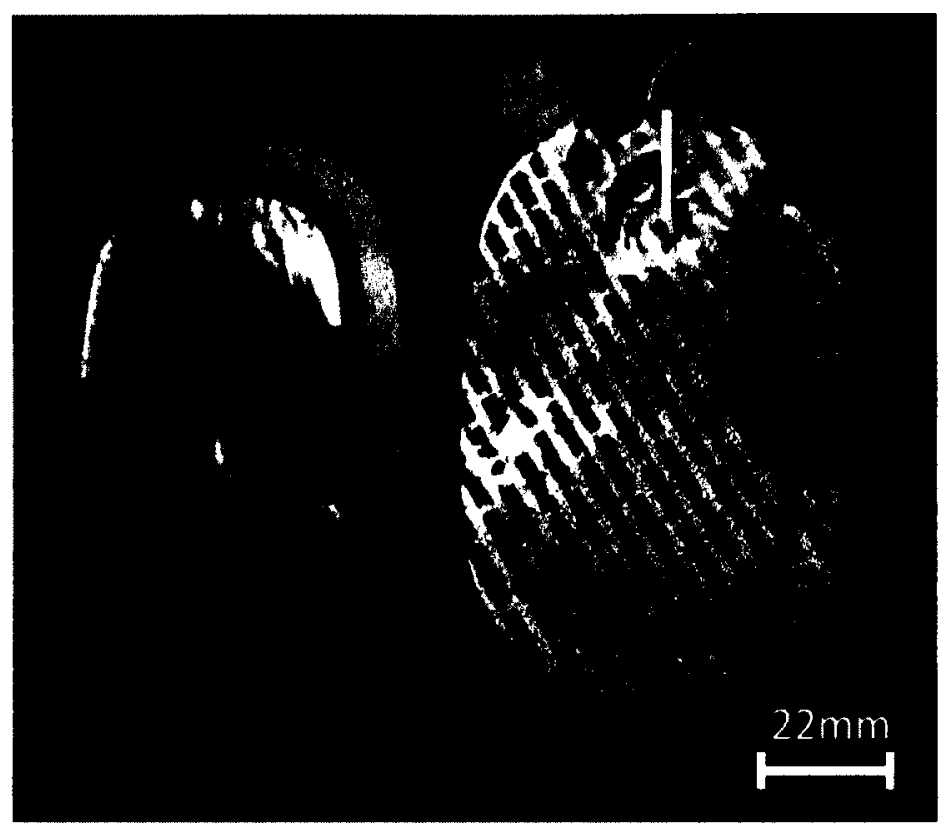

Figure 6.2 Kendall Medl-Trace 1710H Deflbrillation Electrodes used during defibrillation 


\subsubsection{Sensitivity}

The model has been constrained to be isotropic (i.e., the conductivity of the tissues are the same in all the directions). Therefore, a scalar conductivity value is assigned to each element that corresponds to the region in the body that the element represents. The model takes into account six (6) different types of tissue: cardiac muscle, lung, bone, fat, skin, and soft tissue.

Different literature reports different conductivity values for the lungs and the heart, as shown in Table 6.1.

Table 6.1 Conductivity values reported for lungs and heart

\begin{tabular}{|c|c|c|c|c|c|}
\hline Tissue & \multicolumn{5}{|c|}{ Conductivity (S/mm) } \\
\hline 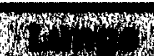 & 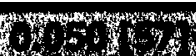 & 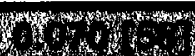 & & 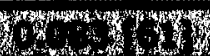 & 4 \\
\hline Heart & $0.167[57]$ & $0.220[82]$ & $0.250[58]$ & $0.500[61]$ & \\
\hline
\end{tabular}

The mean, maximum and minimum of the conductivity values of the heart and lungs were simulated in the FEM model to select the optimal conductivity for these tissues. The FEM results obtained for these conductivities are shown in table 6.2

Table 6.2 FEM results for Mean, maximum and minimum conductivity for lungs and heart

\begin{tabular}{|c|c|c|c|c|}
\hline Conductivity & $\begin{array}{c}\text { Mean } \\
\text { Current } \\
\text { Density } \\
\left(\mathbf{m A} / \mathbf{m m}^{2}\right)\end{array}$ & $\begin{array}{c}\text { Maximum } \\
\text { Current } \\
\text { Density } \\
\left(\mathbf{m A} / \mathbf{m m}^{2}\right)\end{array}$ & $\begin{array}{c}\text { Minimum } \\
\text { current } \\
\text { density } \\
\left(\mathbf{m A} / \mathbf{m m}^{2}\right)\end{array}$ & $\begin{array}{c}\text { Minimum } \\
\text { Current for } \\
\text { defibrillation } \\
\text { (A) }\end{array}$ \\
\hline Maximum & 0.5757 & 1.3860 & 0.4245 & 12.8 \\
\hline
\end{tabular}

From Table 6.2, we can see quite a large variation in these electrical parameters which indicates that these parameters are very sensitive to the conductivity values. Therefore, it is important to choose an optimal conductivity to obtain a good approximation of the defibrillation parameters. For our work, we selected the conductivity by simulating all the 
values reported in table 6.1. The potential difference for each of these conductivities were calculated and compared with the experimental voltage recorded from the HeartStart XL defibrillator (Refer to section 3.5 in Chapter 3). It can be seen from section 3.5, that voltages between $1350 \mathrm{~V}$ and $1950 \mathrm{~V}$ are required to drive a current of $25 \mathrm{~A}$. For conductivities of $0.089 \mathrm{~S} / \mathrm{mm}$ and $0.500 \mathrm{~S} / \mathrm{mm}$ for the lungs and heart (maximum conductivity values), respectively the voltage falls in the range of experimental values. The impedance of the electrodes was taken to be $50 \Omega$, as reported in the literature [83]. The conductivities of the tissues considered for this work are listed in Figure 6.3.

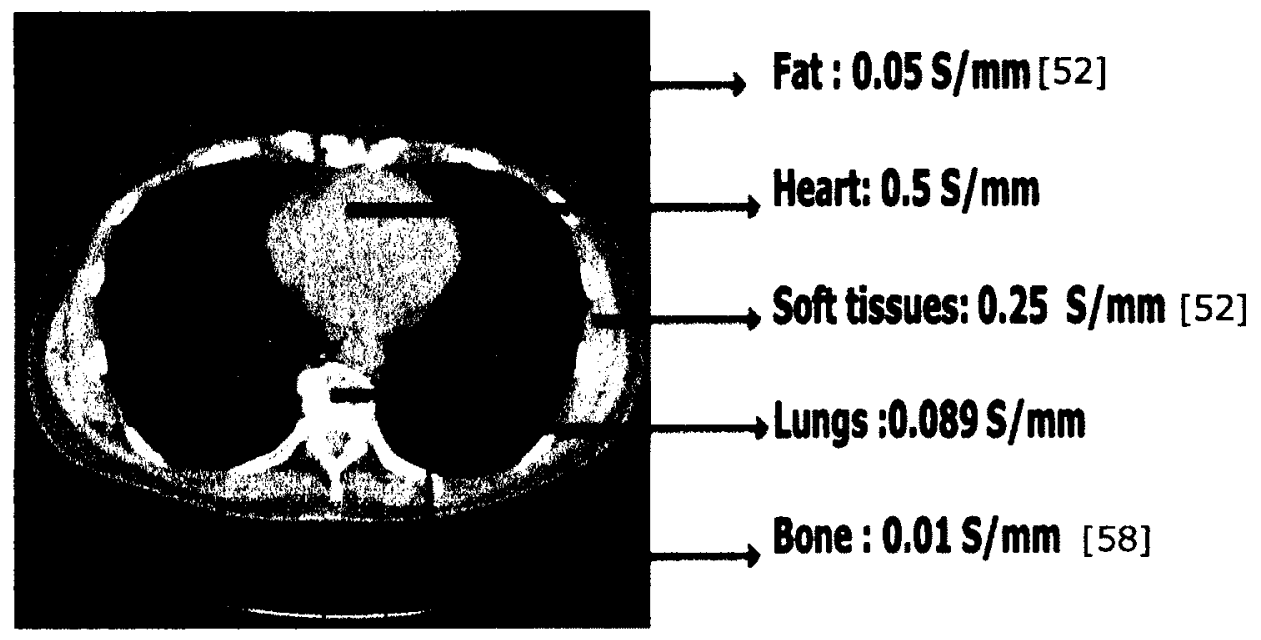

Figure 6.3 Conductivity of different tissue regions

\subsection{Computation of Defibrillation Parameters}

For our analysis on different patient types/sizes and pad positions, the following parameters are calculated.

1. Mean, maximum and minimum current density in the heart: These parameters are determined by passing a current of $25 \mathrm{~A}$ through electrodes. This input current is considered the average of the range of current required for a successful defibrillation for a biphasic defibrillator. The current density in each of the elements representing the heart is calculated and the average, maximum and minimum values are determined (Figure 6.4). 


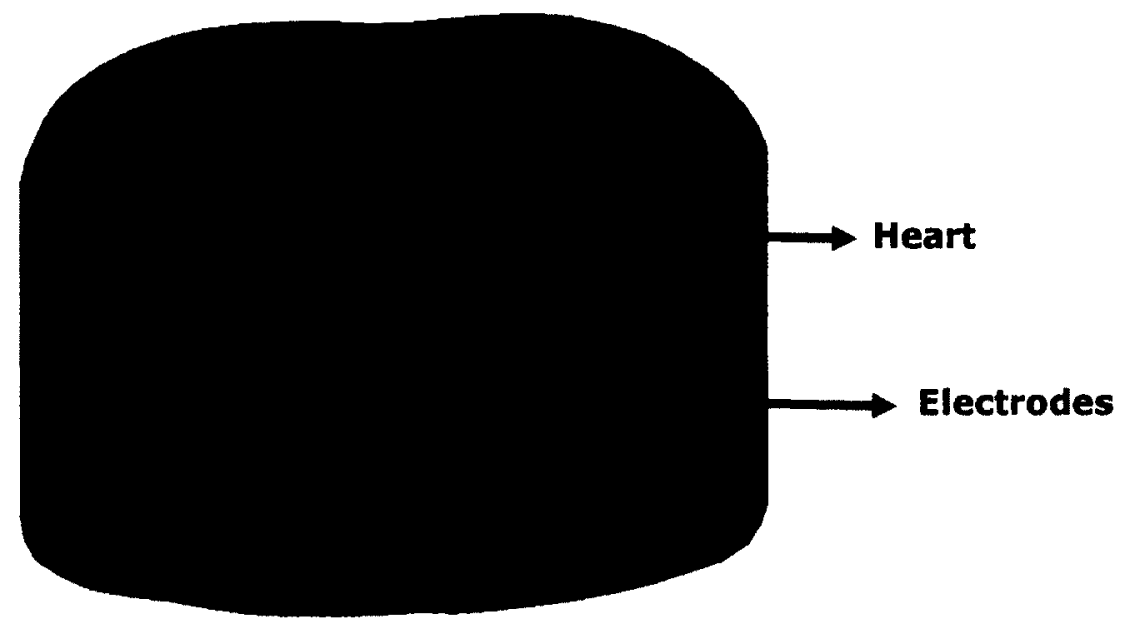

Figure 6.4 Thorax Model showing only the heart. Elements colored in maroon represents the heart region and current density is calculated in those blue elements.

2. Minimum voltage, current and energy required for a successful defibrillation: Studies have shown that defibrillation is successful only when an electric field of $5 \mathrm{~V} / \mathrm{cm}$ is achieved for $95 \%$ of the heart. From this information, the minimum current density required to defibrillate the heart $J_{t h}$ was calculated as shown below.

$$
\begin{gathered}
J=\sigma E \\
J_{\text {th }}=0.5 \frac{\mathrm{S}}{\mathrm{mm}} * 0.5 \frac{\mathrm{V}}{\mathrm{mm}}=0.25 \mathrm{~mA} / \mathrm{mm}^{2}
\end{gathered}
$$

The current where $95 \%$ of the elements representing the heart showed at least $0.25 \mathrm{~mA} / \mathrm{mm}^{2}$ of current density was taken to be the minimum current and simultaneously the voltage for that given current amplitude was taken as the minimum voltage. The minimum energy, or defibrillation threshold energy (DFT) is calculated using the energy equation, $E=\frac{1}{2} C V^{2}$ where $C$ is the capacitance, measured as $100 \mu \mathrm{F}$ for the HeartStart MRX defibrillator [84], and $V$ is the minimum potential difference across the electrodes that defibrillate $95 \%$ of the heart.

3. Uniformity of current density: Uniformity of the current density distribution in the myocardium is essential to avoid re-fibrillation. This is measured using the Heterogeneity Index ( $\mathrm{HI}) . \mathrm{HI}$ is a normalized estimate of heterogeneity and has been used as a measure of uniformity in various defibrillation studies [52][85][43] . 


$$
\mathbf{H I}=\frac{\mathbf{P}_{95-5}}{\mathbf{P}_{50}}
$$

where, $P_{5}, P_{50}$, and $P_{95}$ is the $5^{\text {th }}, 50^{\text {th }}$, and $95^{\text {th }}$ percentile respectively of current density (Figure 6.5).

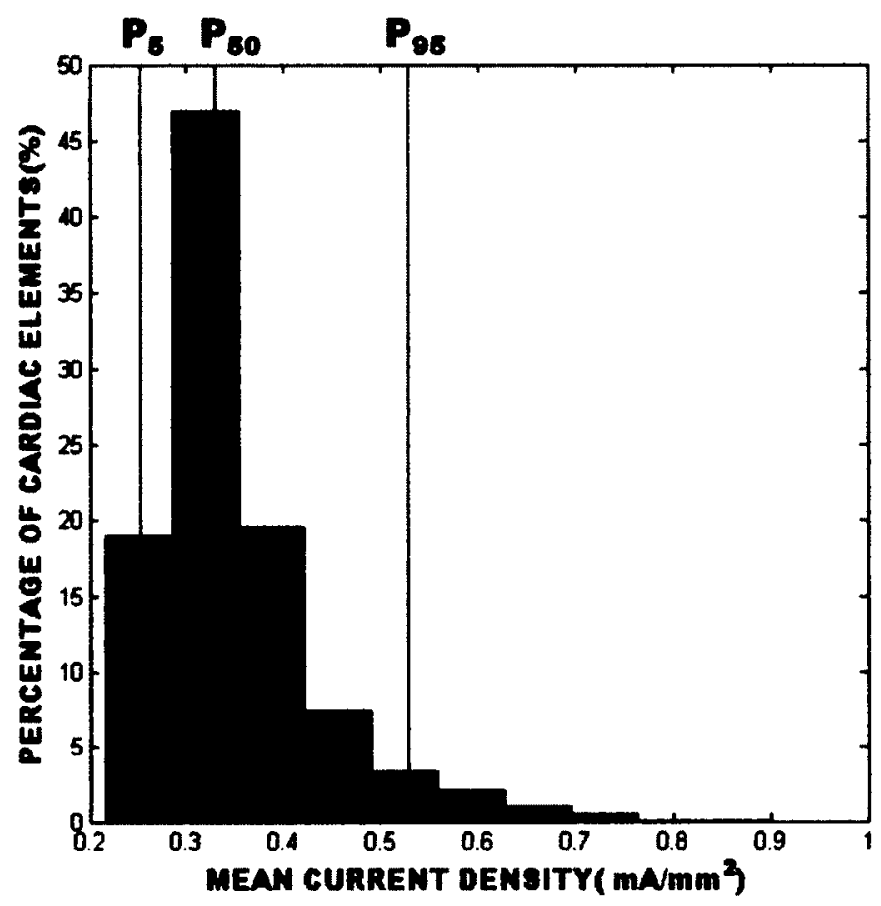

Figure 6.5 Calculation of Heterogeneity Index of the mean current density in the heart.

4. Percentage of cardiac damage: Potential gradients of approximately $50 \mathrm{~V} / \mathrm{cm}$ or higher have been shown to damage the cardiac cells [19][20]. Using equation 6-2, it was found that the current density in the heart of $2.5 \mathrm{~mA} / \mathrm{mm}^{2}$ or greater results in cardiac damage. The number of heart elements having current density greater than this value is divided by the total number of heart elements to calculate the percentage of cardiac damage in the heart. 


\subsection{Effect of different patient size and impedance on the current}

\section{distribution in the heart}

\subsubsection{Modelling}

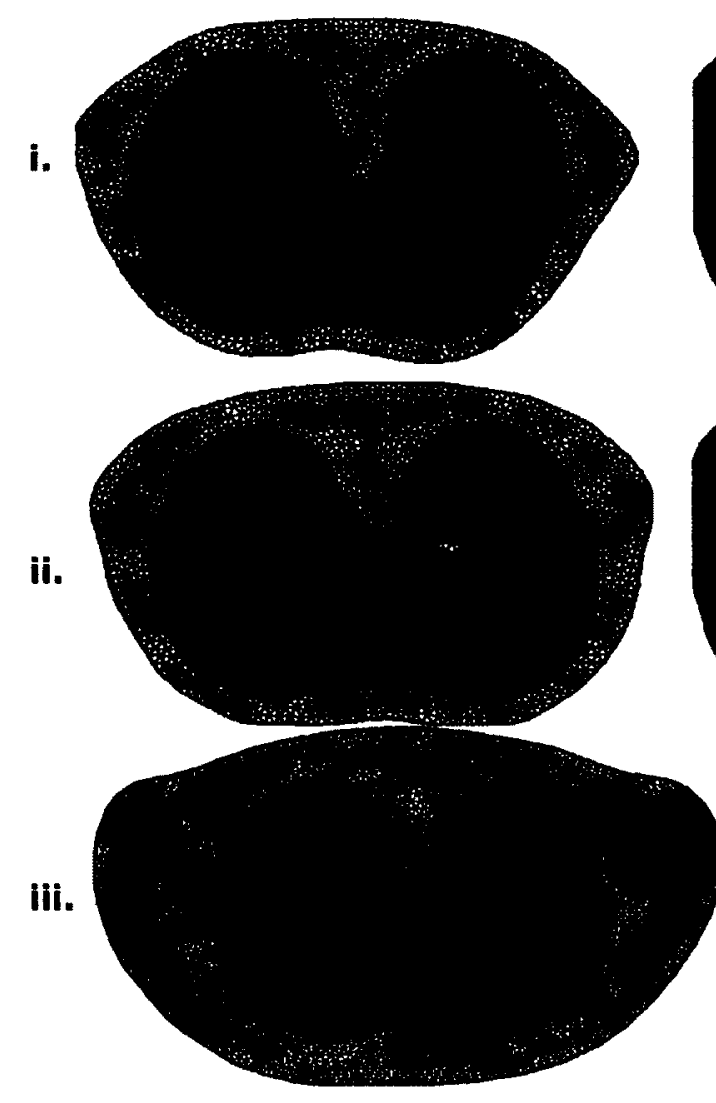

(a)
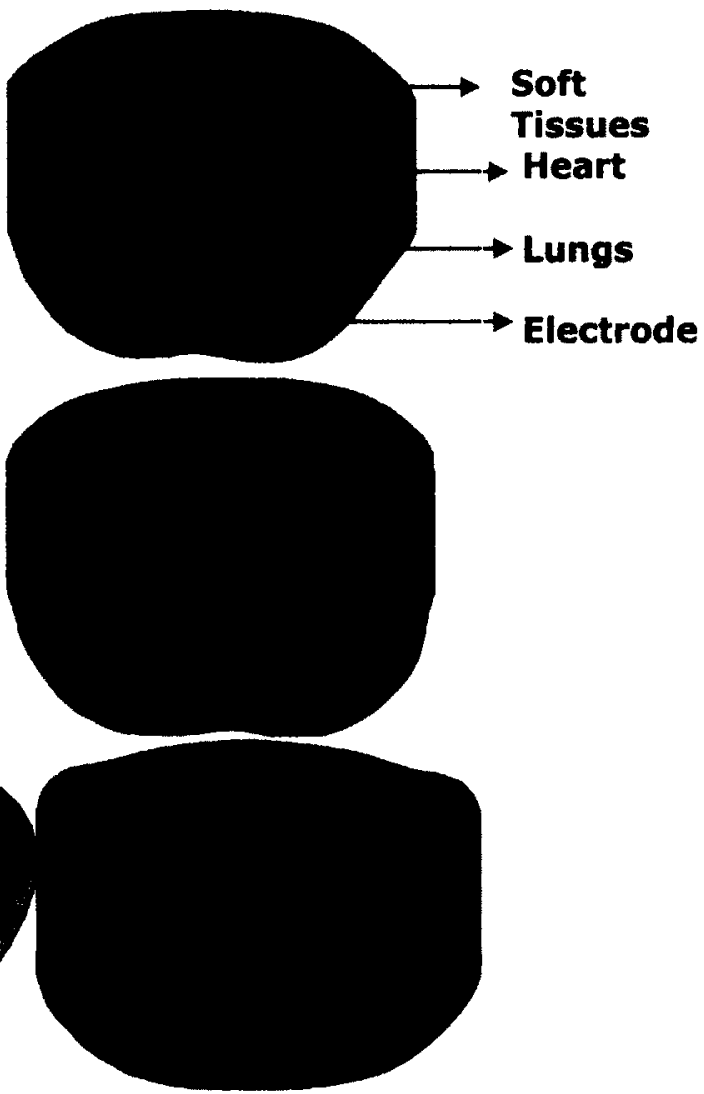

(b)

Figure 6.6 FEM Models of a i. thin, ii. normal, and ii. larger patients. a. Vlew from above $b$. View from front. Colours blue represent lungs, maroon - heart, green-electrodes, and white- soft tissues.

2-D CAT scans at T8 level were taken from three 17 years old male subjects weighing 50.3 $\mathrm{kg}, 67.5 \mathrm{~kg}$ and $125 \mathrm{~kg}$ (Credit: CT data were measured by Christian Woitzik at University of Giessen, Germany). We chose the CAT scans of same sex and age to avoid any bias in the results that may occur due to the difference in these demographics. The Body Mass Index figures of these patients were measured to be $17.51,21.3$ and $34.8 \mathrm{~kg} / \mathrm{m}^{2}$ classifying them in the thin, normal, and large (obese) category respectively. To preclude misinterpretation of the FEM results that may occur due to the difference in the height, the 
contours extracted from their scans were scaled to a height of 1.695 metres, which is the height of the thin patient. The electrodes were placed in AP2 position and FEM models were generated for the three cases, as shown in Figure 6.6. For this comparative analysis, the model was constituted of lungs, heart, soft tissue, and fatty tissue.

\subsubsection{Results}

The current density and voltage distributions for a thin, normal and large patient are shown in Figure 6.7. The mean current density for a current input of $25 \mathrm{~A}$ were reported to be $1.3315 \mathrm{~mA} / \mathrm{mm}^{2}$ for the thin patient, $1.1562 \mathrm{~mA} / \mathrm{mm}^{2}$ for the normal patient and $0.8089 \mathrm{~mA} / \mathrm{mm}^{2}$ for the large patient. No cardiac damage was seen for the large patient while $1.33 \%$ cardiac damage was seen for thin patient and $0.32 \%$ for the normal sized person.

The minimum current and energy required to achieve successful resuscitation is shown in Figure 6.9. It can be seen that larger patients require more current and energy than thin and normal patients. Between thin and normal sized patients, there does not seem to be a big difference in their current and energy requirement. 


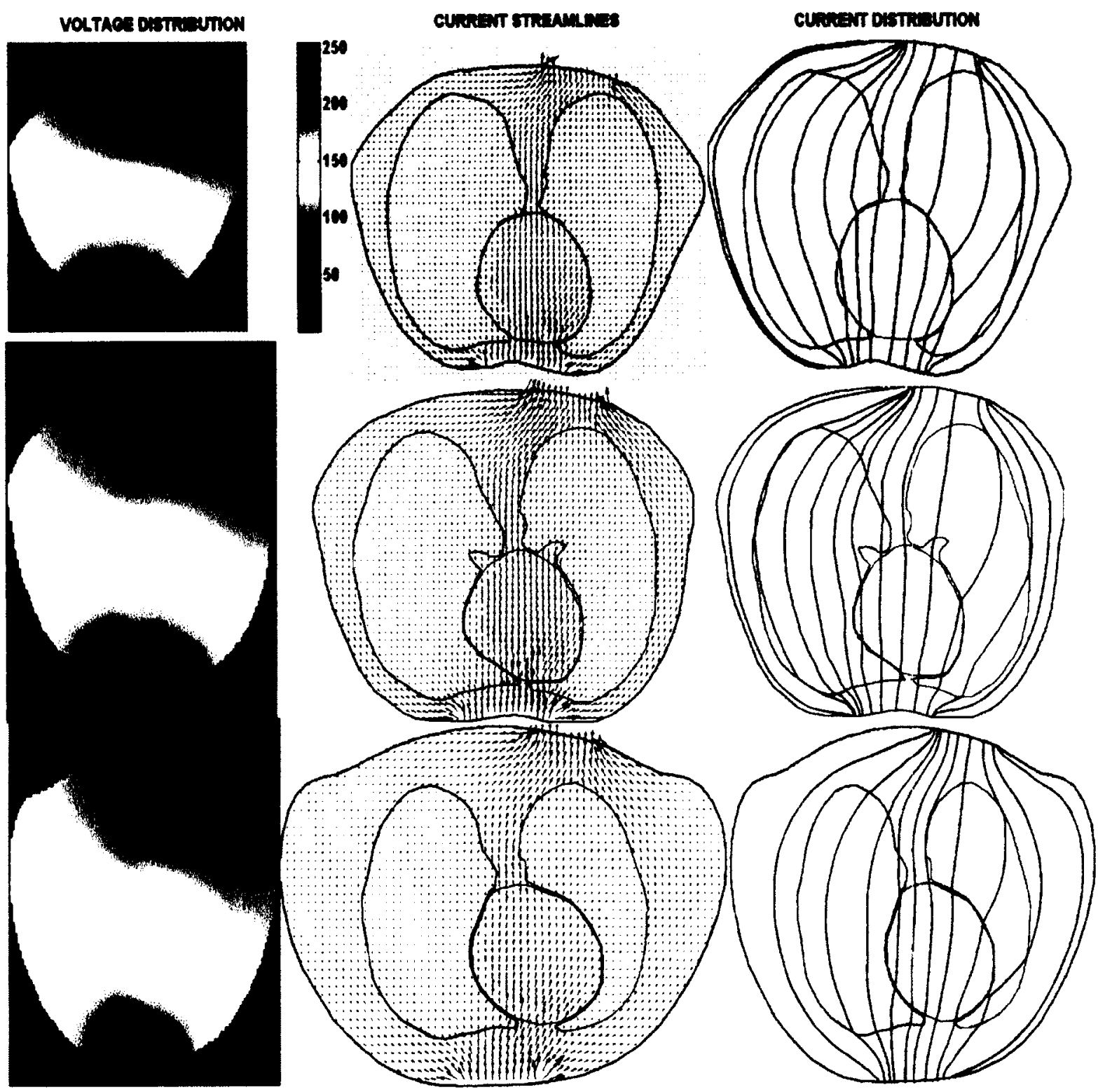

Figure 6.7 Voltage, and Current streamlines and distribution in a central slice for $a$. Thin, $b$. Normal, and $c$. Large sized person 


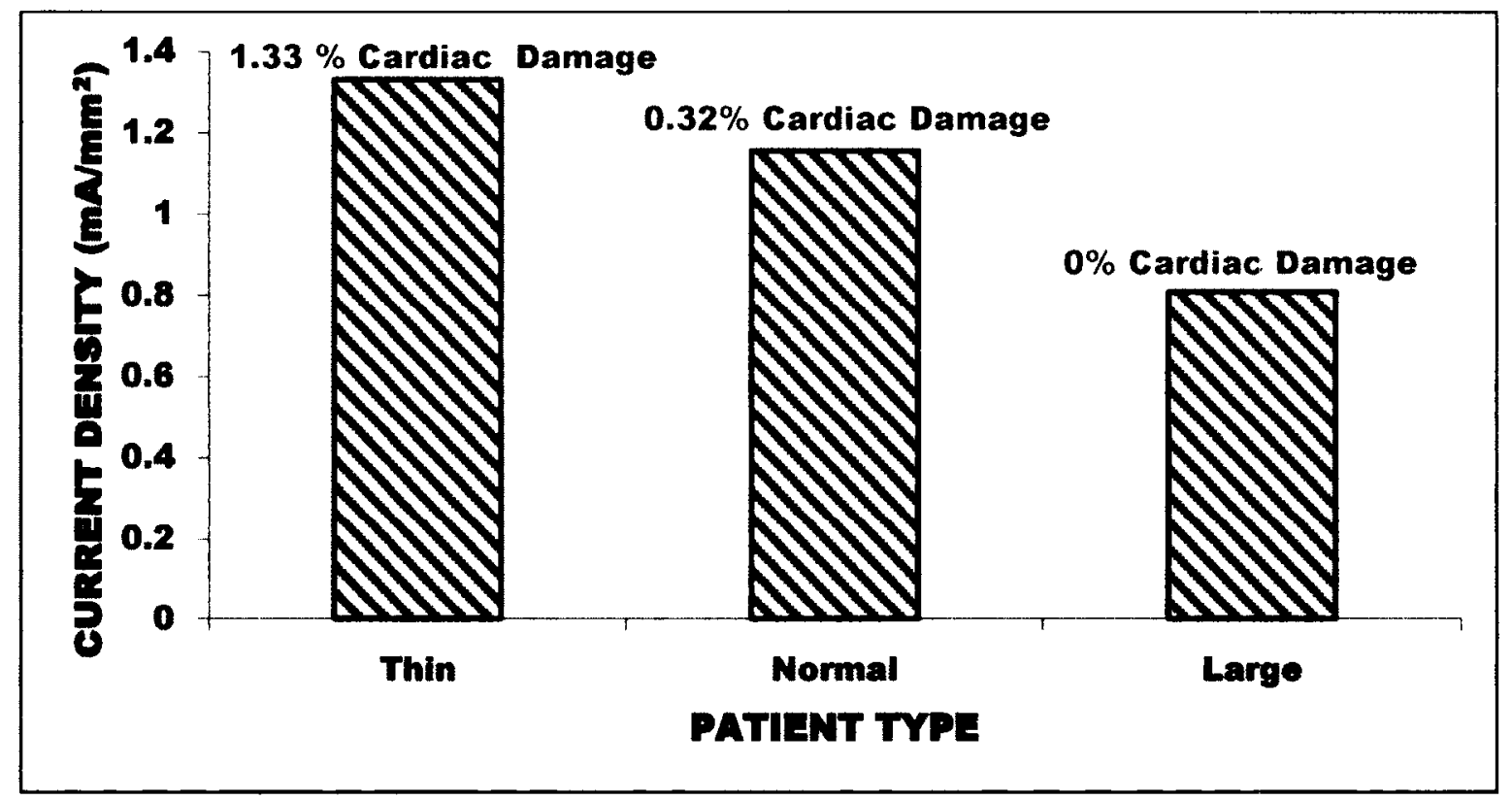

Figure 6.8 Mean Current Density for thin, normal, and large person for 25A input current.

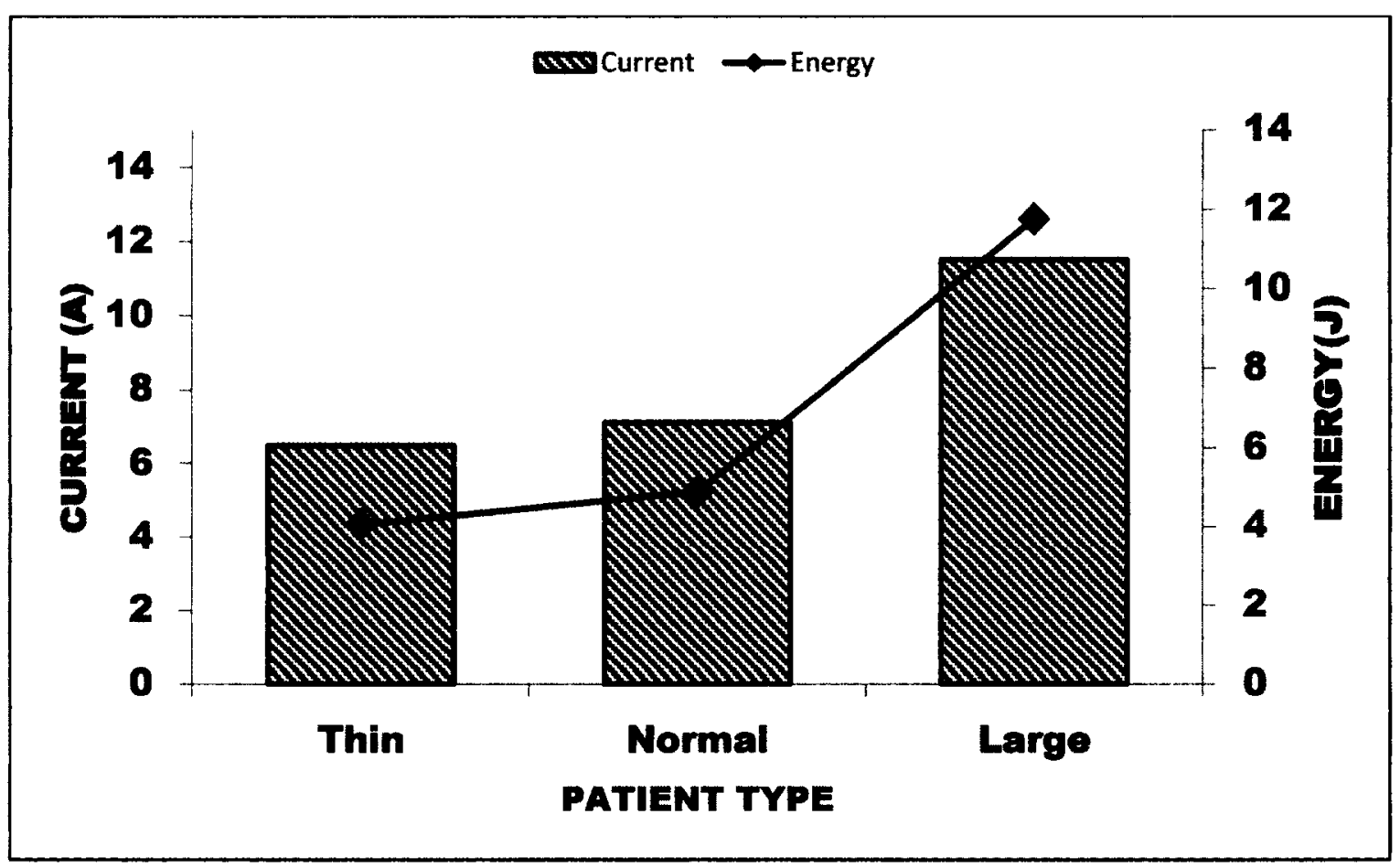

Figure 6.9 Minimum current and energy requirement for thin, normal, and large person. No cardiac damage was seen for all cases.

The resistance for the thin and normal patients were found to be similar, $43.74 \Omega$ and $43.95 \Omega$ respectively while the larger patient had a resistance of $42.18 \Omega$. This resistance is calculated when no fat tissues are assigned for a larger person. When we fill the large 
thoracic space with fat tissue, which is the case for the larger person, the resistance and energy are found to increase by $24 \%$ and $8 \%$ respectively. There is a decrease in the current requirement by $6 \%$, nonetheless the requirement is significantly higher than for the other two sizes.

Table 6.3 Difference in the defibrillation parameters in larger person with and without fat

\begin{tabular}{|c|c|c|c|c|}
\hline Large Size & $\begin{array}{c}\text { Mean current } \\
\text { density } \\
\left(\mathbf{m A} / \mathbf{m m}^{2}\right)\end{array}$ & $\begin{array}{c}\text { Minimum } \\
\text { current } \\
\left(\mathbf{m A} / \mathbf{m m}^{2}\right)\end{array}$ & $\begin{array}{c}\text { Energy } \\
(\mathbf{J})\end{array}$ & $\begin{array}{c}\text { Resistance } \\
(\mathbf{\Omega})\end{array}$ \\
\hline With Fat & 0.8494 & 10.8 & 14.61 & 50.04 \\
\hline
\end{tabular}

\subsubsection{Discussion}

It is intuitive that the current distribution in the thorax varies according to the shape and size of individuals. This work has been designed to investigate this difference, where it can be seen from Figure 6.7 that the mean current density in the heart is approximately $32 \%$ less in large person and $16 \%$ more in thin person than a normal sized person.

When a current level of $25 \mathrm{~A}$ is simulated, more damage in the cardiac tissue is seen for a thin size, which suggests that while delivering shock under real conditions, it would be advisable to start with a lower current (or lower energy) to avoid any cardiac tissue damage for thinner patients.

The minimum energy and current required for successful defibrillation is found to be $17 \%$ and $9 \%$ lower for a thinner person with respect to a normal sized patient. For a large size patient, the energy requirement is almost three times higher with $62 \%$ more current required for defibrillation. Even while modelling $20 \mathrm{~mm}$ thick fat tissue in the thorax, these values do not change drastically (Table 6.2). 
Such a difference in the values between the sizes is suggestive from the variation in thoracic circumference and thoracic space (Figure 6.6). In a thin person, the thoracic circumference is the least when compared to the other sizes. This results in a narrower thoracic space between the surface and the organs (lungs and heart). Though this thoracic space is conductive soft tissue, the current when delivered takes the shortest route via the lungs and heart, as seen in Figure 6.6 , rather than passing along the narrower and longer pathway of the chest wall. As a result, we can see large current influx in the heart than in any other region.

The same observation is made for a normal person. However, the current density in the heart is slightly less than that of the thin person. This could be due to an increase in the circumference which causes the current to get diverted along the conductive chest wall as compared to what it would be for a thin person.

For a larger person, the circumference is significantly larger, therefore the thoracic space is also larger. With a broader conductive pathway, most of the current seems to follow the extra cardiac pathways along the thorax wall rather than traversing through the lungs and heart, resulting in lower current density in the heart in this case. When this large space is filled with soft tissue, there is less resistance offered to the current flow, which explains the lower resistance for the larger person. However, the difference in resistance between large and normal/thin is small (only $1 \Omega$ ) since the distance between the electrodes has significantly increased due to the increase in thorax diameter.

As previously mentioned, in a human body the larger thoracic space is a result of deposition of the fatty tissue. When we add sub-cutaneous fat tissue along the thorax wall, we see a significantly increased potential difference between the electrodes and resistance (by $24 \%$ ) 
which result in an increased energy requirement. Nonetheless, the current density in the heart has reduced, but only by $5 \%$ indicating that the current still traverses more through the thorax wall than through the heart.

\subsubsection{Conclusion}

The three models representing three different healthy patients of same age, gender and height but different weight and BMI had distinct current distribution in the body with noteworthy variation in the values of the defibrillation parameters.

While the patient having larger BMI and weight required more current and energy for defibrillation, the patient with the lowest BMI and weight needed the lowest amplitude of shock. One of the primary reasons for such a result is the variation in the thoracic diameter between the patients.

The deposition of fat in a larger sized person results in enlargement of the thoracic diameter, thus giving more room for the current to flow along the chest wall. This not only increases the distance for the current to travel, which exacerbates the total body impedance but also reduces the current density in the heart.

This suggests that when a larger patient undergoes defibrillation, there is indeed a reduction in the current reaching the heart which ultimately results in lower resuscitation success. On the other hand, for a thin sized person, high current and energy shocks should be avoided to reduce chances of cardiac damage unless fibrillation have occurred for a longer period of time. 


\subsection{Effect of pad positions}

The results in section 6.3 imply that larger size patients exhibiting high thoracic impedance have a smaller current density in the heart, therefore more current and energy is required to achieve a successful defibrillation. Given the limitation of the biphasic defibrillators to deliver current and energy up to $27 \mathrm{~A}$ and $200 \mathrm{~J}$ respectively for a patient having a thoracic impedance higher than the average $(70 \Omega)$, it is important to reduce impedance in such larger patients. Among the many factors that influence the impedance, we have studied different positions for the pads used during cardioversion and have examined the position that gives the lowest resistance, lowest current and energy requirement, and yields the highest success.

This will not only act as a way to improve the performance of cardioversion for higher impedance patients, but will also help to reduce the uncertainty that exists in the defibrillation field regarding the most optimal pad position.

\subsubsection{Modelling}

Three pad positions (Figure 6.10) which are predominantly used for cardioversion are AnteroLateral (AL), Antero-Posterior 1 (AP1), and Antero-Posterior 2 (AP2). In AL position, one electrode is placed at the right sternum and the second electrode is placed on the apex of the heart. In AP1 position, the electrodes are placed at the anterior and posterior side towards the middle of the thorax. The AP2 position is similar to AP1, only the posterior electrode is placed towards the left of the thoracic cavity. 


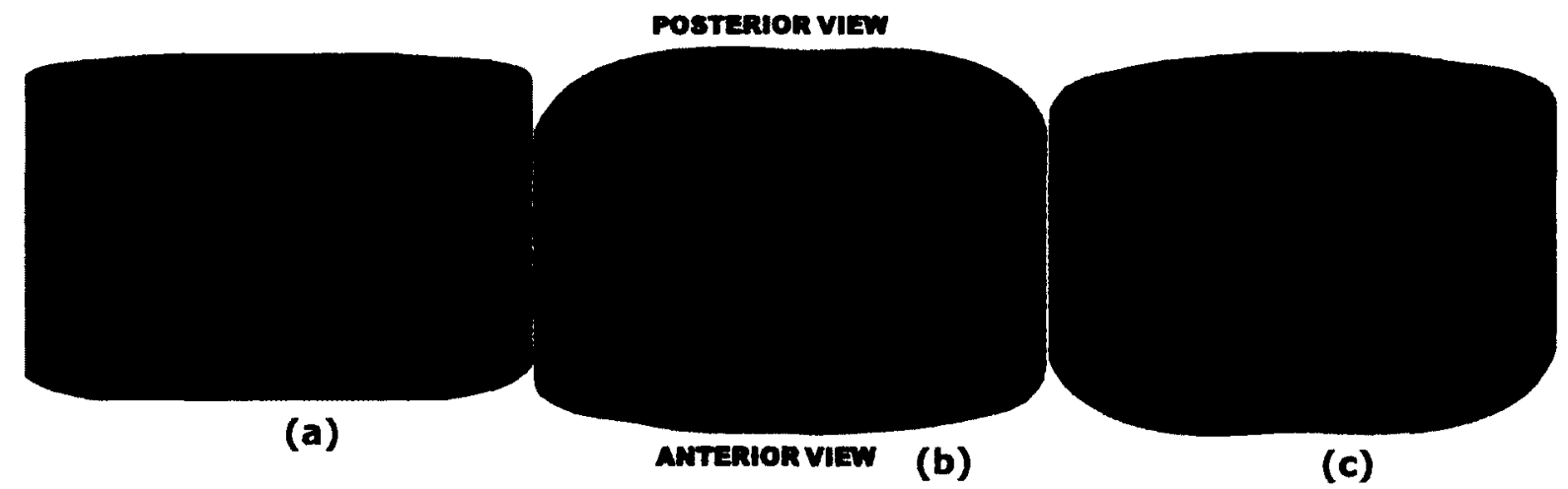

Figure 6.10 a. AL, b. AP1, and c. AP2

These three electrode positions were modelled on the FEM model generated from the T7 slice of a CT scan of a healthy and normal sized person, (Figure 6.2 and Figure 6.11). These positions were compared in terms of the defibrillation parameters mentioned in Section 6.2.

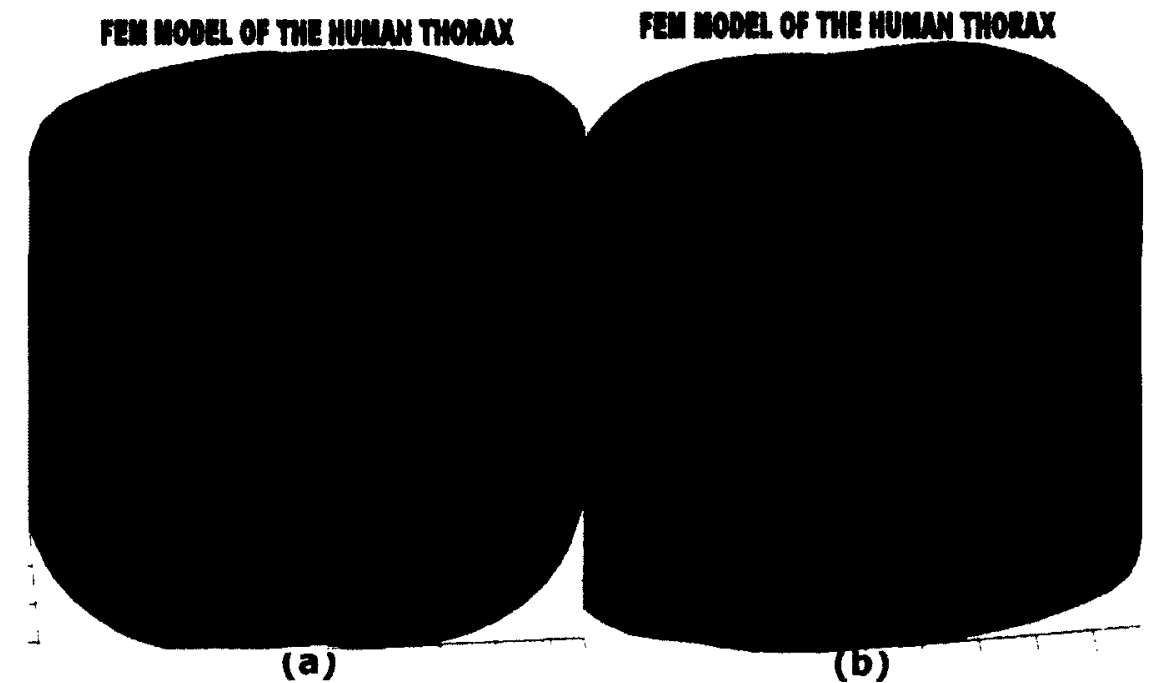

Figure 6.11 3-D Human Thorax Model in AL position: a. Anterior view, b. Posterior Side (Right). Dark Blue represents Ribs and spine, Red-Heart and Blue- Lungs

In a clinical scenario, a physician need not always place the electrodes exactly in the positions we have modelled. There would be slight shift in the position either to left, right, upwards, or downwards. Considering this variation, we shifted the electrodes $20 \mathrm{~mm}$ left, right, up, and down from their original location, shown in Figure 6.12. This resulted in 5 shifts for each electrode and since there were two electrodes, we got a total of 25 different placements in the electrode for each of the AL, AP1 and AP2 position. The defibrillation 
parameters were calculated for these 25 values which were then used for statistical and analytical comparisons of the electrode positions.

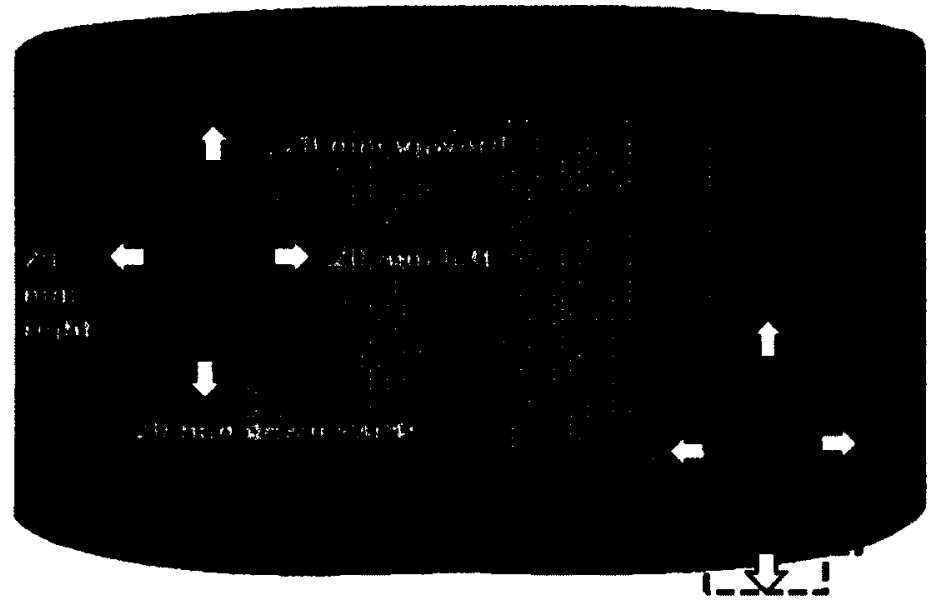

Figure 6.12 Shifts in the electrode positions.

\subsubsection{Statistical Analysis}

The minimum current and energy required for successful resuscitation, resistance, and HI for the three positions were compared using one-way Analysis of Variance (ANOVA). Our hypothesis is that there is no significant difference between the three positions for all these parameters. If the results are statistically significant, post-hoc Tukey's Honestly Significant Difference (HSD) test is performed for pairwise comparison of positions. All the statistical testing was evaluated at a significance level of $\alpha=0.05$.

\subsubsection{Results of pad position}

Figure 6.13 shows the current density and voltage distribution in the thorax for all the three positions. The mean current density in the heart is shown in Figure 6.13 with AL showing $0.6100 \mathrm{~mA} / \mathrm{mm}^{2}, \mathrm{AP} 10.5498 \mathrm{~mA} / \mathrm{mm}^{2}$, and AP2 $0.5788 \mathrm{~mA} / \mathrm{mm}^{2}$.

It was found that minimum and maximum current density were respectively higher than $J_{\mathrm{th}}$ and lower than $\mathrm{J}_{\max }$ which implies that entire heart was defibrillated without causing any damage to the cardiac tissue. 


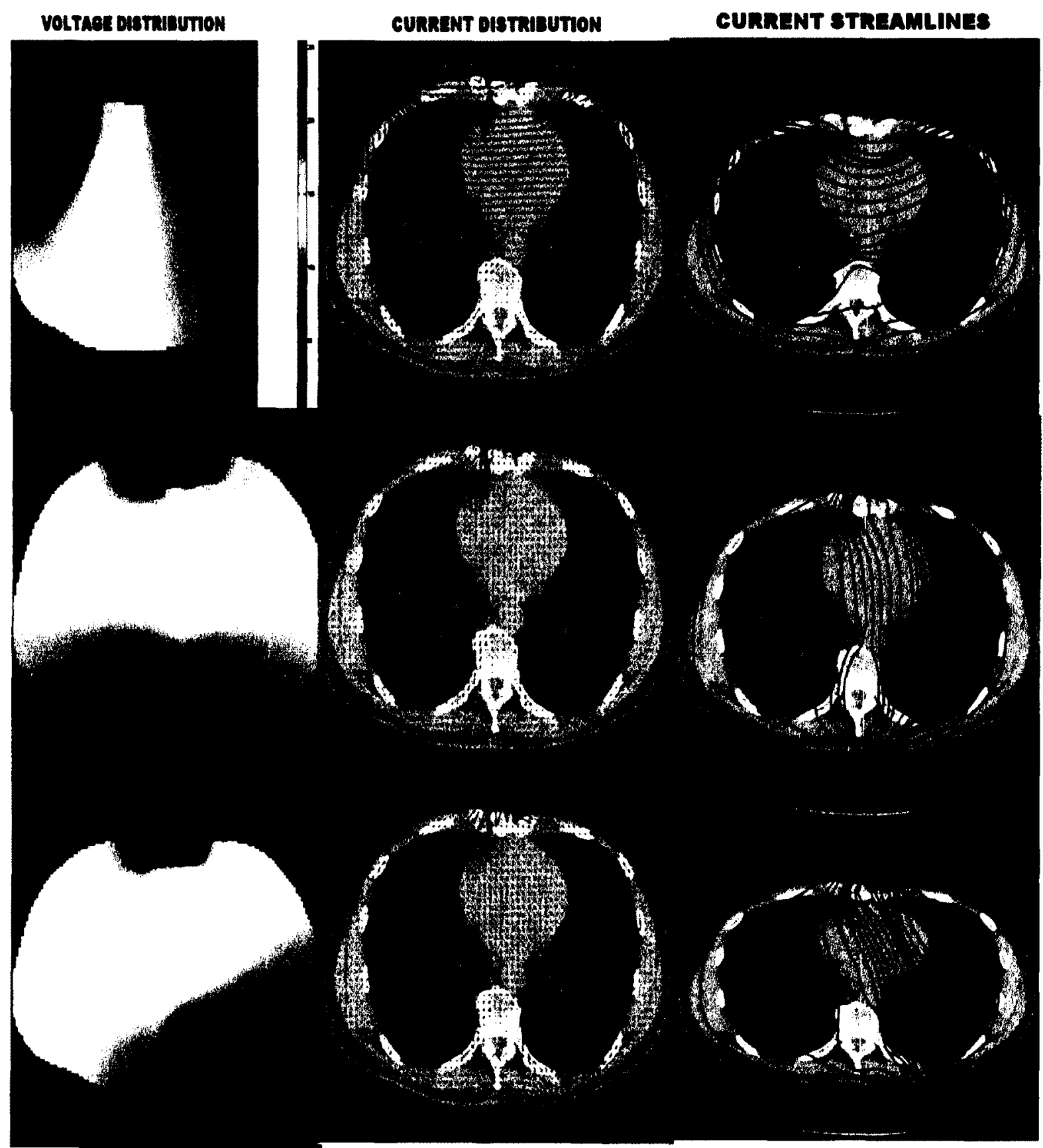

Figure 6.13 Voltage and Current distribution for a. AL (TOp), b. AP1 (Middle), and c. AP2 position (Bottom) 


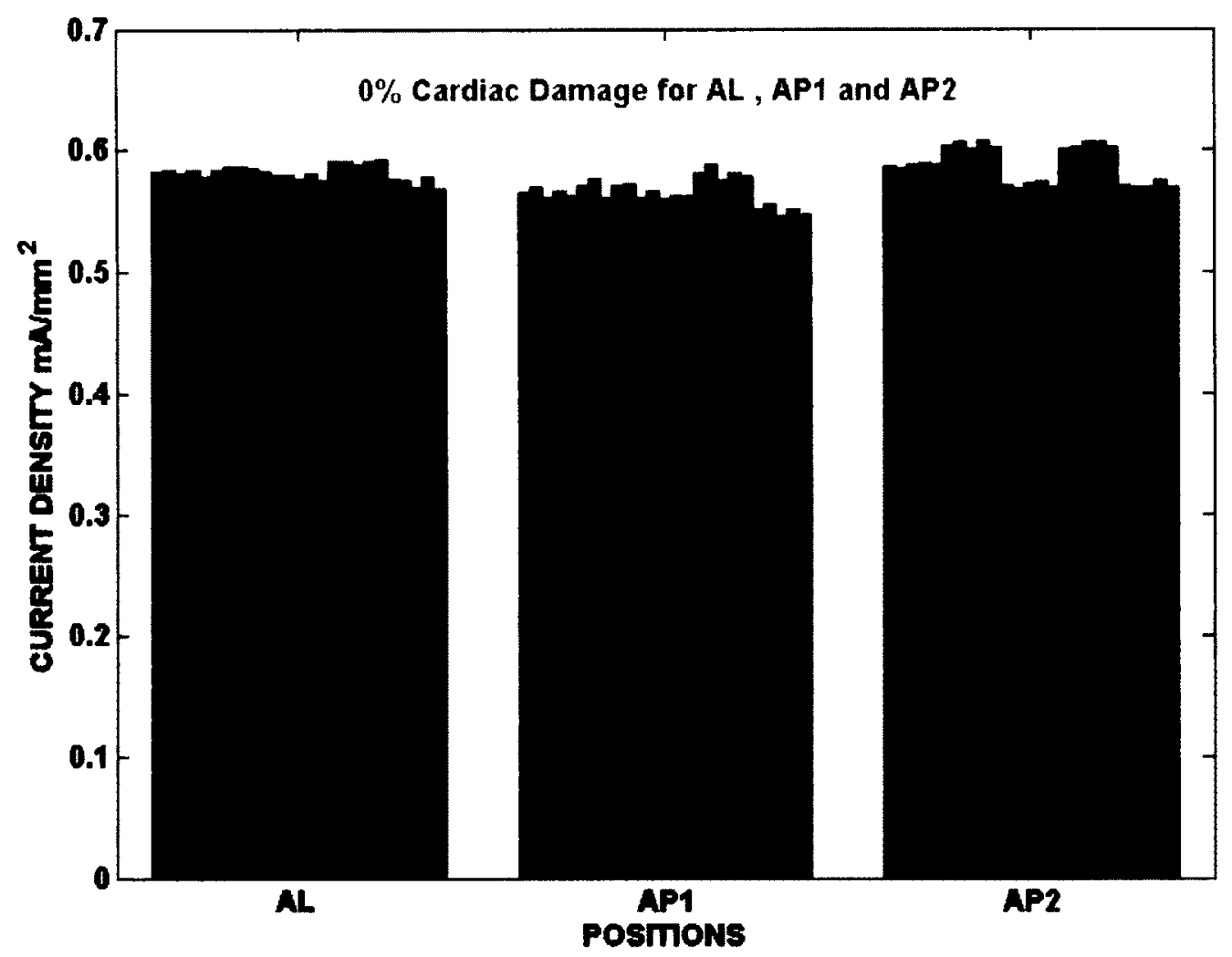

Flgure 6.14 Mean Current Density in heart for AL, AP1, and AP2 positions. The 25 bars in each positions shows the mean current density when the location is changed to $20 \mathrm{~mm}$ left, right, up and bottom.

The $\mathrm{HI}$ is largest for $\mathrm{AL}$ with a value of 0.872 indicating a higher non-uniformity in the current distribution as compared to AP1 and AP2 which were 0.5600 , and 0.470 respectively (Figure 6.15).

The minimum current required to defibrillate $95 \%$ of the heart is lowest for AP2, as shown in Figure 6.16 and highest for AL. Calculating defibrillation energy threshold, it was seen that AL requires $53.72 \mathrm{~J}$ to defibrillate which is $59 \%$ more than AP1 which requires $33.59 \mathrm{~J}$ and $86 \%$ more than AP2 which requires $28.78 \mathrm{~J}$. 


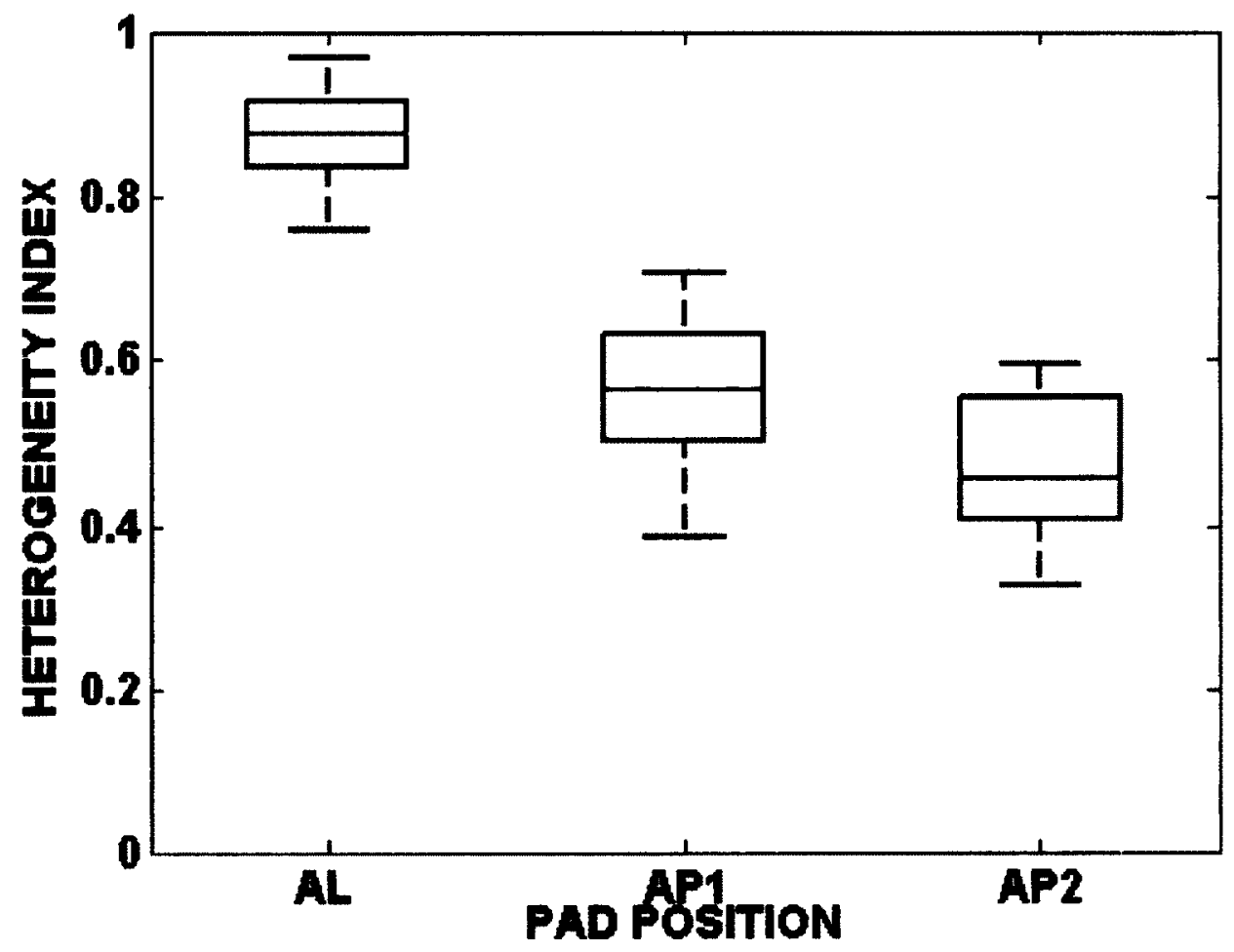

Figure 6.15 Box plot showing variation in Heterogeneity Index (HI)between the three positions with their shifts in the locations

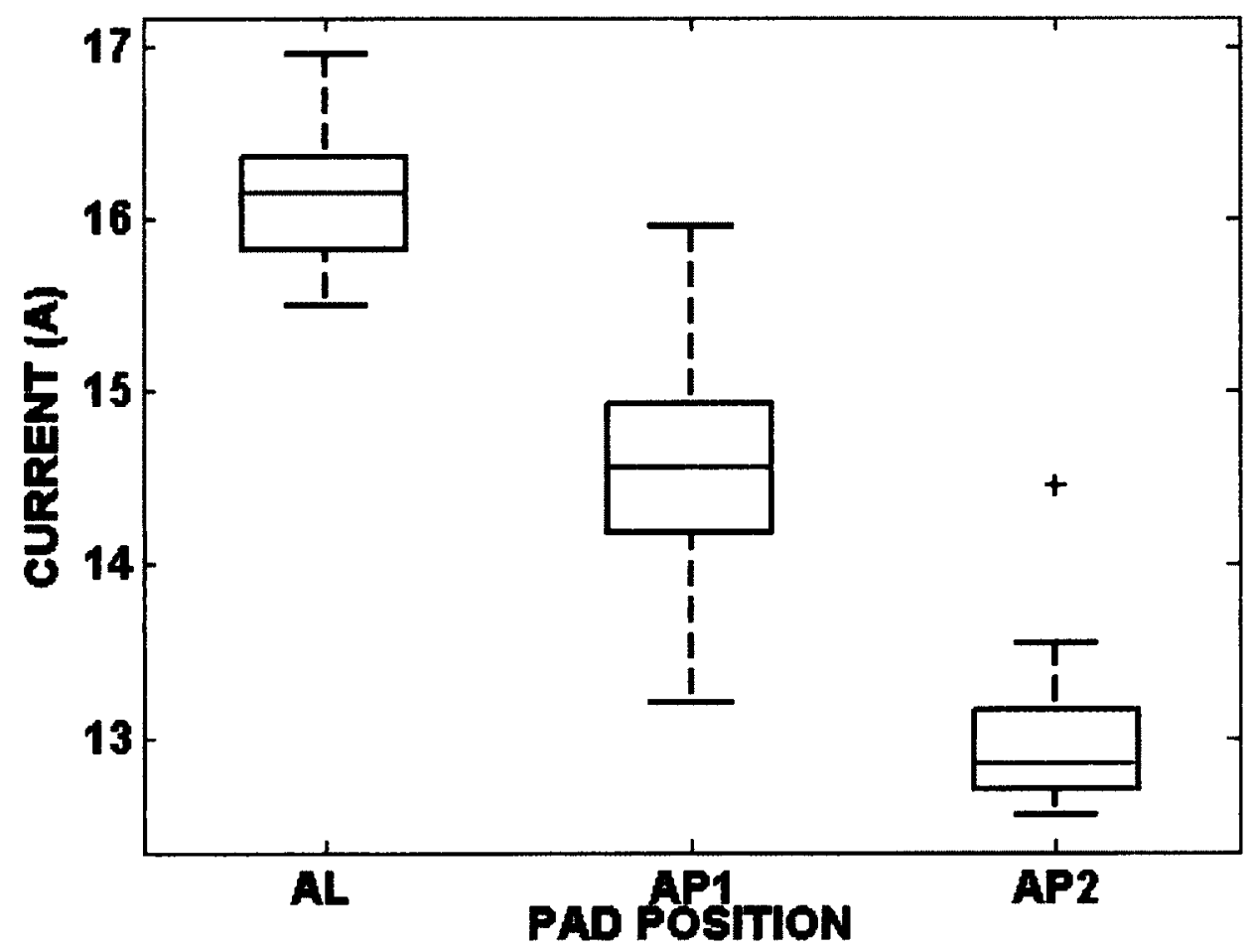

Figure 6.16 Variation in current requirement within and between the three positions 


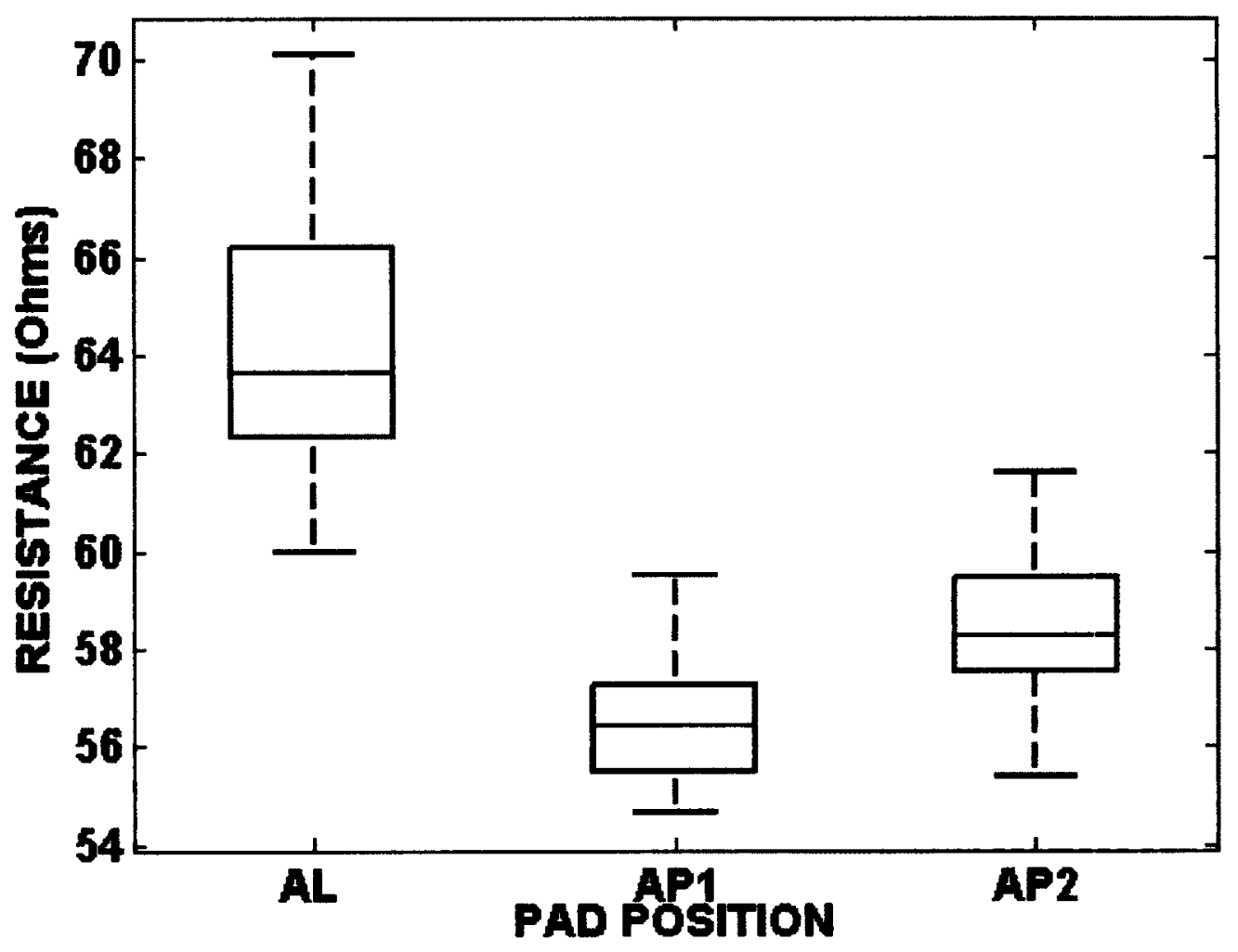

Figure 6.17 Comparison of pad positions with respect to resistance

Figure 6.17 shows the comparison in the resistance value measured for the three positions. The highest resistance was observed for AL $(64 \Omega)$ and the lowest for AP1 (56 $\Omega$ ). We can also see that the resistance offered by AP2 (58 $\Omega$ ) is very close to the resistance in AP1.

We also correlated $\mathrm{HI}$ and energy required for minimum defibrillation. Linear regression and Pearson correlation coefficient was performed to determine the relationship between Energy and Heterogeneity Index. The correlation coefficient was calculated to be 0.92 and $R^{2}$ as 0.86 . This suggest a strong positive correlation between these two parameters. 


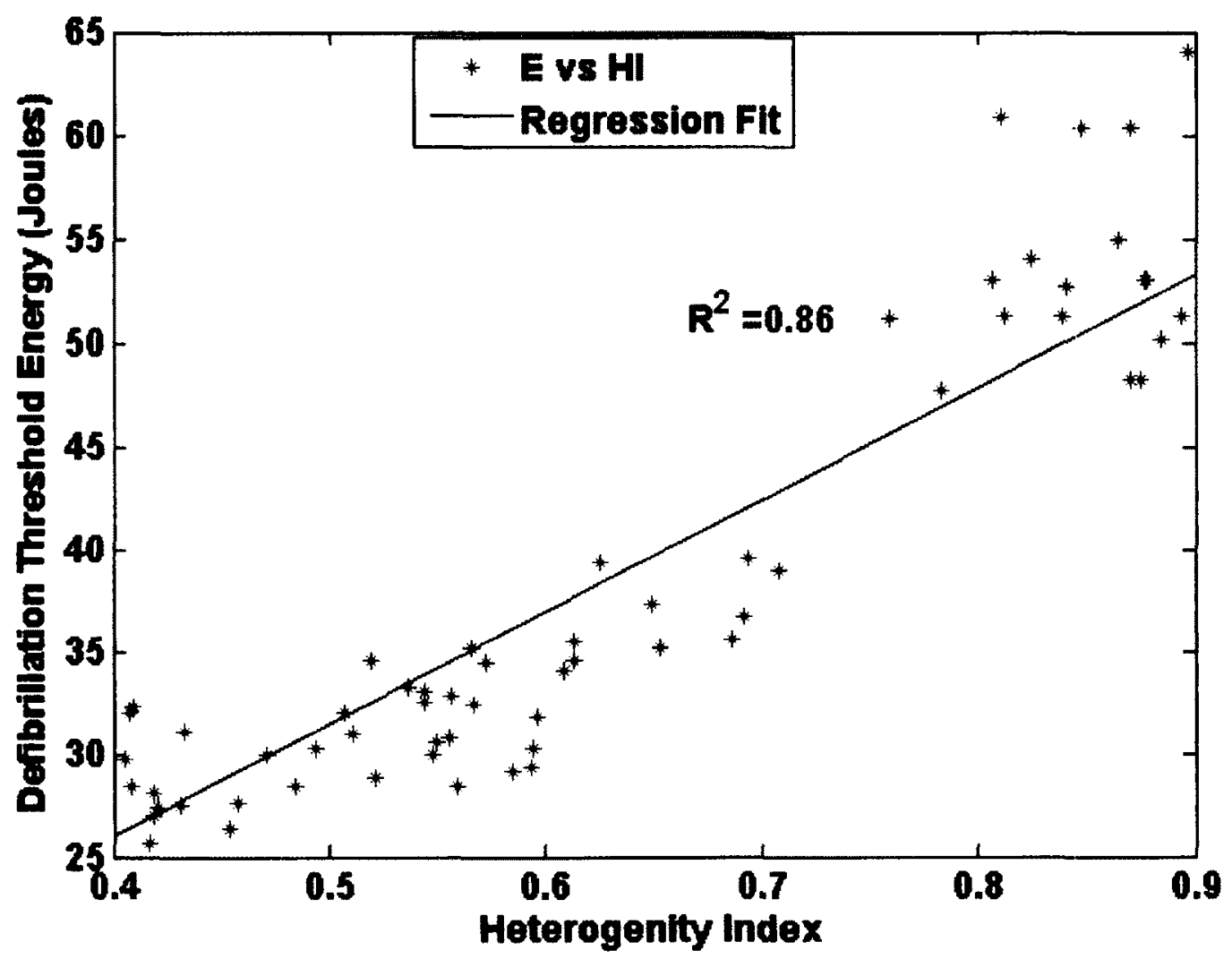

Figure 6.18 Correlation between Heterogeneity Index and Energy

The comparison results were then statistically compared using one-way ANOVA, as shown in Table 6.4. Since p-values for all the three dependent variables is less than 0.05 , null hypotheses mentioned in section 6.4 .2 is rejected which suggests that significant difference exists between these three positions with respect to all the three dependent variables ( $p<0.05$ for all the three variables). Since ANOVA does not tell us between which pair the difference exists, we performed Tukey's HSD test, Table 6.5. The results suggest that a significant difference exists in the defibrillation parameters between the three positions.

Table 6.4 Results of one-way ANOVA

\begin{tabular}{|c|c|c|c|}
\hline & $\begin{array}{c}\text { Current reqd. to defibrillate } \\
95 \% \text { of heart }\end{array}$ & HI & Resistance \\
\hline 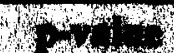 & $\therefore(0.0000)$ & (0.)(0) & $x(x) 1$ \\
\hline
\end{tabular}


Table 6.5 Results of Tukey's HSD test

\begin{tabular}{|c|c|c|}
\hline Positions & Dependent variable & p-value \\
\hline 67. & 36) & 0 \\
\hline AL-AP2 & & 0 \\
\hline 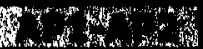 & 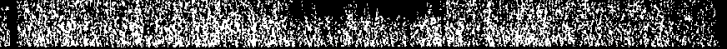 & 0 \\
\hline AL-AP1 & \multirow{3}{*}{ Heterogeneity Index } & 0 \\
\hline Why & & 0 \\
\hline AP1-AP2 & & 0.001 \\
\hline (2) & 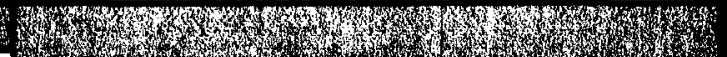 & 0 \\
\hline AL-AP2 & (1) & 0 \\
\hline 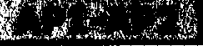 & 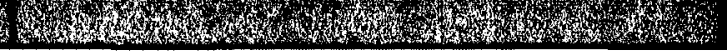 & 1) \\
\hline
\end{tabular}

\subsubsection{Discussion}

The results of this study suggest that each of the three pad positions that were investigated could defibrillate the entire myocardium when stimulated with $25 \mathrm{~A}$ current amplitude. It was noted that AL position offers the highest range for current density (Jmax - Jmin). This indicated that in AL position, some portion of the heart receives a very high current and some very low. This is more evident from Figure 6.13 , where we can see that the current pathways are more concentrated along the thorax wall and the ventricles than the rest of the myocardium. One plausible explanation for such an observation could be the placement of electrodes. The electrodes are placed in such a way that the current can complete its path via the conductive tissues of the heart and the chest wall rather than traversing through other portion of the heart that is surrounded by a very resistive lung region. For the AP positions, it can be seen that current streamlines traverse well within the heart, especially in case of AP2, as shown in Figure 6.13. Also, there is no large difference in the values of current density for shifts in the electrode location for all the three positions, which indicates that a change in the pad position by a few millimeters does not produce a significant variation in the current density in the heart.

In terms of uniformity in current distribution, AP2 offers more uniform conduction as compared to AL and AP1 since it exhibits lower HI. Calculating the HI for the intra variation 
in electrode position, it can be seen from Figure 6.15 that variation in $\mathrm{HI}$ for $\mathrm{AL}$ is not large as the width of the box (inter-quartile range) is fairly small; for AP1 and AP2, the interquartile range is large and the whiskers are away from the upper and lower quartile range indicating that the some of the values for these case are varying largely with the change in the electrode placement. The upper whisker in AP1 towards the range of 0.7 is the result of the pad shift towards the right and the lower whisker below towards the range of 0.3 to 0.4 as a result of shift towards the left. The same observation was seen in AP2 where maximum $\mathrm{HI}$ was seen in shift towards the right and lowest towards the left which has made the max and min deviate largely from the median value. Since the heart is located slightly towards the left in the thoracic cavity, the change in the position of the electrode towards the right (slightly away from heart) results in more non-uniformity in the distribution and towards left results in more homogenous conduction and higher mean current density as well. These results indicate that the current uniformity in the heart is very sensitive to placement errors. In a clinical scenario, such electrode shifts are prone to occur by human operators. Therefore, the pads have to be cautiously applied on the chest and care must be taken to not place the electrodes further right from the ideal position.

There also exists strong positive correlation between $\mathrm{HI}$ and energy. From our data, we found that the more uniform a distribution is, the less energy will be required for defibrillation. AP2 which offers highest uniformity defibrillates the heart with energy $86 \%$ and $18 \%$ lower than $\mathrm{AL}$ and $\mathrm{AP1}$. This observation is also consistent with the results shown in $[49-52]$.

Though AL position shows the highest mean current density, it is the AP2 position that best fulfills the requirement of successful defibrillation with the least current. AP2 has been found to offer more uniformity, lesser energy and current than the other two positions. The electrodes in AP2 are placed at the left side of the posterior position. This position matches 
with the orientation of the heart, which is also situated towards the left, there by defibrillating more volume of the heart than the other two positions.

With the shifts in the electrode position, there is no large variation in the current requirement for $\mathrm{AL}$ as the width of the box plot is small and the distance of the minimum and maximum value from the box is not large. The whiskers in AP1 and the outlier in AP2 show variation in current within these positions. These large variations were seen only when the electrodes were shifted away from the heart towards right and upward direction.

Comparing these three positions in terms of resistance, AP1 is found to offer a lower resistance pathway than AP2 or AL. The path taken by the current in AP1 position is via soft tissue, bones and heart. In $\mathrm{AL}$, the current takes the path of the lungs, bone, chest wall, and with AP2 bone, heart, a lung and connective tissue. As a result the potential drop is smaller for AP1, as compared to AL or AP2. However, the box plot in Figure 6.16 also shows that the resistance value in $A P 1$ and $A L$ is more prone to vary with the shift within the placement of the pads.

\subsubsection{Summary}

The results in this study suggest that electrode position is an important factor for a successful defibrillation.

Statistical analysis conducted shows significant difference in the values of the defibrillation parameters yielded by these positions. This suggests that electrode position makes a major difference in defibrillation, contradicting the results in [2][7][53][86] which claims that electrode position makes no difference in cardioversion. 
The results also indicate that the defibrillation parameters can be affected by the placement errors especially towards the shifts in the right position. Comparing the three pad positions, the standard AL position has been found to be not as effective for cardioversion as AP. There is more current flowing towards the ventricular region for $\mathrm{AL}$ with $50 \%$ lesser conduction uniformity and $40 \%$ more defibrillation energy than AP positions.

Comparing the two AP positions, the current density values are very close to one another; however, in terms of homogeneity, resistance and minimum energy required for defibrillation, AP2 is found to be more suitable during cardioversion. This result is in consistent with the results shown in [50][53][57]. Work by Botto et al. on clinical data showed that lower DC energy were required to defibrillate patients in AP position (which is AP2 for our case) with a higher success rate than AL position. Hunt et al. showed similar results for AP2 using FEM where this position required lesser current and energy for a successful defibrillation.

\subsection{Pad position and sizes}

With AP2 position proving to be more efficacious for cardioversion, we also tried to confirm the results by examining all three positions on the different patient sizes that we considered for the analysis in section 6.3.

As we can note from Figure 6.19 , the amount of current density in the heart for a larger person seems to be the highest for AP2 position. For normal and thin sizes, the current density for AP1 and AP2 are relatively similar to each other and AL shows the lowest current density. 


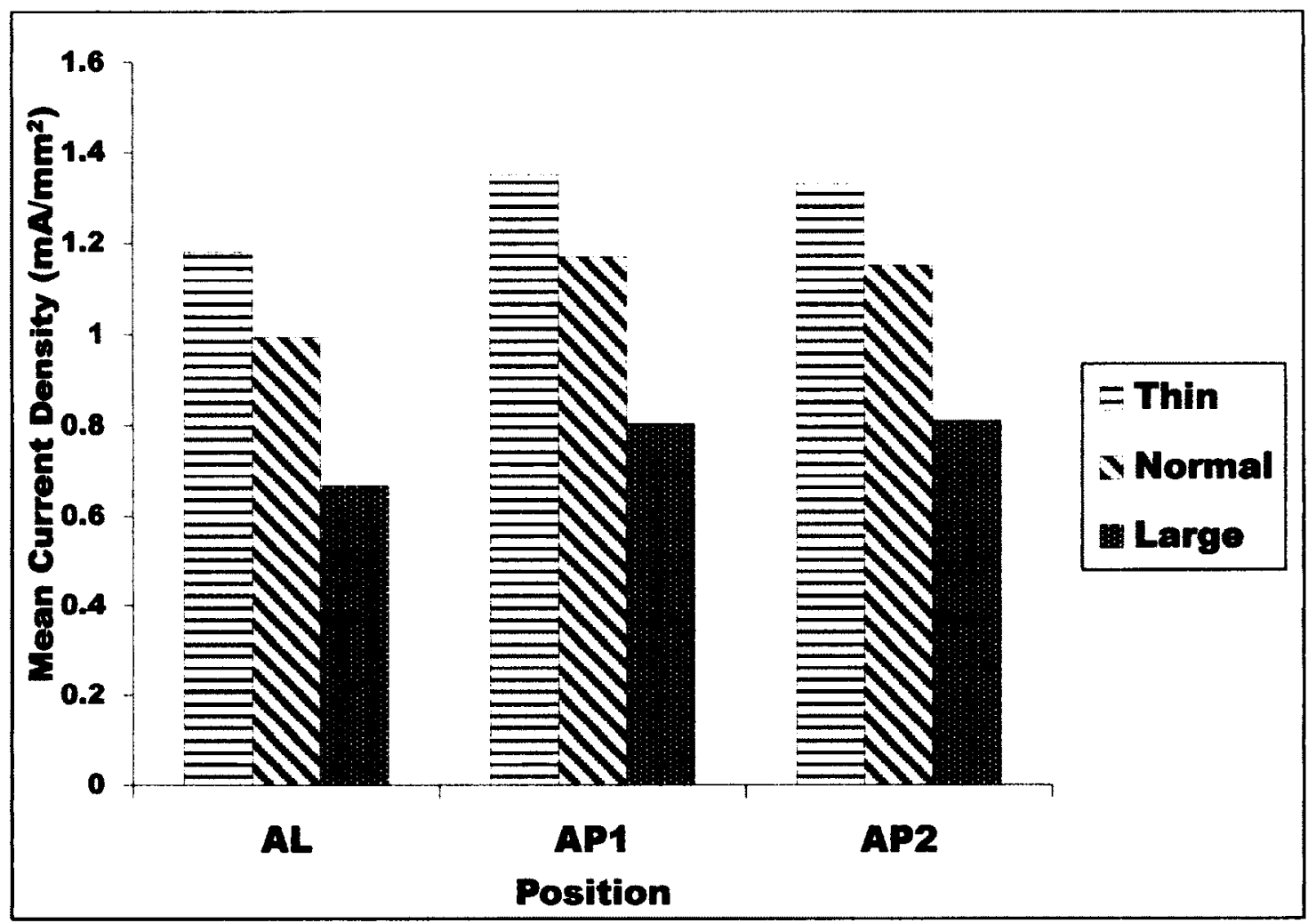

Figure 6.19 Mean Current Density for different sizes and positions

The current and energy required to achieve $95 \%$ of depolarisation in the cells for AP2 on a larger person is similar to the requirement for $\mathrm{AL}$ on a thin and normal person (Figure 6.20). 


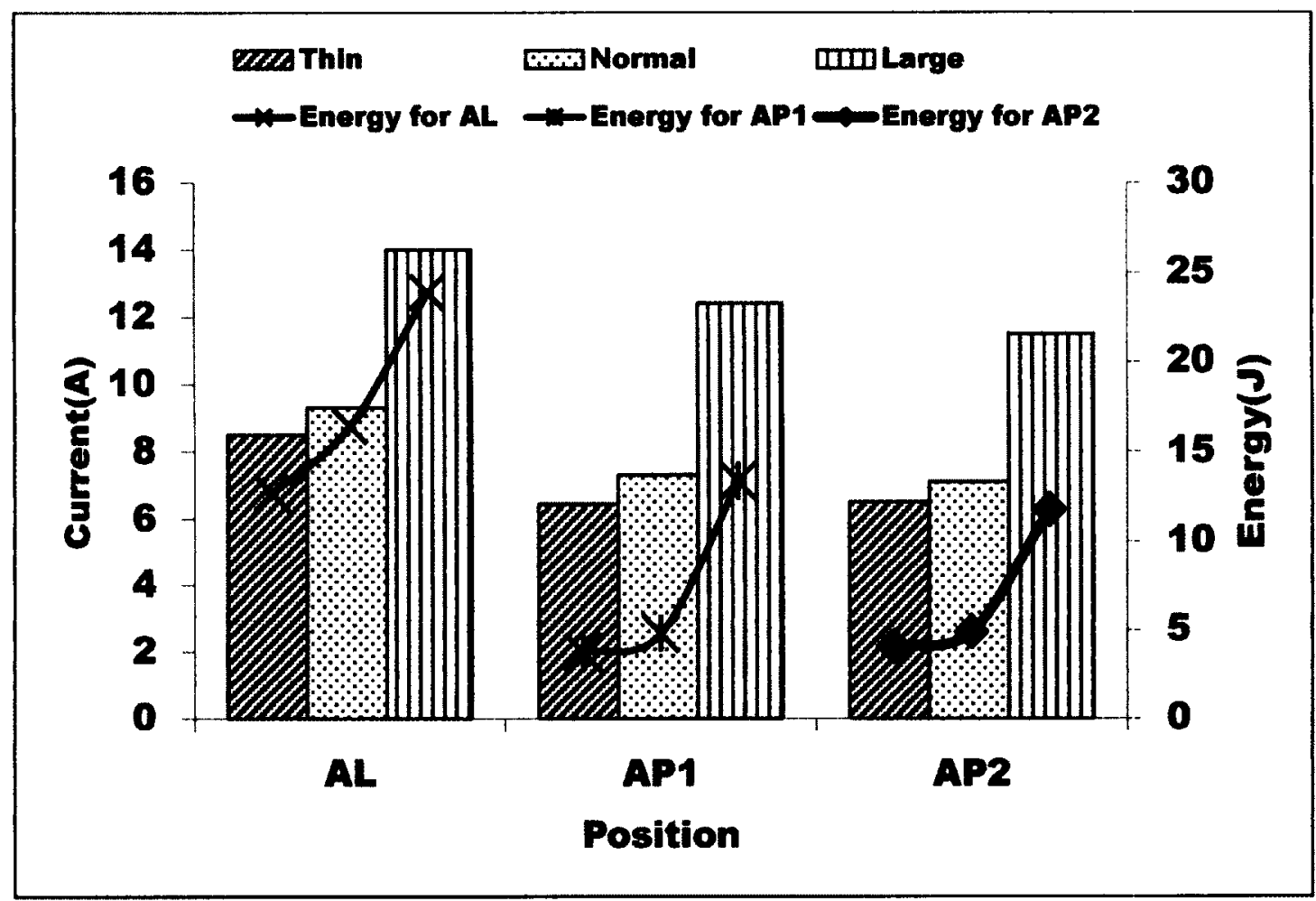

Figure 6.20 Current and energy requirement for different pad position and sizes

These results indicate that placing the electrodes in AP2 position is an optimal position which can largely improve the success rate for patients showing higher impedance.

\subsection{Limitations}

Our FEM model has attempted to match a real cardioversion procedure including the electrode shape, size, and position that is used clinically. It also includes the calculation of different defibrillation parameters (section 6.2) and has graphically shown the voltage and current density distribution in the thorax during defibrillation which is a vast improvement over the models shown in the previous FEM studies.

However, it is limited by the accuracy of the tissue conductivities and anisotropic properties. The true anisotropic representation of the human thorax may alter the results. The model also does not account for factors such as patient's history of arrhythmia, effect of antiarrhythmia treatment, duration of arrhythmia, temporal variation of biphasic waveform, 
which in a clinical scenario affects the cardioversion efficacy. Also, the symmetry of the body along the chest may not be present in larger patients due to irregular deposition of fat in the thorax. Nevertheless, our FEM model gives a fairly good approximation for the electrical activity in the thorax.

\subsection{Conclusions}

This chapter presented a model of the human thorax using FEM and measured different defibrillation parameters to investigate the effect of size and pad position during cardioversion.

When the same current amplitude was applied to individuals with differently-sized thoraxes exhibiting different impedance values, larger patients showed less current in the heart and a higher requirement of current and energy to achieve successful defibrillation. This underscores the influence of thoracic geometry on the body impedance which ultimately affects the current reaching the heart.

Among the three pad positions, AL, AP1 and AP2, it was observed that AP2 position was more effective during cardioversion since it required less energy and current for defibrillation and offered more uniformity in the current distribution. The results also suggested that changing the electrode position in large impedance patients to AP2 would be more favorable for gaining successful results during cardioversion.

To summarize, the pathway the current takes for different sizes of patients and different pad positions result in variation in the defibrillation parameters which is significant enough to affect the performance of cardioversion. Since the current conduction is largely affected by the resistance offered to the current flow by different tissues, our results in this analysis suggests that resistance does affect the efficacy of cardioversion. 


\section{Thesis Summary and Future Recommendations}

\subsection{Summary of conclusions}

In this thesis, we have explored the effect of trans-thoracic impedance on cardioversion through statistical analysis and Finite Element Method (FEM) analysis. The results are summarized below for each investigation.

\section{Retrospective Statistical Analysis.}

The clinical data comprising of 775 cardioversion cases with total of 1253 shocks for atrial fibrillation (AF), atrial flutter (AFL) and ventricular tachycardia (VT) was evaluated for the relationship between impedance and success rate using two statistical tests, Chi-square tests and Fischer's exact test. This data was divided into two groups of impedance; less than or equal to $70 \Omega$ as low impedance group and greater than $70 \Omega$ as high impedance. Statistical tests were performed at significance level of $\alpha=0.05$. With TTI as an independent variable and success as a dependent variable, the results showed significant relationship between $\pi \mathrm{I}$ and success rate for AF $(p=0.04)$ and VT $(p=0.006)$. For cases representing AFL, the success rate was more than $90 \%$ for any given impedance and energy level, and the results were not significant $(p=0.11)$.

The statistical testings was also performed with energy as an independent variable and success rate as a dependent variable. Our statistics showed no difference in the results between the two energy levels on the success rate.

The peak current-success relationship that we have plotted in Chapter 4 indicates that delivering current between a range ( 28 to $48 \mathrm{~A}$ for AF and 28 to $32 \mathrm{~A}$ for VT) improves the efficacy of the treatment. Delivering current outside the given range 
causes a decline in the success rate. This optimal current and its relationship with shock success helps explain our results obtained from the statistical testing. The success rate was higher for low impedance and lower for high impedance for AF and $V T$ because of the delivery of current outside of the optimal current range. The success rate was not affected between the energy levels for the same reasons.

\section{FEM Modeling.}

A 3-D model of the human thorax was constructed using FEM in order to study the distribution of current in the heart and the thorax. The defibrillation parameters such as current density in the heart, uniformity in the current distribution, minimum current, and energy required for a successful defibrillation, and potential damage to the cardiac tissues were measured. These parameters, which are considered to be an indicator of the success of defibrillation, were used to compare the cardioversion results in different sizes of the patients and also different pad positions. It was found that a lesser amount of current and energy were required to attain a successful defibrillation in a thinner patient having lower impedance as compared to larger person exhibiting higher impedance. Since more current reaches the heart for a thinner patient, more predicted cardiac damage was observed in this case and no damage was seen for a larger person.

Among the three pad positions that are predominantly used during cardioversion, $\mathrm{AL}$, AP1 and AP2, it was found that AP2 position was more effective since it required lesser energy and current for a successful defibrillation and offered more uniformity in the current distribution. Our results also suggested that the defibrillation parameters can be sensitive to placement errors, especially if the shift in the pad is towards the right. 
When different positions were tested on patient sizes, the results suggested that changing the electrode position in large impedance patients to AP2 would be more favorable for gaining successful results.

The course of current in the thoracic cavity varied according to the sizes and positions which resulted in the variation of the defibrillation parameters for each of these cases.

The overall conclusion that can be drawn from this work is that impedance affects the performance of cardioversion, with success rate and $T I I$ being inversely related to each other. One of the ways to clinically increase the success rate of the treatment could be to cardiovert the patients in Antero-Posterior position.

\subsection{Recommendations for future work}

There are number of significant works that can be undertaken to better understand and improve the defibrillation treatment.

1. Prospective Study: Our work focuses on retrospective analysis on clinical data to explore the effect of TII on cardioversion success rate. One of the directions that the future work could take is on prospective data analysis. This would help measure other important statistics (like duration of arrhythmia or pathological condition of the patient's heart) that could influence the efficacy of cardioversion. The prospective study can also include clinical evaluation of the pad position. This would help affirm the result that has been seen in this work for pad positions using FEM.

2. Current based defibrillators: It is well known that electrical current is a precise indicator of defibrillation success. However, defibrillators are based on the amount of energy delivered. The impedance compensation of these devices therefore deliver same amount of energy by increasing the duration and not the magnitude of the 
current. This has led to impedance still affecting the success of cardioversion (Refer to Chapter 4). Future work of this thesis could include developing a prototype for current based defibrillators, where the descriptor of defibrillation threshold is current and not energy. This could be further supplemented by impedance compensation phenomena with the magnitude of current being adjusted according to the impedance of the patient. The current based defibrillators could be helpful in reducing the impact of impedance and increasing performance of the defibrillation treatment.

3. Measurement of the impedance before the cardioversion procedure: Our results define the impact of impedance on a cardioversion treatment. Measuring and displaying $\pi T 1$ to the clinicians prior to shock application will allow the clinicians to take measures to reduce the impedance. The biphasic defibrillators today, though compensates for the impedance, however the value of impedance is not displayed in the device during the cardioversion procedure. This limits the clinicians to use the same energy delivery protocol regardless of $\pi \mathrm{TI}$, which results in more number of shocks to defibrillate high impedance patient. Knowledge of the impedance before the delivery of the shock may allow the clinicians to start with a higher energy level for high impedance patients, and lower the energy level for low impedance to avoid any tissue damage. This approach may not only increase the success rate of cardioversion but also increase the safety of this treatment.

4. Refining the FEM model: We have made a sincere effort in trying to match the FEM model to the real human thorax and obtain a good approximation for the electrical activity in the body. However, the model has its limitations (as mentioned in section 6.6 of Chapter 6) and more work is required to improve the capabilities of the existing FEM model. 
In our work, only the contours of lungs and thorax wall are given to the NETGEN for the finite element mesh generation. The heart, bony structures and fat tissues are then manually modelled based on the dimension of these tissues in the CAT scan. The future work for this study could include developing algorithm so that NETGEN takes into account all these structures and generates a model that accurately represents the human body.

Our assumption regarding the translational symmetricity of the body along the chest might not hold for large patients due to an uneven deposition of fat in the body. One of the potential areas for further research could be to develop an anatomical 3-D model using all the 64 slices of the chest CAT scan. Anatomically detailed and accurate 3-D model can also be constructed using MRI images since this imaging technique provides higher resolution and more finer anatomical details than CAT scan.

The conductive properties of the tissues affect the electrical parameters. Therefore, the future work should incorporate modeling the anisotropic behaviour of the tissues for the electrical activity in the model to exactly represent the electrical behaviour in a real human body.

The defibrillators today deliver current that changes its direction and magnitude with time which is not how the current is simulated for our model. One of the areas for future work could be inclusion of this temporal variation of the current in our model. Overcoming these limitations in our model will help us accurately understand the electrical behaviour of defibrillation during its clinical use.

5. Analysis of pad shape and pad size: Our FEM model can be used towards examining different pad shapes, for e.g. rectangular, square, circular, and ellipsoidal 
along with different pad sizes that would be useful in obtaining higher cardioversion success.

6. Finding current in the heart: The idea to determine the amount of current reaching the heart has been preliminary explored. However, due to time constraints, its implementation could not be carried out in this work.

To calculate the current reaching the heart, we have to calculate the normal current in each of the surfaces of the elements that make up the boundary of the heart. The current in each element is calculated by multiplying the current density $J$ with the surface area of the element $d S$ having a normal unit vectorn. The current in each of the element is then summed to calculate the current in the heart, as shown below.

$$
I=\int_{S} \vec{J}_{e} \cdot \hat{n} d S
$$

where, I is the current in the heart, $\vec{J}_{e}$ is the normal current density in the element, $d S$ is the surface area and $\hat{n}$ is the unit vector.

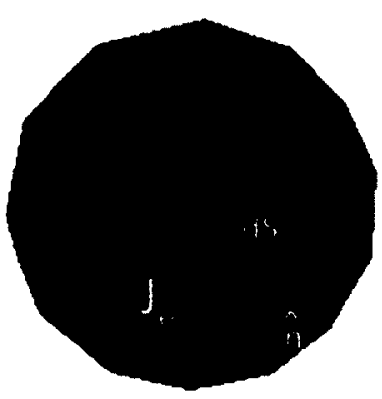

Figure 7.1 Calculation of current in an object. Blue represents a spherical ball and black is a boundary element.

7. Comparison of FEM results with the clinical data: The results of the defibrillation parameters obtained from the FEM could be compared with the clinical data to evaluate the model in terms of its clinical applicability. This would require construction of the thorax model for a patient who has had a previous CAT scan and 
will be undergoing cardioversion. Defibrillation parameters like energy and current required for successful defibrillation could be calculated using the methodology in this work. These values could be then compared with the clinical values obtained during cardioversion. This could also find its application in reverse engineering where using CAT scans of the patients and FEM, the best cardioversion scenario (pad position and defibrillation parameters) for the patients could be predicted retrospectively or prospectively to improve the success rate of this treatment.

8. Greater diversity in the subjects for examining the effect of $\mathrm{TTI}$ : In our thesis we considered only three FEM models, each for small, normal and large sized patient to explore the effect of TTI using FEM. The future work could include in-depth analysis of the effect of impedance by incorporating greater diversity of subjects in the small, normal and large body sizes.

9. Inclusion of Defibrillation events: Our analysis was focused only for cardioversion which is a non-emergency situation and the risk to the patients is less. We have not considered any defibrillation (ventricular fibrillation) cases which are more lifethreatening. These situations require $100 \%$ success rate at least within the first three shocks. Using the retrospective clinical data together with the FEM model could be an interesting way to explore ways to improve the success rate for this cardiac arrhythmia. 


\section{Appendix I: CLINICAL DATA}

Appendix I include a detailed description of the clinical data that we have acquired from UOHI. We have shown only 26 cases with a total of 50 shocks due to the length constraint of the thesis. The abbreviations used in the table are mentioned below:

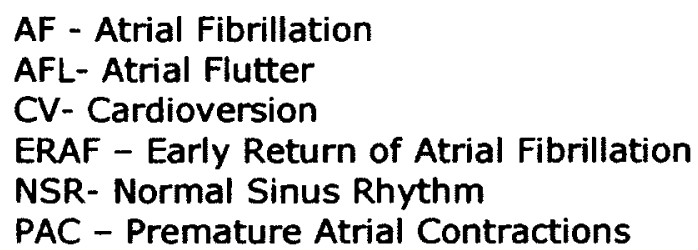

PAC - Premature Atrial Contractions

\begin{tabular}{|c|c|c|c|c|c|c|c|c|}
\hline Case number & Location & $\begin{array}{c}\text { Cardiac } \\
\text { Arrhythmia }\end{array}$ & $\begin{array}{l}\text { Details of } \\
\text { the } \\
\text { treatment }\end{array}$ & $\begin{array}{c}\text { Success/ } \\
\text { Failure }\end{array}$ & $\begin{array}{l}\text { Shock } \\
\text { Number }\end{array}$ & $\begin{array}{c}\text { Impedance } \\
(\Omega)\end{array}$ & $\begin{array}{l}\text { Delivered } \\
\text { Energy(J) }\end{array}$ & $\begin{array}{l}\text { Current } \\
\text { (A) }\end{array}$ \\
\hline 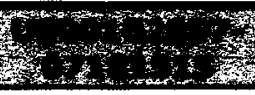 & 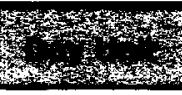 & (1) & 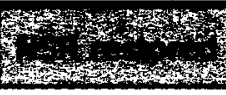 & 13y & & & 3 & 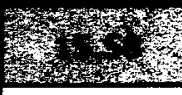 \\
\hline \multirow{2}{*}{$\begin{array}{c}\text { US00102857- } \\
07221427\end{array}$} & Day Unit & AFL & CV failed & Failure & 1 & 86 & 72 & 11.87 \\
\hline & & & 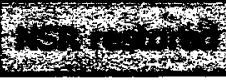 & & & & 30 & Wy, \\
\hline \multirow{2}{*}{$\begin{array}{c}\text { US00102857- } \\
07231440\end{array}$} & Day Unit & $\mathrm{AF}$ & CV failed & Failure & 1 & 84 & 101 & 16.92 \\
\hline & 1) & 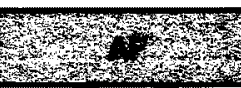 & 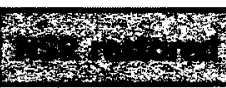 & 等 & & 7 & $x$ & 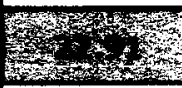 \\
\hline $\begin{array}{c}\text { US00102857- } \\
07241509\end{array}$ & Day Unit & AF & NSR restored & Success & 1 & 61 & 101 & 22.38 \\
\hline 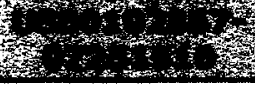 & & & 五 & (t) & & & & 作, \\
\hline \multirow{2}{*}{$\begin{array}{c}\text { US00102857- } \\
07291434\end{array}$} & Day Unit & AF & CV failed & Failure & 1 & 73 & 104 & 19.16 \\
\hline & $y^{2}$ & Writy & 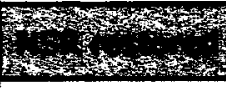 & Why & tysty & 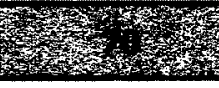 & (2) & Why \\
\hline $\begin{array}{c}\text { US00102857- } \\
07291606\end{array}$ & Day Unit & AF & NSR restored & Success & 1 & 56 & 149 & 29.25 \\
\hline
\end{tabular}














\section{REFERENCES}

[1] I. C. V. Gelder, H. J. Crijns, W. H. V. Gilst, R. Verwer, and K. I. Lie, "Prediction of uneventful cardioversion and maintenance of sinus rhythm from direct-current electrical cardioversion of chronic atrial fibrillation and flutter," The American Journal of Cardiology, vol. 68, no. 1, pp. 41-46, 1991.

[2] G. W. Dalzell, J. Anderson, and A. A. Adgey, "Factors determining success and energy requirements for cardioversion of atrial fibrillation," The Quarterly journal of medicine, vol. 78, no. 285, pp. 85-95, Jan. 1991.

[3] R. E. Kerber et al., "Energy, current, and success in defibrillation and cardioversion: clinical studies using an automated impedance-based method of energy adjustment," Circulation, vol. 77, no. 5, pp. 1038-46, May 1988.

[4] J. P. Heavens, M. J. Cleland, J. P. Maloney, and B. H. Rowe, "Effects of transthoracic impedance and peak current flow on defibrillation success in a prehospital setting," Annals of emergency medicine, vol. 32, no. 2, pp. 191-9, Aug. 1998.

[5] C. F. Babbs, W. A. Tacker, J. F. VanVleet, J. D. Bourland, and L. A. Geddes, "Therapeutic indices for transchest defibrillator shocks: Effective, damaging, and lethal electrical doses," American Heart Journal, vol. 99, no. 6, pp. 734-738, 1980.

[6] L. Resnekov and L. Mcdonald, "Complications in 220 Patients with Cardiac Dysrhythmias Treated by Phased Direct Current Shock, and Indications for Electroconversion," British Heart Journal, vol. 29, pp. 926-936, 1967.

[7] C. J. Chen and G. B. F. Guo, "External Cardioversion in Patients With Persistent Atrial Fibrillation," Japenese Heart Journal, vol. 44, no. 6, pp. 921-932, 2003.

[8] J. L. Jones and R. E. Jones, "Improved defibrillator waveform safety factor with biphasic waveforms.," The American journal of physiology, vol. 245, no. 1, pp. H60-5, Jul. 1983.

[9] Philips, "HeartStart MRx," 2010 [Service Manual].

[10] D. Keane, E. Boyd, D. Anderson, A. Robles, P. Deverall, R. Morris, G. Jackson, and E. Sowton, "Comparison of biphasic and monophasic waveforms in epicardial atrial defibrillation," Journal of the American College of Cardiology, vol. 24, no. 1, pp. 171-176, 1994.

[11] R. D. White, T. H. Blackwell, J. K. Russell, D. E. Snyder, and D. B. Jorgenson, "Transthoracic impedance does not affect defibrillation, resuscitation or survival in patients with out-of-hospital cardiac arrest treated with a non-escalating biphasic waveform defibrillator.," Resuscitation, vol. 64, no. 1, pp. 63-9, Jan. 2005.

[12] R. G. Walker, S. B. Melnick, F. W. Chapman, G. P. Walcott, P. W. Schmitt, and R. E. Ideker, "Comparison of six clinically used external defibrillators in swine,"

Resuscitation, vol. 57, no. 1, pp. 73-83, 2003. 
[13] G. W. Dalzell and A. A. Adgey, "Determinants of successful transthoracic defibrillation and outcome in ventricular fibrillation.," Heart, vol. 65, no. 6, pp. 311-316, Jun. 1991.

[14] A. C. Guyton and J. E. Hall, Textbook of Medical Physiology, 11th ed. Philadelphia: Saunders, 1981.

[15] P. Crockett, B. Droppert, and S. Higgins, Defibrillation: What you should know. Redmond,Washington: Physio-Control Corporation, 1996.

[16] D. P. Zipes, J. Fischer, R. M. King, A. deB. Nicoll, and W. W. Jolly, "Termination of ventricular fibrillation in dogs by depolarizing a critical amount of myocardium," The American Journal of Cardiology, vol. 36, no. 1, pp. 37-44, 1975.

[17] W. E. Garrey, "The nature of fibrillatory contractions of the heart-it's relation to tissue mass and form," American Journal of Physiology, vol. 33, pp. 397-414, 1914.

[18] P. Chen, P. D. Wolf, S. D. Melnick, N. D. Danieley, W. M. Smith, and R. E. Ideker, "Comparison of Activation During Ventricular Fibrillation and Following Unsuccessful Defibrillation Shocks in Open-Chest Dogs," Circulation Research, pp. 1544-1560, 1990.

[19] G. P. Walcott, C. R. Killingsworth, and R. E. Ideker, "Do clinically relevant transthoracic defibrillation energies cause myocardial damage and dysfunction?," Resuscitation, vol. 59, no. 1, pp. 59-70, Oct. 2003.

[20] J. L. Jones and O. H. Tovar, "The mechanism of defibrillation and cardioversion," Proceedings of the IEEE, vol. 84, no. 3, pp. 392-403, Mar. 1996.

[21] S. M. Dillon, "Optical Recordings in the Rabbit Heart Show That Defibrillation Strength Shocks Prolong the Duration of Depolarization and the Refractory Period," Circulation Research, vol. 69, pp. 842-856, 1991.

[22] J. P. Wikswo, S. Lin, and R. A. Abbas, "Virtual Electrodes in Cardiac Tissue: A Common Mechanism for Anodal and Cathodal Stimulation," Biophysical Journal, vol. 69, pp. 2195-2210, Dec. 1995.

[23] I. R. Efimov, Y. Cheng, D. R. V. Wagoner, T. Mazgalev, and J. Tchou, "Virtual Electrode - Induced Phase Singularity A Basic Mechanism of Defibrillation Failure," Circulation Research, vol.82, pp. 918-925, 1998.

[24] B. Lown, R. Amarasingham, and J. Neuman, "New method for terminating cardiac arrhythmias. Use of synchronized capacitor discharge.," Jama The Journal of The American Medical Association, vol. 182, no. 17, pp. 548-555, 1962.

[25] M. Block and G. Breithardt, "Optimizing Defibrillation Through Improved Waveforms," Pacing and Clinical Electrophysiology, vol. 18, no. March, pp. 526-539, 1995.

[26] H. L. Greene et al., "Comaprison of Monophasic and Biphasic Defibrillating Pulse Waveforms for Transthoracic Cardioversion," American Journal of Cardiology, vol. 75, pp. 1135-1139, 1995. 
[27] K. M. Kavanagh, H. J. Duff, R. Clark, K. V. Robinson, W. R. Giles, And D. G. Wyse, "Monophasic Versus Biphasic Cardiac Stimulation: Mechanism of Decreased Energy Requirements," Pacing and Clinical Electrophysiology, vol. 13, no. 10, pp. 1268-1276, 1990.

[28] S. Mittal et al., "Comparison of a novel rectilinear biphasic waveform with a damped sine wave monophasic waveform for transthoracic ventricular defibrillation. ZOLL Investigators.," Journal of the American College of Cardiology, vol. 34, no. 5, pp. 1595-601, Nov. 1999.

[29] M. Scholten, T. Torok, P. Klootwijk, and L. Jordaens, "Comparison of monophasic and biphasic shocks for transthoracic cardioversion of atrial fibrillation," Heart (British Cardiac Society), vol. 89, no. 9, pp. 1032-4, Sep. 2003.

[30] R. W. Rho and R. L. Page, "Biphasic versus monophasic shock waveform for conversion of atrial fibrillation," Springer, vol. 7, pp. 290-291, 2003.

[31] R. L. Page et al., "Biphasic Versus Monophasic Shock Waveform for Conversion of Atrial Fibrillation," American Journal of Cardiology, vol. 39, pp. 1956-1963, 2002.

[32] S. Sun, K. Klouche, W. Tang, and M. H. Weil, "The effects of biphasic and conventional monophasic defibrillation on postresuscitation myocardial function.," Journal of the American College of Cardiology, vol. 37, no. 6, pp. 1753-4, May 2001.

[33] V. S. Kawabata, C. Vianna, M. Moretti, M. Gonzalez, J. Ferreira, S. Timerman, and L. Cesar, "Monophasic versus biphasic waveform shocks for atrial fibrillation cardioversion in patients with concomitant amiodarone therapy," Europace, vol. 9, no. 2, pp. 143-146, Feb. 2007.

[34] G. P. Walcott, R.G. Walker, A. W. Cates, W. Krassowska, W. M. Smith, R. Ideker, "Choosing the Optimal Monophasic and Biphasic Waveforms for Ventricular Defibrillation," Journal of Cardiovascular Electrophysiology, vol.6, pp. 737-750, Sep. 1995.

[35] M. Marinsek, G. L. Larkin, P. Zohar, M. Bervar, M. Pekolj-Bicanic, F. S. Mocnik, M. Noc, and M. Podbregar, "Efficacy and impact of monophasic versus biphasic countershocks for transthoracic cardioversion of persistent atrial fibrillation," The American Journal of Cardiology, vol. 92, no. 8, pp. 988-991, 2003.

[36] J. L. Jones, R. E. Jones, and G. Balasky, "Improved cardiac cell excitation with symmetrical biphasic defibrillator waveforms.," The American journal of physiology, vol. 253, no. 6 Pt 2, pp. H1418-24, Dec. 1987.

[37] S. J. Walsh, B. M. Glover, and A. A. J. Adgey, "The Role of Biphasic Shocks for Transthoracic Cardioversion of Atrial Fibrillation," Indian Pacing and Electrophysiology Journal, vol. 5, no. 4, pp. 289-295, Oct. 2005.

[38] S. Mittal et al., "Transthoracic Cardioversion of Atrial Fibrillation:Comparison of Rectilinear Biphasic Versus Damped Sine Wave Monophasic Shocks," Circulation, vol. 101, pp. 1282-1287, 2000. 
[39] A. S. L. Tang, S. Yabe, J. M. Wharton, M. Dolker, W. M. Smith, and R. E. Ideker, "Ventricular defibrillation using biphasic waveforms: The importance of phasic duration," Journal of the American College of Cardiology, vol. 13, no. 1, pp. 207-214, 1989.

[40] R. E. Hillsley, R. G. Walker, D. K. Swanson, D. L. Rollins, P. D. Wolf, W. M. Smith, and R. E. Ideker, "Is the Second Phase of a Biphasic Defibrillation Waveform the Defibrillating Phase?," PACE, vol. 16, pp. 1401-1412, July 1993.

[41] J. F. Swartz, J. L. Jones, R. E. Jones, and R. Fletcher, "Conditioning Prepulse of Biphasic Defibrillator Waveforms Enhances Refractoriness to Fibrillation Wavefronts," Circulation Research, pp. 438-449, 1991.

[42] J. L. Jones, R. E. Jones, and G. Balasky, "Microlesion formation in myocardial cells by high-intensity electric field stimulation.," The American journal of physiology, vol. 253, no. 2, pp. H480-6, Aug. 1987.

[43] W. Lammers, M. Schalij, C. Kirchhof, and A. Allessie, "Quantification of spatial inhomogeneity in conduction and initiation of reentrant atrial arrhythmias," American Journal of Cardiology, vol. 259, pp. 1254-1263, 1990.

[44] B. Weight, C. Size, and S. Shocks, "Transthoracic Resistance in Human Defibrillation," Circulation, vol. 63, pp. 676-682, 1981.

[45] C. D. Deakin, D. M. Sado, G. W. Petley, and F. Clewlow, "Determining the optimal paddle force for external defibrillation.," The American journal of cardiology, vol. 90, no. 7, pp. 812-3, Oct. 2002.

[46] N. A. Paradis, H. R. Halperin, K. B. Kern, V. Wenzel, and D. A. Chmaberlain, Cardiac Arrest- The Science and Practice of Resuscitation Medicine, Second. Cambridge: Cambridge University Press, 2007.

[47] G. A. Ewy, D. Hellman, S. McClung, and D. Taren, "Influence of ventilation phase on transthoracic impedance and defibrillation effectivess," Critical Care Medicine, vol. 8, pp. $164-166,1980$.

[48] G. A. Ewy, "The Optimal Technique for Electrical Cardioversion of Atrial Fibrillation," Clinical Cardiology, vol. 17, no. 2, pp. 79-84, 1994.

[49] M. R. Caterine, D. M. Yoerger, K. T. Spencer, S. G. Miller, and R. E. Kerber, "Effect of electrode position and gel-application technique on predicted transcardiac current during transthoracic defibrillation.," Annals of emergency medicine, vol. 29, no. 5, pp. 588-95, May 1997.

[50] G. L. Botto, A. Politi, W. Bonini, T. Brovoni, and R. Bonatti, "External cardioversion of atrial fibrillation : role of paddle position on technical efficacy and energy requirements," Heart, vol. 82, pp. 726-730, 1999.

[51] M. a Camacho, J. L. Lehr, and S. R. Eisenberg, "A three-dimensional finite element model of human transthoracic defibrillation: paddle placement and size.," IEEE transactions on bio-medical engineering, vol. 42, no. 6, pp. 572-8, Jun. 1995. 
[52] L. C. Hunt and A. L. de Jongh Curry, "Finite element computer modeling of transthoracic atrial defibrillation," The 26th Annual International Conference of the IEEE Engineering in Medicine and Biology Society, vol. 4, pp. 3964-3967, 2004.

[53] S. J. Walsh et al., "Impedance compensated biphasic waveforms for transthoracic cardioversion of atrial fibrillation: a multi-centre comparison of antero-apical and antero-posterior pad positions.," European heart journal, vol. 26, no. 13, pp. 1298-302, Jul. 2005.

[54] J. L. Jones, D. E. Snyder, and C. Morgan, "Predictions from misleading pig model are potentially harmful to humans," Resuscitation, vol. 59, no. 3, pp. 365-367, 2003.

[55] M. Kotikanyadanam, S. Goktepe, and E. Kuhl, "Computational modeling of electrocardiograms : A finite element approach toward cardiac excitation," Int. J. Numer. Meth. Biomed. Engng, vol 26, pp. 524-533, 2010.

[56] N. G. Sepulveda, J. P. Wikswo, and D. S. Echt, "Finite element analysis of cardiac defibrillation current distributions.," IEEE transactions on bio-medical engineering, vol. 37, no. 4, pp. 354-65, Apr. 1990.

[57] W. J. Karlon, S. R. Eisenberg, and J. L. Lehr, "Effects of paddle placement and size on defibrillation current distribution: a three-dimensional finite element model.," IEEE transactions on bio-medical engineering, vol. 40, no. 3, pp. 246-55, Mar. 1993.

[58] T. F. Kinst, M. O. Sweeney, J. L. Lehr, and S. R. Eisenberg, "Simulated internal defibrillation in humans using an anatomically realistic three-dimensional finite element model of the thorax.," Journal of cardiovascular electrophysiology, vol. 8, no. 5, pp. 537-47, May 1997.

[59] J. K. Triedman, M. Jolley, J. Stinstra, D. H. Brooks, and R. MacLeod, "Predictive modeling of defibrillation using hexahedral and tetrahedral finite element models: recent advances," Journal of Electrocardiology, vol. 41, no. 6, pp. 483-486, Nov. 2008.

[60] J. C. Eason, C. M. Clark, P. D. Wolf, J. a. Schmidt, and R. E. Ideker, "An accurate finite element model of the heart for calculating the electric field due to defibrillation," Proceedings of Computers in Cardiology Conference, pp. 57-59, 1993.

[61] D. Panescu, J. G. Webster, W. J. Tompkins, and R. a. Stratbucker, "Optimization of cardiac defibrillation by three-dimensional finite element modeling of the human thorax," IEEE Transactions on Biomedical Engineering, vol. 42, no. 2, pp. 185-192, 1995.

[62] P. H. Schimpf, G. Johnson, D. B. Jorgenson, D. R. Haynor, G. H. Bardy, and Y. Kim, "Effects of electrode interface impedance on finite element models of transvenous defibrillation.," Medical \& biological engineering \& computing, vol. 33, no. 5, pp. 713-9, Sep. 1995.

[63] K. T. Ng, S. A. Hutchinson, and S. Gao, "Numerical analysis of electrical defibrillation. The parallel approach.," Journal of electrocardiology, vol. 28 Suppl, pp. 15-20, Jan. 1995. 
[64] D. B. Jorgenson, D. R. Haynor, G. H. Bardy, and Y. Kim, "Computational studies of transthoracic and transvenous defibrillation in a detailed 3-D human thorax model," IEEE transactions on bio-medical engineering, vol. 42, no. 2, pp. 172-84, Feb. 1995.

[65] A L. de Jongh, E. G. Entcheva, J. A. Replogle, R. S. Booker, B. H. Kenknight, and F. J. Claydon, "Defibrillation efficacy of different electrode placements in a human thorax model," Pacing and clinical electrophysiology: PACE, vol. 22, no. 1 Pt 2, pp. 152-7, Jan. 1999.

[66] E. Vigmond, F. Vadakkumpadan, V. Gurev, H. Arevalo, M. Deo, G. Plank, and N. Trayanova, "Towards predictive modelling of the electrophysiology of the heart," Experimental physiology, vol. 94, no. 5, pp. 563-77, May 2009.

[67] Y. Zhang et al., "Triphasic waveforms are superior to biphasic waveforms for transthoracic defibrillation," Journal of the American College of Cardiology, vol. 42, no. 3, pp. 568-575, Aug. 2003.

[68] J. L. Jones and R. E. Jones, "Improved safety factor for triphasic defibrillator waveforms," Circulation Research, vol. 64, no. 6, pp. 1172-1177, 1989.

[69] S. Rosenheck, S. Gorni, I. Katz, A. Rabin, U. Shpoliansky, M. Mandelbaum, and A. T. Weiss, "Modified alternating current defibrillation: a new defibrillation technique," Europace, vol. 11, no. 2, pp. 239-244, 2009.

[70] B. E. Gliner, T. E. Lyster, S. M. Dillion, and G. H. Bardy, "Transthoracic Defibrillation of Swine With Monophasic and Biphasic Waveforms," Circulation, vol. 92, no. 6, pp. 1634-1643, Sep. 1995.

[71] S. A. Feeser, A. S. Tang, K. M. Kavanagh, D. L. Rollins, W. M. Smith, P. D. Wolf, R. E. Ideker, "Strength-duration and probability of success curves for defibrillation with biphasic waveforms," Circulation, vol. 82, no. 6, pp. 2128-2141, 1990.

[72] L. Jones, B. Milne, J. L, and K. B. Milne, "Dysfunction and safety factor strengthduration curves for biphasic defibrillator waveforms," American Journal of Physiology, vol. 266, pp. H263-71, 1994.

[73] G. Frithz and H. Aberg, "Direct current conversion of atrial flutter," Acta Medica Scandinavica, vol. 187, no. 4, pp. 271-274, 1970.

[74] A. Castellanos, A. Gosselin, and E. J. Fonseca, "Evaluation of Countershock Treatment Of Atrial Flutter with Special Reference To Arrhythmias Related To This Procedure," Archives of Internal Medicine, vol. 115, pp. 426-433, 1965.

[75] G. K. Mah, "Cardioversion in the treatment of atrial flutter," Singapore Medical Journal, vol. 10, no. 3, pp. 164-169, 1969.

[76] K. J. Tucker and C. Wilson, "A comparison of transoesophageal atrial pacing and direct current cardioversion for the termination of atrial flutter: a prospective, randomised clinical trial.," British Heart Journal, vol. 69, no. 6, pp. 530-535, 1993. 
[77] "Philips SMART Biphasic therapy" [Online]. Accessed: 27-07-2012. Available: http://incenter.medical.philips.com/doclib/enc/fetch/2000/4504/577242/577243/577 245/577817/577869/SMART_Biphasic_Application_Note_\%28ENG\%29.pdf\%3fnodeid $\% 3 d 578140 \% 26$ vernum\%3d3

[78] B. Graham, "Enhancements in Electrical Impedance Tomography(EIT) Image Reconstruction for 3D Lung Imaging," University of Ottawa, 2007.

[79] J. Schöberl, "NETGEN - An advancing front 2D/3D-mesh generator based on abstract rules," Computing and Visualization in Science, vol. 1, pp. 41-52, 1997.

[80] A. Adler and W. R. B. Lionheart, "Uses and abuses of EIDORS: an extensible software base for EIT.," Physiological Measurement, vol. 27, no. 5, pp. S25-S42, 2006.

[81] K. R. Foster, "Dielectric Properties of tissues," in The Biomedical Engineering Handbook, 2nd ed., J. D. Bronzino, Ed. CRC Press Inc., 1999.

[82] J. Wtorek, "Relations between components of impedance cardiogram analyzed by means of Finite Element Model and Sensitivity Theorem," Annals of Biomedical Engineering, vol. 28, no. 11, pp. 1352-1361, 2000.

[83] F. Lateef, S. H. Lim, V. Anantharaman, and C. S. Lim, "Chest impedance: characteristics of local patients," Singapore Medical Journal, vol. 41, no. 7, pp. 331-334, 2000.

[84] Philips Medical System, "Biphasic Waveform Technology," 2004, [Online] Accessed : 27-07-2012, Available:

http://www.touchbriefings.com/pdf/1102/Philips_techproof.pdf.

[85] D. Konakanchi and A. L. de Jongh Curry, "Simultaneous double external DC shock techniques for atrial fibrillation: a simulation study," Annual International Conference of the IEEE Engineering in Medicine and Biology Society. IEEE Engineering in Medicine and Biology Society Conference, vol. 2008, pp. 1745-8, Jan. 2008.

[86] R. E. Kerber, S. R. Jensen, J. Grayzel, J. Kennedy, and R. Hoyt, "Elective Cardioversion: Influence of Paddle-Electrode Location and Size on Success Rates and Energy Requirements," New England Journal of Medicine, vol. 305, no. 12, pp. 658-662, 1981. 